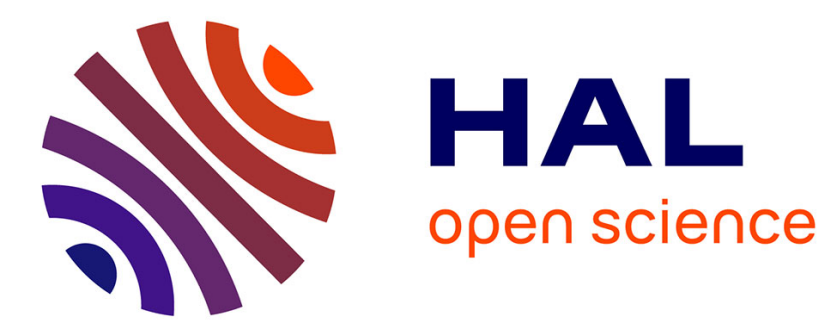

\title{
Mean exit time for the overdamped Langevin process: the case with critical points on the boundary \\ Boris Nectoux
}

\section{To cite this version:}

Boris Nectoux. Mean exit time for the overdamped Langevin process: the case with critical points on the boundary. 2020. hal-02196801v2

\section{HAL Id: hal-02196801 \\ https://hal.science/hal-02196801v2}

Preprint submitted on 8 Oct 2020 (v2), last revised 7 Apr 2022 (v4)

HAL is a multi-disciplinary open access archive for the deposit and dissemination of scientific research documents, whether they are published or not. The documents may come from teaching and research institutions in France or abroad, or from public or private research centers.
L'archive ouverte pluridisciplinaire HAL, est destinée au dépôt et à la diffusion de documents scientifiques de niveau recherche, publiés ou non, émanant des établissements d'enseignement et de recherche français ou étrangers, des laboratoires publics ou privés. 


\title{
Mean exit time for the overdamped Langevin process: the case with critical points on the boundary
}

\begin{abstract}
Boris Nectoux *
Abstract

Let $\left(X_{t}\right)_{t \geq 0}$ be the overdamped Langevin process on $\mathbb{R}^{d}$, i.e. the solution of the stochastic differential equation

$$
d X_{t}=-\nabla f\left(X_{t}\right) d t+\sqrt{h} d B_{t} .
$$

Let $\Omega \subset \mathbb{R}^{d}$ be a bounded domain. In this work, when $X_{0}=x \in \Omega$, we derive new sharp asymptotic equivalents (with optimal error terms) in the limit $h \rightarrow 0$ of the mean exit time from $\Omega$ of the process $\left(X_{t}\right)_{t \geq 0}$ (which is the solution of $\left(-\frac{h}{2} \Delta+\right.$ $\nabla f \cdot \nabla) w=1$ in $\Omega$ and $w=0$ on $\partial \Omega$ ), when the function $f: \bar{\Omega} \rightarrow \mathbb{R}$ has critical points on $\partial \Omega$. Such a setting is the one considered in many cases in molecular dynamics simulations. This problem has been extensively studied in the literature but such a setting has never been treated. The proof, mainly based on techniques from partial differential equations, uses recent spectral results from 30] and its starting point is a formula from the potential theory. We also provide new sharp leveling results on the mean exit time from $\Omega$.
\end{abstract}

Key words: singular perturbation, asymptotic analysis, overdamped Langevin process, mean exit time, metastability, Eyring-Kramers formulas.

AMS classification (2010): 31C15, 60J60, 35P15.

\section{Purpose of this work and main result}

\subsection{Setting and purpose of this work}

Let us consider a function $f: \mathbb{R}^{d} \rightarrow \mathbb{R}$. A prototypical process used to model the evolution of a statistical system in a constant temperature environment is the so-called overdamped Langevin process, i.e. the solution $\left(X_{t}\right)_{t \geq 0}$ of the stochastic differential equation

$$
d X_{t}=-\nabla f\left(X_{t}\right) d t+\sqrt{h} d B_{t},
$$

where $h>0$ is the temperature of the medium and $\left(B_{t}\right)_{t \geq 0}$ is a $d$-dimensional standard Brownian motion. For an open subset $\Omega$ of $\mathbb{R}^{d}$, one denotes by

$$
\tau_{\Omega^{c}}=\inf \left\{t \geq 0, X_{t} \notin \Omega\right\}
$$

the first time the process $(1)$ enters in $\Omega^{c}:=\mathbb{R}^{d} \backslash \Omega$ (or equivalently the first exit time from $\Omega$ ). The aim of this paper is to prove sharp asymptotic estimates when $h \rightarrow 0$, with optimal error terms, on the mean exit time $\mathbb{E}\left[\tau_{\Omega^{c}}\right]$ from a domain $\Omega \subset \mathbb{R}^{d}$ of the process (1) when $f$ has critical point on $\partial \Omega$. The resulting equivalents are usually called Eyring-Kramers type formulas, see 20] for a review on this topic. Such a setting is motivated by the fact that in many molecular

\footnotetext{
${ }^{*}$ Institut für Analysis und Scientific Computing, TU Wien, Wiedner Hauptstr. 8, 1040 Wien, Austria. E-mail: boris.nectoux@asc.tuwien.ac.at
} 
dynamics simulations (where several algorithms have been designed to accelerate the sampling of the exit event $\left(\tau_{\Omega^{c}}, X_{\tau_{\Omega^{c}}}\right)$ ), the domain $\Omega$ is defined as a basin of attraction for the $-\nabla f$ dynamics (see (4) below) of a local minimum of $f$ in $\mathbb{R}^{d}$ so that $f$ has critical points on $\partial \Omega$. To analyse the mathematical foundations of these algorithms, it is necessary to study the precise asymptotic behavior of the law of $\left(\tau_{\Omega^{c}}, X_{\tau_{\Omega^{c}}}\right)$ as $h \rightarrow 0$ (see for instance $[13,31,34,48,51,52$ and references therein). Moreover, our techniques also allow us to consider the case when $f: \bar{\Omega} \rightarrow \mathbb{R}$ has a degenerate principal barrier in $\Omega$, i.e. for some $m>1$,

$$
\lim _{h \rightarrow 0} h \ln \lambda_{1, h}=\lim _{h \rightarrow 0} h \ln \lambda_{2, h}=\ldots=\lim _{h \rightarrow 0} h \ln \lambda_{m, h},
$$

where $\lambda_{1, h}<\lambda_{2, h} \leq \ldots \leq \lambda_{m, h}$ are the $m$ smallest eigenvalues of the Dirichlet realization of infinitesimal generator of the diffusion (1) in $\Omega$ (see the next section for the definition of this operator). Despite the attention this topic has received these past few decades, to the best of our knowledge, such settings have not been treated yet (see Section 1.5 below). Furthermore, dealing with a degenerate principal barrier introduces some subtleties if one wants to study the asymptotic behavior of $\mathbb{E}\left[\tau_{\Omega^{c}}\right]$ via a spectral approach. This is due to the fact that the $m$ smallest eigenvalues $\lambda_{1, h}, \ldots, \lambda_{m, h}$ can be asymptotically indistinguishable when $h \rightarrow 0$, as explained in [29]. To overcome these difficulties, we introduce local Dirichlet problems (see Section 2.5 below) and use recent spectral results from 30 together with formulas from the potential theory and estimates from the large deviations theory.

\subsection{Notation and definitions}

In all this work, $\Omega$ is a $\mathcal{C}^{\infty}$ bounded open and connected subset of $\mathbb{R}^{d}$, and the function $f: \bar{\Omega} \rightarrow \mathbb{R}$ is $\mathcal{C}^{\infty}$. Throughout this work, we will use the following notation: for $a \in \mathbb{R}$,

$$
\{f<a\}=\{x \in \bar{\Omega}, f(x)<a\} \text { and }\{f=a\}=\{x \in \bar{\Omega}, f(x)=a\} .
$$

Moroever, $\mathrm{B}_{r}\left(x_{*}\right)$ will denote the closed ball centred at $x_{*}$ of radius $r>0$ in $\mathbb{R}^{d}$. Finally, $\mathbb{E}_{x}$ (resp. $\mathbb{P}_{x}$ ) will denote the expectation (resp. the probability) when $X_{0}=x \in \mathbb{R}^{d}$.

Dirichlet realization of $-\frac{h}{2} \Delta+\nabla f \cdot \nabla$ in $\Omega$. Let us denote by $\mathcal{L}_{f, h}=-\frac{h}{2} \Delta+\nabla f \cdot \nabla$ the infinitesimal generator of the process (1). The operator $\mathcal{L}_{f, h}$ on $L^{2}\left(\Omega, e^{-\frac{2}{h} f} d x\right)$ with domain $\left\{w \in H^{2}\left(\Omega, e^{-\frac{2}{h} f} d x\right), w=0\right.$ on $\left.\partial \Omega\right\}$ is denoted by $\mathcal{L}_{f, h}^{\text {Dir }}$ (where the notation Dir stands for the fact that we consider $\mathcal{L}_{f, h}$ with Dirichlet boundary conditions on $\partial \Omega$ ). The operator $\mathcal{L}_{f, h}^{\text {Dir }}$ is the Friedrichs extension on $L^{2}\left(\Omega, e^{-\frac{2}{h} f} d x\right)$ of the closed quadratic form

$$
g \in H_{0}^{1}\left(\Omega, e^{-\frac{2}{h} f} d x\right) \mapsto \frac{2}{h} \int_{\Omega}|\nabla g|^{2} e^{-\frac{2}{h} f} .
$$

The operator $\mathcal{L}_{f, h}^{\text {Dir }}$ is a positive self adjoint operator on $L^{2}\left(\Omega, e^{-\frac{2}{h} f} d x\right)$ with compact resolvent. Its eigenvalues are denoted by

$$
\lambda_{1, h}<\lambda_{2, h} \leq \lambda_{3, h} \ldots
$$

where we recall that by standard results on elliptic operators, $\lambda_{1, h}$ is simple and any associated eigenfunction $u_{1, h}$ is $\mathcal{C}^{\infty}$ on $\bar{\Omega}$ and has a sign on $\Omega$. Finally let us recall that $\mathcal{L}_{f, h}$ is connected to the Witten Laplacian $\Delta_{f, h}=h^{2} \Delta_{H}+|\nabla f|^{2}-h \Delta f$ through the relation

$$
\mathcal{L}_{f, h}=\frac{1}{2 h} e^{\frac{1}{h} f} \Delta_{f, h} e^{-\frac{1}{h} f},
$$


which will allow us to transfer the results of [30] on the spectrum of the Dirichlet realization of $\Delta_{f, h}$ in $\Omega$ to $\mathcal{L}_{f, h}^{\text {Dir }}$.

Domain of attraction of a subset $\mathrm{M}$ of $\Omega$ for the $-\nabla f$ dynamics in $\Omega$. Let $x \in \Omega$ and denote by $\varphi_{t}(x)$ the solution to the ordinary differential equation

$$
\frac{d}{d t} \varphi_{t}(x)=-\nabla f\left(\varphi_{t}(x)\right) \text { with } \varphi_{0}(x)=x,
$$

on the interval $t \in\left[0, t_{x}\right]$, where $t_{x}=\inf \left\{t \geq 0, \varphi_{t}(x) \notin \Omega\right\}>0$. Let $x \in \Omega$ be such that $t_{x}=+\infty$. The $\omega$-limit set of $x$, denoted by $\omega(x)$, is defined by

$$
\omega(x)=\left\{y \in \bar{\Omega}, \exists\left(s_{n}\right)_{n \in \mathbb{N}} \in\left(\mathbb{R}_{+}\right)^{\mathbb{N}}, \lim _{n \rightarrow \infty} s_{n}=+\infty, \lim _{n \rightarrow \infty} \varphi_{s_{n}}(x)=y\right\} .
$$

Let us recall that the $\omega$-limit set $\omega(x)$ is included in the set of the critical points of $f$ in $\bar{\Omega}$. Moreover, if $f$ has a finite number of critical points in $\bar{\Omega}$, there exists $y \in \bar{\Omega}$ such that $\omega(x)=$ $\{y\} \subset\{z \in \bar{\Omega},|\nabla f(z)|=0\}$. The domain of attraction of $\mathrm{M}$ is then defined by

$$
\mathcal{A}_{\Omega}(\mathrm{M})=\left\{x \in \Omega, t_{x}=+\infty \text { and } \omega(x) \subset \mathrm{M}\right\} .
$$

Principal wells of $f$ in $\Omega$. In all this work, we will assume that the function $f: \bar{\Omega} \rightarrow \mathbb{R}$ a Morse function, i.e. that all its critical points are non degenerate. In particular, $f$ has finite number of critical points in $\bar{\Omega}$. Moreover, we shall say that $z \in \bar{\Omega}$ is a saddle point of $f$ if it is a critical point of index 1 of $f$. Let us finally recall the definition of the principal wells of $f$ in $\Omega$ which are defined in $\left[30\right.$, Section 3.1] when $f: \bar{\Omega} \rightarrow \mathbb{R}$ is a $\mathcal{C}^{\infty}$ Morse function (see also [14, Section 2.1]). For all local minimum $x$ of $f$ in $\Omega$ and $\lambda>f(x)$, we denote by $\mathrm{C}(\lambda, x)$ the connected component of $\{f<\lambda\}$ in $\bar{\Omega}$ containing $x$. Moroever, one defines

$$
\lambda(x):=\sup \{\lambda>f(x) \text { s.t. } \mathrm{C}(\lambda, x) \cap \partial \Omega=\emptyset\} \quad \text { and } \quad \mathrm{C}(x):=\mathrm{C}(\lambda(x), x) .
$$

The set of principal wells of $f$ in $\Omega$ is the set

$$
\mathcal{C}:=\{\mathrm{C}(x), x \text { is a local minimum of } f \text { in } \Omega\} .
$$

From [30, Proposition 7], each $\mathcal{C} \in \mathcal{C}$ is included in $\Omega$ and the elements of $\mathcal{C}$ are two by two disjoint. Notice finally that for each $\mathrm{C} \in \mathcal{C}$, it holds $\mathrm{C} \subset \mathcal{A}_{\Omega}(\mathrm{C})$ since $\mathrm{C}$ is stable for the dynamics (4) (because $f$ is non increasing along the curves defined by (4)).

\subsection{Assumptions on the function $f$}

Let us now introduce the basic assumption of this work.

Assumption (H1). The function $f: \bar{\Omega} \rightarrow \mathbb{R}$ is a $\mathcal{C}^{\infty}$ Morse function and the set $\left\{f<\min _{\partial \Omega} f\right\}$ is non empty and contains all the local minima of $f$ in $\Omega$. Furthermore, denoting by $\mathrm{C}_{1}, \ldots, \mathrm{C}_{\mathrm{N}}$ the connected components of $\left\{f<\min _{\partial \Omega} f\right\}$, it holds for all $j \in\{1, \ldots, N\}$ :

$$
\partial \mathrm{C}_{j} \cap \partial \Omega \neq \emptyset \text { and } \overline{\mathrm{C}_{j}} \text { is a connected component of }\left\{f \leq \min _{\partial \Omega} f\right\} .
$$

A schematic representation of $C_{1}, \ldots, C_{N}$ is given in Figure 1 when $N=2$ (see also Figure 7 below). Let us now comment the assumption (H1). Notice that under (H1), one has:

$$
\min _{\bar{\Omega}} f<\min _{\partial \Omega} f \text { and for all } j \in\{1, \ldots, \mathrm{N}\}, \mathrm{C}_{j} \subset \Omega .
$$

The following lemma will allow us to use the results of [30]. 
Lemma 1. When (H1) holds, according to the terminology introduced in [30] (see also (7)), the function $f$ has exactly $\mathrm{N}$ principal wells in $\Omega$ which are $\mathrm{C}_{1}, \ldots, \mathrm{C}_{\mathrm{N}}$.

Proof. Under (H1), it follows that for all local minima $x$ of $f$ in $\Omega, \lambda(x)=\min _{\partial \Omega} f$ and $\mathrm{C}(x)=\mathrm{C}_{j}$ where $j \in\{1, \ldots, \mathrm{N}\}$ is such that $x \in \mathrm{C}_{j}$. Thus, it holds $\mathcal{C}=\left\{\mathrm{C}_{1}, \ldots, \mathrm{C}_{\mathrm{N}}\right\}$.

From Lemma 1 and [30, Proposition 11], for $z \in \partial \mathrm{C}_{j} \cap \partial \Omega$ (notice that $z \in \arg \min _{\partial \Omega} f$ ) one has:

a. If $|\nabla f(z)|=0$, then, $z$ is a saddle point of $f$.

$b$. If $|\nabla f(z)| \neq 0$, then $\partial_{\mathrm{n}} f(z)>0$, where $\mathrm{n}$ is the unit outward normal vector to $\Omega$.

For $j \in\{1, \ldots, \mathbf{N}\}$, the assumption that $\partial \mathrm{C}_{j} \cap \partial \Omega \neq \emptyset$ ensures that the exit event from $\Omega$ when $X_{0}=x \in \mathcal{A}_{\Omega}\left(\mathrm{C}_{j}\right)$ is associated with a point $z \in \partial \mathrm{C}_{j} \cap \partial \Omega$ when $h \rightarrow 0$ (see indeed the prefactor appearing in the asymptotic equivalents of $\mathbb{E}_{x}\left[\tau_{\Omega^{c}}\right]$ in Theorem 1 below).

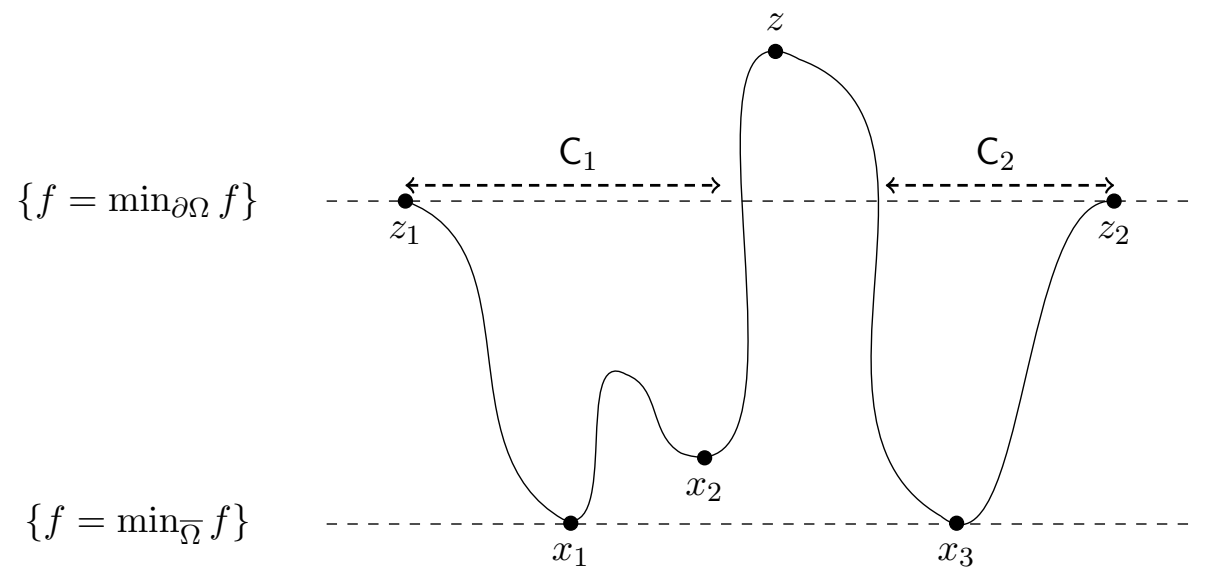

Figure 1: A one dimensional case when (H1) is satisfied with $\mathrm{N}_{0}=\mathrm{N}=2$ (notice that (H2) is always satisfied in dimension one), see (8). Here, the set of local minima of $f$ is $\left\{x_{1}, x_{2}, x_{3}\right\}$ with $\arg \min _{\bar{\Omega}} f=\left\{x_{1}, x_{3}\right\}$. The points $z_{1}$ and $z_{2}$ satisfy $z_{1} \in \partial \mathrm{C}_{1} \cap \partial \Omega$, $f^{\prime}\left(z_{1}\right)=0$, and $f^{\prime}\left(z_{2}\right)=0$. Moreover, $\mathcal{A}_{\Omega}\left(\mathrm{C}_{1}\right)=\left(z_{1}, z\right), \mathcal{A}_{\Omega}\left(\mathrm{C}_{2}\right)=\left(z, z_{2}\right)$.

When (H1) is satisfied, we denote by $\mathrm{N}_{0}$ the number of $\mathrm{C}_{j}$ 's which contain a point in $\arg \min _{\bar{\Omega}} f$, i.e.

$$
\mathrm{N}_{0}:=\operatorname{Card}\left(j \in\{1, \ldots, \mathrm{N}\} \text { s.t. } \underset{\bar{\Omega}}{\arg \min } f \cap \mathrm{C}_{j} \neq \emptyset\right) .
$$

In all this work, we will assume that $\left\{\mathrm{C}_{1}, \ldots, \mathrm{C}_{\mathrm{N}}\right\}$ are ordered such that for all $j \in\left\{1, \ldots, \mathrm{N}_{0}\right\}$, $\arg \min _{\bar{\Omega}} f \cap C_{j} \neq \emptyset$. Let us now introduce the following and last assumption on the function $f$.

Assumption (H2). The function $f: \bar{\Omega} \rightarrow \mathbb{R}$ satisfies (H1) and for all $j \in\{1, \ldots, \mathrm{N}\}$ and $z \in \partial \mathbf{C}_{j} \cap \partial \Omega$,

a. If $|\nabla f(z)| \neq 0, z$ is a nondegenerate global minimum of $\left.f\right|_{\partial \Omega}$.

b. If $|\nabla f(z)|=0$, the outward normal $\mathrm{n}_{\Omega}(z)$ to $\Omega$ at $z$ is an eigenvector associated with the negative eigenvalue of Hess $f(z)$. 
When (H1) and (H2) hold, from [30, Theorem 2], Lemma 1, and (3), there exists $C>0$, for all $j \in\left\{1, \ldots, N_{0}\right\}$, there exits $\beta_{j} \in\{0,-1 / 2\}$ such that, for $h$ small enough:

$$
C^{-1} h^{\beta_{j}} e^{-\frac{2}{h}\left(\min _{\partial \Omega} f-\min _{\bar{\Omega}} f\right)} \leq \lambda_{j, h} \leq C h^{\beta_{j}} e^{-\frac{2}{h}\left(\min _{\partial \Omega} f-\min _{\bar{\Omega}} f\right)} .
$$

Consequently, if $\mathrm{N}_{0} \geq 2$, the principal barrier of $f$ in $\Omega$ is thus degenerate and equal $\min _{\partial \Omega} f-$ $\min _{\bar{\Omega}} f$. Point $a$ in (H2) is convenient for computations, it can be relaxed using a similar procedure to [43] (see also [4]). When point $b$ in (H2) is satisfied, each $z \in \partial \mathrm{C}_{j} \cap \partial \Omega(j \in\{1, \ldots, \mathrm{N}\}$ ) such that $|\nabla f(z)|=0$ is a nondegenerate global minimum of $\left.f\right|_{\partial \Omega}$ (see [30, Proposition 11]). In particular, under (H2), for all $j \in\{1, \ldots, \mathbf{N}\}, \partial \mathrm{C}_{j} \cap \partial \Omega$ contains a finite number of points. Point $b$ in (H2) will be needed to use the results of [30 in Section 2.7 and we think that without this assumption, the prefactors appearing in Theorem 1 below might not be valid anymore, as explained in [30, Section 1.4].

\subsection{Main result}

When the function $f$ satisfies the assumptions (H1) and (H2), one defines for $j \in\{1, \ldots, \mathbf{N}\}$ :

$$
\Theta_{j, 1}=\frac{\sum_{\substack{z \in \partial \mathcal{C}_{j} \cap \partial \Omega,|\nabla f(z)| \neq 0}} \frac{\partial_{\mathrm{n}} f(z)}{\pi^{\frac{1}{2}}}\left(\left.\operatorname{det} \operatorname{Hess} f\right|_{\partial \Omega}(z)\right)^{-\frac{1}{2}}}{\sum_{y \in \arg \min _{\overline{C_{j}}} f}(\operatorname{det} \operatorname{Hess} f(y))^{-\frac{1}{2}}} \text { and } \Theta_{j, 2}=\frac{\sum_{\substack{z \in \partial \mathcal{C}_{j} \cap \partial \Omega,|\nabla f(z)|=0}} \frac{\left|\mu_{z}\right|}{\pi}|\operatorname{det} \operatorname{Hess} f(z)|^{-\frac{1}{2}}}{\sum_{y \in \arg \min _{\overline{C_{j}}} f}(\operatorname{det} \operatorname{Hess} f(y))^{-\frac{1}{2}}}
$$

where $\mu_{z}$ is the negative eigenvalue of Hess $f(z)$. The main result of this work is the following.

Theorem 1. Let $f: \bar{\Omega} \rightarrow \mathbb{R}$ be such that the assumptions (H1) and (H2) are satisfied. Then:

1. Let $\mathrm{K}$ be a compact subset of $\Omega$. Then, for any $\delta>0$, it holds for $h$ small enough:

$$
\sup _{x \in \mathrm{K}} \mathbb{E}_{x}\left[\tau_{\Omega^{c}}\right] \leq e^{\frac{2}{h}\left(\min _{\partial \Omega} f-\min _{\bar{\Omega}} f+\delta\right)} .
$$

2. Let $j \in\left\{1, \ldots, \mathrm{N}_{0}\right\}$ (see (8)) and $\mathrm{K}$ be a compact subset of $\mathcal{A}_{\Omega}\left(\mathrm{C}_{j}\right)$ (see (5)). Then, for all $x \in \mathrm{K}$, it holds in the limit $h \rightarrow 0$ :

$$
\mathbb{E}_{x}\left[\tau_{\Omega^{c}}\right]=\frac{e^{\frac{2}{h}\left(\min _{\partial \Omega} f-\min _{\bar{\Omega}} f\right)}}{\Theta_{j, 1} h^{-\frac{1}{2}}(1+O(h))+\Theta_{j, 2}(1+O(\sqrt{h}))} \text {, uniformly with respect to } x \in \mathrm{K} \text {. }
$$

3. Let $j \in\left\{\mathrm{N}_{0}+1, \ldots, \mathrm{N}\right\}$ (with the convention that $\left\{\mathrm{N}_{0}+1, \ldots, \mathrm{N}_{0}\right\}=\emptyset$ ) and $\mathrm{K}$ be a compact subset of $\mathcal{A}_{\Omega}\left(\mathrm{C}_{j}\right)$. Then, it holds for $h$ small enough:

$$
\min _{x \in \mathrm{K}} \mathbb{E}_{x}\left[\tau_{\Omega^{c}}\right] \geq \frac{e^{\frac{2}{h}\left(\min _{\partial \Omega} f-\min _{\overline{c_{j}}} f\right)}}{\Theta_{j, 1} h^{-\frac{1}{2}}(1+O(h))+\Theta_{j, 2}(1+O(\sqrt{h}))} .
$$

Furthermore, there exists $\varepsilon>0$, such that if $\min _{\overline{C_{j}}} f-\min _{\bar{\Omega}} f<\varepsilon$, then, when $h \rightarrow 0$ :

$$
\mathbb{E}_{x}\left[\tau_{\Omega^{c}}\right]=\frac{e^{\frac{2}{h}\left(\min _{\partial \Omega} f-\min _{\overline{c_{j}}} f\right)}}{\Theta_{j, 1} h^{-\frac{1}{2}}(1+O(h))+\Theta_{j, 2}(1+O(\sqrt{h}))} \text {, uniformly with respect to } x \in \mathrm{K} \text {. }
$$


As explained above, in many applications where the dynamics (1) is used, $\Omega$ is a basin of attraction of some local minimum of $f$ on $\mathbb{R}^{d}$, and therefore, for all $j,\left\{z \in \partial \mathrm{C}_{j} \cap \partial \Omega,|\nabla f(z)| \neq\right.$ $0\}=\emptyset$. Thus only the saddle points of $f$ appear (through the constants $\Theta_{j, 2}$ ) in the equivalents of $\mathbb{E}_{x}\left[\tau_{\Omega^{c}}\right]$ above. This is the main novelty of this work.

The remainder terms $O(h)$ and $O(\sqrt{h})$ in items 2 and 3 in Theorem 1 are optimal, as shown in Appendix 1 below. The second statement in item 3 in Theorem 1 can be seen as a perturbation result of the completely degenerate case $\mathrm{N}_{0}=\mathrm{N}$ (i.e. when all the $C_{\ell}$ 's have the same depth, namely $\left.\min _{\bar{\Omega}} f\right)$. Though it is assumed that $\bar{C}_{j}$ is a connected component of $\left\{f \leq \min _{\partial \Omega} f\right\}$ (and thus for all $i \neq j, \partial \mathrm{C}_{i} \cap \partial \mathrm{C}_{j}=\emptyset$ ), when $j \in\left\{\mathrm{N}_{0}+1, \ldots, \mathrm{N}\right\}$, a tunnelling effect can occur between $C_{j}$ and the others $C_{\ell}$ 's, mixing their properties. This is discussed in Appendix 2 below where we show that when, for some $j \geq \mathrm{N}_{0}+1, \min _{\overline{C_{j}}} f$ is much larger than $\min _{\bar{\Omega}} f$, $\lim _{h \rightarrow 0} h \ln \mathbb{E}_{x}\left[\tau_{\Omega^{c}}\right]>2\left(\min _{\partial \Omega} f-\min _{\overline{\mathrm{C}_{j}}} f\right)$, when $x \in \mathrm{C}_{j}$. Finally, as suggested by computations on one dimensional examples, when $\overline{C_{j}}$ is not a connected component of $\left\{f \leq \min _{\partial \Omega} f\right\}$, a strong tunnelling effect occurs between $C_{j}$ and the other $C_{i}$ 's which are such that $\partial C_{i} \cap \partial C_{j} \neq \emptyset$, and it is much harder to predict the precise asymptotic equivalents of $\mathbb{E}_{x}\left[\tau_{\Omega^{c}}\right]$ when $x \in \mathrm{C}_{j}$ due to the various situations that happen.

Remark 2. The prefactor $\Theta_{j, 1} h^{-\frac{1}{2}}$ in Theorem 1 differs from the one derived initially in [25]. Indeed, when $z \in \partial \mathrm{C}_{j} \cap \partial \Omega$ and $|\nabla f(z)| \neq 0$, it holds $\partial_{\mathrm{n}} f\left(z_{j}\right) \neq \mathrm{1}^{1}$ and thus, the Laplace's method on which is based the proof of Theorem 1 leads to a dependence on $h$ in this prefactor. The constant $\Theta_{j, 2}$ in Theorem 1 is the same as the one obtained in [25] up to a multiplication by 2 (see also [6. Theorem 3.2]): this is expected. Indeed, when the process (1) reaches a critical point $z \in \partial \mathrm{C}_{j} \cap \partial \Omega$, because $z$ is a saddle point of $f$, it has, in the limit $h \rightarrow 0$, a one-half probability to come back in $\Omega$ and a one-half probability to go in $\mathbb{R}^{d} \backslash \bar{\Omega}$ (this result is not difficult to prove in dimension 1, see [42, Section A.1.2.2], and can be extended to higher dimensions using a suitable set of coordinates around $z$ ).

According to items 2 and 3 in Theorem 1 , for $x, y \in \mathcal{A}_{\Omega}\left(\mathrm{C}_{j}\right), \mathbb{E}_{x}\left[\tau_{\Omega^{c}}\right]=\mathbb{E}_{y}\left[\tau_{\Omega^{c}}\right](1+o(1))$ when $h \rightarrow 0$, where $o(1)$ either equal $O(\sqrt{h})$ or $O(h)$. We will actually derive the following more precise leveling result on $x \in \Omega \mapsto \mathbb{E}_{x}\left[\tau_{\Omega^{c}}\right]$.

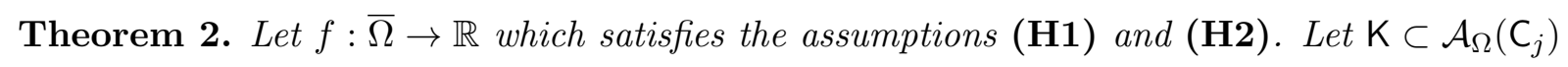
for some $j \in\{1, \ldots, \mathrm{N}\}$. Then, if $j \in\left\{1, \ldots, \mathrm{N}_{0}\right\}$ (see (8)), there exists $c>0$ such that for $h$ small enough and for all $x, y \in \mathrm{K}$ :

$$
\mathbb{E}_{x}\left[\tau_{\Omega^{c}}\right]=\mathbb{E}_{y}\left[\tau_{\Omega^{c}}\right]\left(1+O\left(e^{-\frac{c}{h}}\right)\right) \text {, uniformly in } x, y \in \mathrm{K} .
$$

Moreover, if $j \geq \mathrm{N}_{0}+1$, there exists $\varepsilon>0$, such that if $\min _{\overline{\mathrm{C}_{j}}} f-\min _{\bar{\Omega}} f<\varepsilon$, there exists $c>0$, such that for $h$ small enough for all $x, y \in \mathrm{K}$ :

$$
\mathbb{E}_{x}\left[\tau_{\Omega^{c}}\right]=\mathbb{E}_{y}\left[\tau_{\Omega^{c}}\right]\left(1+O\left(e^{-\frac{c}{h}}\right)\right) \text {, uniformly in } x, y \in \mathrm{K} .
$$

Theorem 2 generalizes, as long as the gradient case is concerned, the leveling result 9 , Corollary 1] to the case when $f$ has critical points on $\partial \Omega$ and several critical points in $\Omega$.

To prove Theorem 1, the starting point is a formula from the potential theory for $\mathbb{E}_{x_{*}}\left[\tau_{\Omega^{c}}\right]$ when $x_{*}$ is a local minimum of $f$ in $\Omega$. To extract information from this formula, we derive

\footnotetext{
${ }^{1}$ Such points $z$ have also been considered by Kramers in 25 to derive formulas for transition rates, as explained in 40 .
} 
leveling properties on the mean exit time from $\Omega$ and commitor functions, and we give sharp equivalents on capacities: this is the purpose of Section 2 where more general assumptions than (H1) and (H2) are considered. Then, in Section 3, we prove Theorems 1 and 2, Let us mention that our analysis is not a straightforward extension of the analysis led in [6, because in our setting, we have to analyse the effect of the boundary $\partial \Omega$ on the sharp equivalents of $\mathbb{E}_{x}\left[\tau_{\Omega^{c}}\right]$ when $x \in \Omega$ and $h \rightarrow 0$. Moroever, we also consider a set of deterministic initial conditions in $\Omega$ with nonzero Lebesgue measure (here $\left.\mathcal{A}_{\Omega}\left(\mathrm{C}_{j}\right), j \in\{1, \ldots, \mathrm{N}\}\right)$ and not only the case when $X_{0}=x_{*}$ (which requires a further analysis, see Sections 3.2 and 3.3 ). This is important for the following reason. In many applications where the dynamics (1) is used, one is interested in having a sharp estimate on the average time this process remains trapped in $\Omega$ when $h \ll 1$. Since the process (1) has a density with respect to the Lebesgue measure in $\mathbb{R}^{d}$, for all $h>0$, the probability that the trajectories of (1) entering in $\Omega$ pass through each $x_{*}$ is zero.

Let us mention that our analysis to prove Theorem 1 will allow us to derive Corollary 10 which states in particular that, when $\partial \mathrm{C}(x) \cap \partial \Omega=\{z\}$ (see (6)) and $\overline{\mathrm{C}(x)}$ is a connected component of $\{f \leq \lambda(x)\}$, the process (1) exits $\Omega$ almost surely through any neighborhood of $z$ in the limit $h \rightarrow 0$ when $X_{0}=x \in \mathcal{A}_{\Omega}(\mathrm{C}(x))$. Since $z$ can be a critical point of $f$, to the best of our knowledge, Corollary 10 is new for the dynamics (1) (see indeed [10] or [32, Section 1]).

\subsection{Link with the previous results on the mean exit time}

Let us now recall the main previous contributions on the study of $\mathbb{E}_{x}\left[\tau_{\Omega^{c}}\right]$. The limit of $h \ln \mathbb{E}\left[\tau_{\Omega^{c}}\right]$ when $h \rightarrow 0$ and $x \in \Omega$ has been studied by Freidlin and Wentzell in [17] when $|\nabla f| \neq 0$ on $\partial \Omega$ (see also [36]). In [9], it is shown that, for $x \in \Omega, \mathbb{E}_{x}\left[\tau_{\Omega^{c}}\right] \lambda_{1, h}=1+o(1)$ when $h \rightarrow 0$, in the case when $\partial_{\mathrm{n}} f>0$ on $\partial \Omega$ and $f$ has a unique critical point in $\Omega$ (see also [43]). We also refer to 23,24] for the study, when $|\nabla f| \neq 0$ on $\partial \Omega$, of the solution of the parabolic equation $\mathcal{L}_{f, h} \mathrm{u}_{h}=\partial_{t} \mathrm{u}_{h}$ as $h \rightarrow 0$. Let us mention that in $[9,17,23,24,36]$, the authors also considered non reversible processes. Sharp asymptotic estimates when $h \rightarrow 0$ of $\mathbb{E}_{x}\left[\tau_{\Omega^{c}}\right]$ for some $x \in \Omega$ have been obtained in $[50$, Section 4] when $|\nabla f| \neq 0$ on $\partial \Omega$ (let us also mention that the assumption that $\partial\left\{f<\min _{\partial \Omega} f\right\} \cap \partial \Omega \neq \emptyset$ is not allowed there according to the assumption (C3) in 50, Section 4]). In [37, Theorem 5], when $f$ has a non degenerate principal barrier (i.e. in our setting, when $\mathrm{N}_{0}=1$ ), it is shown that $\lambda_{1, h} \tau_{\Omega^{c}}$ converges in distribution to an exponential random variable in the limit $h \rightarrow 0$ when $X_{0}=x$ for some $x \in \Omega$ (in our setting, for $x \in \mathrm{C}_{1}$ ). A comprehensive review of the literature on this topic can be found in [1]. We refer to [35, 38, 40, 46] where asymptotic formulas for $\mathbb{E}\left[\tau_{\Omega^{c}}\right]$ when $h \rightarrow 0$ have been obtained through formal computations in different settings (see also 12,45 ). We finally mention $[2,3,6,7,18,22,26,28,41$, and references therein for asymptotics estimates on eigenvalues, transition times in the boundary less case.

\section{General results on commitor functions and capacities}

In all this section, we assume that the function $f: \bar{\Omega} \rightarrow \mathbb{R}$ is a $\mathcal{C}^{\infty}$ Morse function. In this section, we derive leveling results on commitor functions and we give asymptotic equivalents of capacities in the limit $h \rightarrow 0$. 


\subsection{The commitor function}

For any closed ball $\mathrm{B} \subset \Omega$, one denotes by

$$
\tau_{\mathrm{B}}=\inf \left\{t \geq 0 \mid X_{t} \in \mathrm{B}\right\}
$$

the first time the process (1) hits B. Let $x_{*}$ a local minimum of $f$ in $\Omega$. In all this work, we consider for all $h>0$ a constant $r_{h}>0$ such that when $h \rightarrow 0$,

$$
r_{h}=O\left(e^{-\frac{\delta_{*}}{h}}\right),
$$

where $\delta_{*}>0$ will be fixed in Lemma 3. In the following, $h>0$ is chosen small enough so that $\mathrm{B}_{r_{h}}\left(x_{*}\right) \subset \mathrm{C}\left(x_{*}\right)$. The commitor function (also called equilibrium potential) between $\mathrm{B}_{r_{h}}\left(x_{*}\right)$ and $\Omega^{c}$ is defined by:

$$
\mathrm{p}_{x_{*}}: x \in \bar{\Omega} \mapsto \mathbb{P}_{x}\left[\tau_{\mathrm{B}_{r_{h}}\left(x_{*}\right)}<\tau_{\Omega^{c}}\right] .
$$

A schematic representation of $\mathrm{C}\left(x_{*}\right)$ and $\mathrm{B}_{r_{h}}\left(x_{*}\right)$ is given in Figure 2 when $\partial \mathrm{C}\left(x_{*}\right) \cap \partial \Omega \neq \emptyset$.

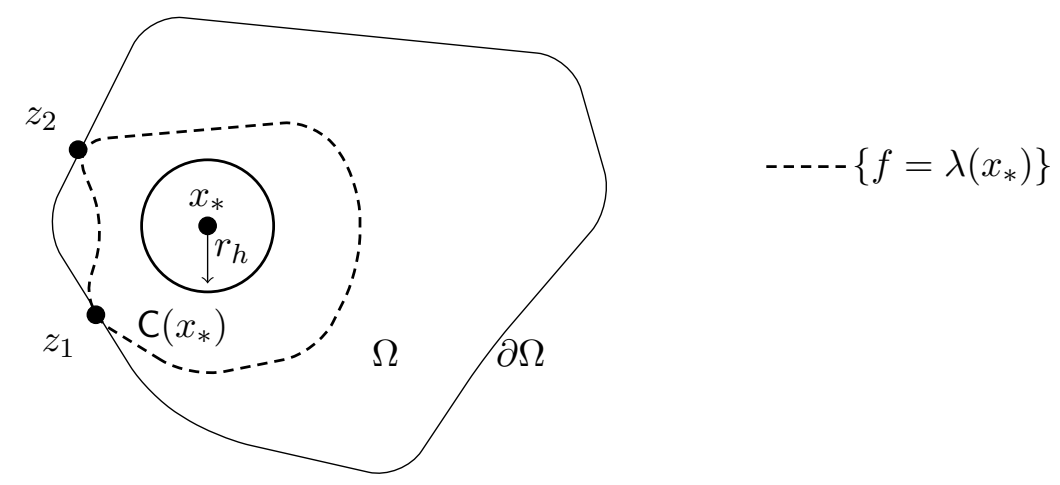

Figure 2: Schematic representation of $\mathrm{C}\left(x_{*}\right)$ (see (6)) and $\mathrm{B}_{r_{h}}\left(x_{*}\right)$. On the figure $\partial \mathrm{C}\left(x_{*}\right) \cap \partial \Omega$ is non empty and equals $\left\{z_{1}, z_{2}\right\}$.

\subsection{A formula from the potential theory}

In this section, we recall a formula from the potential theory which is used in this work (see (11)), which can be found for instance in [5]. Let us denote by $G_{\Omega}$ be the Green function of $\mathcal{L}_{f, h}$ associated with homogeneous Dirichlet boundary conditions on $\partial \Omega$. The equilibrium measure $e_{\mathrm{B}_{r_{h}}\left(x_{*}\right), \Omega^{c}}$ associated with the couple $\left(\mathrm{B}_{r_{h}}\left(x_{*}\right), \Omega^{c}\right)$ (see [6, Section 2] and more precisely the equation (2.10) there) is defined as the unique measure on $\partial \mathrm{B}_{r_{h}}\left(x_{*}\right)$ such that

$$
\mathrm{p}_{x_{*}}(x)=\int_{\partial \mathrm{B}_{r_{h}}\left(x_{*}\right)} G_{\Omega}(x, y) e_{\mathrm{B}_{r_{h}}\left(x_{*}\right), \Omega^{c}}(d y) .
$$

From [6, Section 2] (see equation (2.27) there), one has the following relation:

$$
\int_{\partial \mathrm{B}_{r_{h}}\left(x_{*}\right)} \mathbb{E}_{z}\left[\tau_{\Omega^{c}}\right] e^{-\frac{2}{h} f(z)} e_{\mathrm{B}_{r_{h}}\left(x_{*}\right), \Omega^{c}}(d z)=\int_{\Omega} e^{-\frac{2}{h} f(x)} \mathrm{p}_{x_{*}}(x) d x,
$$

where $p_{x_{*}}$ is defined by (10). Let us now define, as in [6, Section 2] (see equation (2.13) there), the capacity associated with $\left(\mathrm{B}_{r_{h}}\left(x_{*}\right), \Omega^{c}\right)$ :

$$
\operatorname{cap}_{\mathrm{B}_{r_{h}}\left(x_{*}\right)}\left(\Omega^{c}\right)=\int_{\partial \mathrm{B}_{r_{h}}\left(x_{*}\right)} e^{-\frac{2}{h} f(z)} e_{\mathrm{B}_{r_{h}}\left(x_{*}\right), \Omega^{c}}(d z) .
$$


Finally, let us recall that from [6, Section 2], one has the following variational principle:

$$
\operatorname{cap}_{\mathrm{B}_{r_{h}}\left(x_{*}\right)}\left(\Omega^{c}\right)=\frac{h}{2} \int_{\Omega \backslash \mathrm{B}_{r_{h}}\left(x_{*}\right)}\left|\nabla \mathrm{p}_{x_{*}}\right|^{2} e^{-\frac{2}{h} f}=\inf _{\mathbf{p} \in \mathcal{H}_{\mathrm{B}_{r_{h}}\left(x_{*}\right), \Omega^{c}}} \frac{h}{2} \int_{\Omega \backslash \mathrm{B}_{r_{h}}\left(x_{*}\right)}|\nabla \mathrm{p}|^{2} e^{-\frac{2}{h} f},
$$

where we recall $\mathrm{p}_{x_{*}}$ is defined by $(10)$ and

$$
\mathcal{H}_{\mathrm{B}_{r_{h}}\left(x_{*}\right), \Omega^{c}}=\left\{\mathrm{p} \in H^{1}\left(\Omega \backslash \mathrm{B}_{r_{h}}\left(x_{*}\right), e^{-\frac{2}{h} f} d x\right), \mathrm{p}=1 \text { on } \partial \mathrm{B}_{r_{h}}\left(x_{*}\right), \mathrm{p}=0 \text { on } \partial \Omega\right\} .
$$

The second equality in $(13)$ holds since the function $\mathrm{p}_{x_{*}}$ is a minimizer of the functional

$$
\mathrm{p} \in \mathcal{H}_{\mathrm{B}_{r_{h}}\left(x_{*}\right), \Omega^{c}} \mapsto \frac{h}{2} \int_{\Omega \backslash \mathrm{B}_{r_{h}}\left(x_{*}\right)}|\nabla \mathrm{p}|^{2} e^{-\frac{2}{h} f} .
$$

\subsection{Leveling results on the mean exit time}

In order to extract $\mathbb{E}_{x_{*}}\left[\tau_{\Omega^{c}}\right]$ from the left hand side of $[11)$, we need a leveling result on the function $z \mapsto \mathbb{E}_{z}\left[\tau_{\Omega^{c}}\right]$ in $\mathrm{B}_{r_{h}}\left(x_{*}\right)$. This the purpose of the next lemma.

Lemma 3. Let $f: \bar{\Omega} \rightarrow \mathbb{R}$ be a $\mathcal{C}^{\infty}$ Morse function. Let $x_{*}$ be a local minimum of $f$ in $\Omega$ and $r_{h}>0$ such that (9) holds for some $\delta_{*}>0$. Then, there exist $h_{0}>0$ and $c>0$ such that for all $h \in\left(0, h_{0}\right)$ it holds

$$
\max _{z \in \mathrm{B}_{r_{h}}\left(x_{*}\right)}\left|\mathbb{E}_{z}\left[\tau_{\Omega^{c}}\right]-\mathbb{E}_{x_{*}}\left[\tau_{\Omega^{c}}\right]\right| \leq e^{-\frac{c}{h}} \mathbb{E}_{x_{*}}\left[\tau_{\Omega^{c}}\right] .
$$

Proof. Let us recall that from the Dynkin's formula, the function $w: x \mapsto \mathbb{E}_{z}\left[\tau_{\Omega^{c}}\right]$ is the unique weak solution to

$$
\mathcal{L}_{f, h} w=1 \text { in } \Omega \text { and } w=0 \text { on } \partial \Omega,
$$

and $w \in \mathcal{C}^{\infty}(\bar{\Omega}, \mathbb{R})$. Set $b:=\nabla f$. For $z \in \mathrm{B}_{2 \sqrt{h}}\left(x_{*}\right)$, set $y=\left(z-x_{*}\right) / \sqrt{h}+x_{*} \in \mathrm{B}_{2}\left(x_{*}\right)$ and $v(y)=w\left(\left(y-x_{*}\right) \sqrt{h}+x_{*}\right)$ which satisfies for $y \in \mathrm{B}_{2}\left(x_{*}\right)$,

$$
-\frac{1}{2} \Delta v(y)+\frac{b\left(\left(y-x_{*}\right) \sqrt{h}+x_{*}\right)}{\sqrt{h}} \cdot \nabla v(y)=1 .
$$

Then, since $b\left(x_{*}\right)=0$ (and thus $y \mapsto b\left(\left(y-x_{*}\right) \sqrt{h}+x_{*}\right) / \sqrt{h}$ on $\mathrm{B}_{2}\left(x_{*}\right)$ is bounded uniformly with respect to $h)$, using the Schauder estimate 19 , Corollary 6.3] on $y \mapsto v(y)$ on $\mathrm{B}_{2}\left(x_{*}\right)$, there exits $C>0$ independent of $h$ such that,

$$
\max _{y \in \mathrm{B}_{1}\left(x_{*}\right)}|\nabla v(y)| \leq C\left(1+\max _{y \in \mathrm{B}_{2}\left(x_{*}\right)} v(y)\right) .
$$

Thus, coming back to the variable $z$, it holds:

$$
\max _{z \in \mathrm{B}_{\sqrt{h}}\left(x_{*}\right)}\left|\nabla \mathbb{E}_{z}\left[\tau \Omega^{c}\right]\right| \leq \frac{C}{\sqrt{h}}\left(1+\max _{z \in \mathrm{B}_{2 \sqrt{h}}\left(x_{*}\right)} \mathbb{E}_{z}\left[\tau \Omega^{c}\right]\right) .
$$

Moreover, there exist $M>0$ and $c>0$ such that for $h$ small enough and for all $z \in \mathrm{B}_{2 \sqrt{h}}\left(x_{*}\right)$, $\mathbb{E}_{z}\left[\tau_{\Omega^{c}}\right] \geq M e^{\frac{c}{h}}$. Indeed, this follows from [16, Lemma 1] together with the fact that for all $z \in \mathrm{B}_{2 \sqrt{h}}\left(x_{*}\right), \mathbb{E}_{z}\left[\tau_{\Omega^{c}}\right] \geq \mathbb{E}_{z}\left[\tau_{\mathrm{U}^{c}}\right]$ where $\mathrm{U}$ is a $\overline{\mathcal{C}^{\infty}}$ subdomain of $\Omega$ such that $\partial_{\mathrm{n}} f>0$ on $\partial \mathrm{U}$ and $\{z \in \mathrm{U}, \nabla f(z)=0\}=\left\{x_{*}\right\}$ (such a $\mathrm{U}$ exists since $x_{*}$ is a nondegenerate local minimum of $f$ in $\Omega$ ). Hence, one has, for $h$ small enough,

$$
\max _{z \in \mathrm{B}_{\sqrt{h}}\left(x_{*}\right)}\left|\nabla \mathbb{E}_{z}\left[\tau_{\Omega^{c}}\right]\right| \leq \frac{C}{\sqrt{h}} \max _{z \in \mathrm{B}_{2 \sqrt{h}}\left(x_{*}\right)} \mathbb{E}_{z}\left[\tau_{\Omega^{c}}\right]
$$


Then, for all $z \in \mathrm{B}_{\sqrt{h}}\left(x_{*}\right)$,

$$
\left|\mathbb{E}_{z}\left[\tau_{\Omega^{c}}\right]-\mathbb{E}_{x_{*}}\left[\tau_{\Omega^{c}}\right]\right| \leq \frac{C}{\sqrt{h}}\left|z-x_{*}\right| \max _{z \in \mathrm{B}_{2 \sqrt{h}}\left(x_{*}\right)} \mathbb{E}_{z}\left[\tau_{\Omega^{c}}\right],
$$

and thus, for all $z \in \mathrm{B}_{r_{h}}\left(x_{*}\right)$,

$$
\left|\mathbb{E}_{z}\left[\tau_{\Omega^{c}}\right]-\mathbb{E}_{x_{*}}\left[\tau_{\Omega^{c}}\right]\right| \leq C \frac{e^{-\frac{\delta_{*}}{h}}}{\sqrt{h}} \max _{z \in \mathrm{B}_{2 \sqrt{h}}\left(x_{*}\right)} \mathbb{E}_{z}\left[\tau_{\Omega^{c}}\right] .
$$

Finally, it follows from [6, Equation (6.3)] (see also [26, Lemma 9.1]) that it holds:

$$
\max _{z \in \mathrm{B}_{2 \sqrt{h}}\left(x_{*}\right)} \mathbb{E}_{z}\left[\tau_{\Omega^{c}}\right] \leq C \min _{z \in \mathrm{B}_{2 \sqrt{h}}\left(x_{*}\right)} \mathbb{E}_{z}\left[\tau_{\Omega^{c}}\right]
$$

for some $C>0$. In conclusion, one has for $h$ small enough, $\max _{z \in \mathrm{B}_{r_{h}}\left(x_{*}\right)}\left|\mathbb{E}_{z}\left[\tau_{\Omega^{c}}\right]-\mathbb{E}_{x_{*}}\left[\tau_{\Omega^{c}}\right]\right| \leq$ $C e^{-\frac{\delta_{*}}{2 h}} \mathbb{E}_{x_{*}}\left[\tau_{\Omega^{c}}\right]$. This concludes proof of Lemma 3 .

Using Lemma 3 together with (11) and (12), one obtains, for all local minima $x_{*}$ of $f$ in $\Omega$, in the limit $h \rightarrow 0$ :

$$
\mathbb{E}_{x_{*}}\left[\tau_{\Omega^{c}}\right]=\frac{\int_{\Omega} e^{-\frac{2}{h} f(x)} \mathrm{p}_{x_{*}}(x) d x}{\operatorname{cap}_{\mathrm{B}_{r_{h}}\left(x_{*}\right)}\left(\Omega^{c}\right)}\left(1+O\left(e^{-\frac{c}{h}}\right)\right), \text { for some } c>0 \text { independent of } h .
$$

To compute a sharp asymptotic equivalent of $\mathbb{E}_{x_{*}}\left[\tau_{\Omega^{c}}\right]$ when $h \rightarrow 0$, we study the asymptotic behaviour of $\mathrm{p}_{x_{*}}(x)$ and $\operatorname{cap}_{\mathrm{B}_{r_{h}}\left(x_{*}\right)}\left(\Omega^{c}\right)$ in the limit $h \rightarrow 0$. This is the purpose of the next sections.

\subsection{Leveling results on the commitor function $\mathrm{p}_{x_{*}}$ in $\mathrm{C}\left(x_{*}\right)$}

Let us prove the following leveling property on the commitor function $\mathrm{p}_{x_{*}}$ (see $(10 p)$ in a $h$ independent neighborhood $\mathrm{U}$ of $x_{*}$ in $\mathrm{C}\left(x_{*}\right)$ (see (6) ).

Lemma 4. Let $f: \bar{\Omega} \rightarrow \mathbb{R}$ be a $\mathcal{C}^{\infty}$ Morse function. Let $x_{*}$ be a local minimum of $f$ in $\Omega$. Let $\mathrm{U}$ be $a \mathcal{C}^{\infty}$ subdomain of $\mathrm{C}\left(x_{*}\right)$ (see (6)) such that $\partial_{\mathrm{n}} f>0$ on $\partial \mathrm{U}$ and $\{z \in \mathrm{U}, \nabla f(z)=0\}=\left\{x_{*}\right\}$. Let $\mathrm{K}$ be a $\mathcal{C}^{\infty}$ compact subset of $\mathrm{U}$. Then, there exists $\delta_{*}>0$ (see (9)) such that there exist $h_{0}>0$ and $c>0$, for all $h \in\left(0, h_{0}\right)$ :

$$
\left.\max _{y \in \mathrm{K}}\left|\mathbf{p}_{x_{*}}(y)-1\right| \leq e^{-\frac{c}{h}} \text {, where we recall } \mathbf{p}_{x_{*}} \text { is defined in } 10\right) \text {. }
$$

Proof. Since the trajectories of the process (1) are continuous, one has for all $y \in \mathrm{K},\left\{\tau_{\Omega^{c}}<\right.$ $\left.\tau_{\mathrm{B}_{r_{h}}\left(x_{*}\right)}\right\} \subset\left\{\tau_{\mathrm{U}^{c}}<\tau_{\mathrm{B}_{r_{h}}\left(x_{*}\right)}\right\}$ when $X_{0}=y$. Using [8. Theorem 2], there exist $\delta_{*}>0$ (see (9)), $h_{0}>0$ and $c>0$ such that for all $h \in\left(0, h_{0}\right)$ and $\left.y \in \mathrm{K}, \mathbb{P}_{y}\left[\tau_{\cup^{c}} \geq \tau_{\mathrm{B}_{r_{h}}\left(x_{*}\right)}\right\}\right] \leq e^{-\frac{c}{h}}$. This concludes the proof of Lemma 4.

With Lemma 4, we can extend the leveling property on $\mathrm{p}_{x_{*}}$ to $\mathcal{A}_{\Omega}\left(\mathrm{C}\left(x_{*}\right)\right)$. 
Proposition 5. Let $f: \bar{\Omega} \rightarrow \mathbb{R}$ be a $\mathcal{C}^{\infty}$ Morse function. Let $x_{*}$ be a local minimum of $f$ in $\Omega$. Let $\mathrm{K}$ be a compact subset of $\mathcal{A}_{\Omega}\left(\mathrm{C}\left(x_{*}\right)\right.$ ) (see (6) and (5)). Then, there exist $h_{0}>0$ and $c>0$ such that for all $h \in\left(0, h_{0}\right)$, one has:

$$
\max _{y \in \mathrm{K}}\left|\mathrm{p}_{x_{*}}(y)-1\right| \leq e^{-\frac{c}{h}}
$$

This implies in particular that, using a Laplace's method, for all $k \in\{1,2\}$, in the limit $h \rightarrow 0$ :

$$
\int_{\mathrm{C}\left(x_{*}\right)} \mathbf{p}_{x_{*}}^{k} e^{-\frac{2}{h} f}=(h \pi)^{\frac{d}{2}} e^{-\frac{2}{h} \min _{\bar{C}\left(x_{*}\right)} f} \sum_{y \in \arg \min \frac{\bar{C}_{\left(x_{*}\right)}}{}}(\operatorname{det} \operatorname{Hess} f(y))^{-\frac{1}{2}}(1+O(h)) .
$$

Proof. Let us introduce the function $\mathrm{q}_{x_{*}}: y \mapsto 1-\mathrm{p}_{x_{*}}(y)$ which is the $\mathcal{C}^{\infty}\left(\overline{\Omega \backslash \mathrm{B}_{r_{h}}\left(x_{*}\right)}, \mathbb{R}\right)$ solution to

$$
-\frac{h}{2} \Delta \mathbf{q}_{x_{*}}=-\nabla f \cdot \nabla \mathbf{q}_{x_{*}} \text { in } \Omega \backslash \mathrm{B}_{r_{h}}\left(x_{*}\right), \mathbf{q}_{x_{*}}=1 \text { on } \partial \Omega \text { and } \mathbf{q}_{x_{*}}=0 \text { on } \partial \mathrm{B}_{r_{h}}\left(x_{*}\right) .
$$

Working with $\mathrm{q}_{x_{*}}$ instead of $\mathrm{p}_{x_{*}}$ will be convenient to derive the key estimate (25) below. The proof of Proposition 5 is divided into several steps. The two first steps are inspired by [12] but we have to take care here with the regularity of $\mathrm{q}_{x_{*}}$ which does not belong to $H^{2}\left(\mathrm{C}\left(x_{*}\right)\right)$. For that reason, before starting the proof of Proposition 5, we introduce the following sets. Let $r_{*}>0$ and $\mathrm{U}$ be a smooth subdomain of $\mathrm{C}\left(x_{*}\right)$ as in Lemma 4 with moreover $\overline{\mathrm{U}} \subset \mathrm{C}\left(x_{*}\right)$, such that:

$$
\mathrm{B}_{r_{*}}\left(x_{*}\right) \subset \mathrm{U} .
$$

For $m \in \mathbb{N}^{*}$, we consider a sequence

$$
\left(r_{k}\right)_{k=1, \ldots, m} \in\left(\mathbb{R}_{+}^{*}\right)^{m} \text { such that } 0<r_{1}<r_{2}<\ldots<r_{m}<r_{m+1}:=r_{*} .
$$

It then follows that $\mathrm{B}_{r_{k}}\left(x_{*}\right) \subset \operatorname{int}\left(\mathrm{B}_{r_{k+1}}\left(x_{*}\right)\right)$, for $k=1, \ldots, m$. In the following, $h>0$ is chosen small enough so that

$$
\mathrm{B}_{r_{h}}\left(x_{*}\right) \subset \operatorname{int}\left(\mathrm{B}_{r_{1}}\left(x_{*}\right)\right) .
$$

In the following, $C>0$ is a constant which can change from one occurrence to another and which does not depend on $h$. We are now in position to prove Proposition 5 .

Step 1: Let us prove that there exist $h_{0}>0, \gamma>0$ and $C>0$ such that for all $h \in\left(0, h_{0}\right)$,

$$
\left\|\nabla \mathbf{q}_{x_{*}}\right\|_{L^{\infty}\left(\Omega \backslash \mathrm{B}_{r_{*}}\left(x_{*}\right)\right)} \leq C h^{-\gamma} .
$$

To this end, we apply recursively $H^{2}$-interior elliptic estimates outside a neighboorhood of $\mathrm{B}_{r_{h}}\left(x_{*}\right)$. This will allow us to use Sobolev injections with constants independent of $h$. Using (16) and combining $H^{2}$-interior elliptic estimates and $H^{2}$-boundary elliptic estimates nea $\partial \Omega$ (see 15 , Theorem 1 and Theorem 4 in Section 6.3]), there exists $C>0$ such that:

$$
\left\|\mathrm{q}_{x_{*}}\right\|_{H^{2}\left(\Omega \backslash \mathrm{B}_{r_{2}}\left(x_{*}\right)\right)} \leq C\left(1+h^{-1}\left\|\nabla \mathrm{q}_{x_{*}}\right\|_{L^{2}\left(\Omega \backslash \mathrm{B}_{r_{1}}\left(x_{*}\right)\right)}\right) .
$$

Moreover, using (16), the trace theorem and (21) together with the fact that $\mathbf{q}_{x_{*}}=0$ on $\partial \mathrm{B}_{r_{h}}\left(x_{*}\right)$ and $\mathrm{q}_{x_{*}}=1$ on $\partial \Omega$, there exists $C>0$ such that for any $\varepsilon>0$ and $\varepsilon^{\prime}>0$,

$$
\begin{aligned}
h\left\|\nabla \mathbf{q}_{x_{*}}\right\|_{L^{2}\left(\Omega \backslash \mathrm{B}_{r_{h}}\left(x_{*}\right)\right)}^{2} & \leq C\left(h \int_{\partial \Omega}\left|\partial_{\mathrm{n}} \mathbf{q}_{x_{*}}\right| d \sigma+\int_{\Omega \backslash \mathrm{B}_{r_{h}}\left(x_{*}\right)}\left|\nabla f \cdot \nabla \mathbf{q}_{x_{*}}\right|\right) \\
& \leq C\left(\frac{h}{\varepsilon}+h \varepsilon\left\|\mathbf{q}_{x_{*}}\right\|_{H^{2}\left(\Omega \backslash \mathrm{B}_{r_{2}}\left(x_{*}\right)\right)}^{2}+\frac{1}{\varepsilon^{\prime}}+\varepsilon^{\prime}\left\|\nabla \mathbf{q}_{x_{*}}\right\|_{L^{2}\left(\Omega \backslash \mathrm{B}_{r_{h}}\left(x_{*}\right)\right)}^{2}\right) \\
& \leq C\left(\frac{h}{\varepsilon}+h^{-1} \varepsilon\left(\left\|\nabla \mathbf{q}_{x_{*}}\right\|_{L^{2}\left(\Omega \backslash \mathrm{B}_{r_{1}}\left(x_{*}\right)\right)}^{2}+1\right)+\frac{1}{\varepsilon^{\prime}}+\varepsilon^{\prime}\left\|\nabla \mathbf{q}_{x_{*}}\right\|_{L^{2}\left(\Omega \backslash \mathrm{B}_{r_{h}}\left(x_{*}\right)\right)}^{2}\right) .
\end{aligned}
$$


Choosing $\varepsilon^{\prime}=\frac{h}{4(C+1)}$, one obtains

$$
\frac{3}{4} h\left\|\nabla \mathbf{q}_{x_{*}}\right\|_{L^{2}\left(\Omega \backslash \mathrm{B}_{r_{h}}\left(x_{*}\right)\right)}^{2} \leq C\left(\frac{h}{\varepsilon}+h^{-1} \varepsilon\left(\left\|\nabla \mathbf{q}_{x_{*}}\right\|_{L^{2}\left(\Omega \backslash \mathrm{B}_{r_{1}}\left(x_{*}\right)\right)}^{2}+1\right)+h^{-1}\right) .
$$

Thus, since $\Omega \backslash \mathrm{B}_{r_{1}}\left(x_{*}\right) \subset \Omega \backslash \mathrm{B}_{r_{h}}\left(x_{*}\right)$ (see 190 ),

$$
\frac{3 h}{4}\left\|\nabla \mathbf{q}_{x_{*}}\right\|_{L^{2}\left(\Omega \backslash \mathrm{B}_{r_{1}}\left(x_{*}\right)\right)}^{2} \leq C\left(\frac{h}{\varepsilon}+h^{-1} \varepsilon\left(\left\|\nabla \mathrm{q}_{x_{*}}\right\|_{L^{2}\left(\Omega \backslash \mathrm{B}_{r_{1}}\left(x_{*}\right)\right)}^{2}+1\right)+h^{-1}\right) .
$$

Then, one chooses $\varepsilon=\frac{h^{2}}{4(C+1)}$ which implies that $\left\|\nabla \mathrm{q}_{x_{*}}\right\|_{L^{2}\left(\Omega \backslash \mathrm{B}_{r_{1}}\left(x_{*}\right)\right)} \leq C h^{-1}$. Thus, from (21), there exist $C>0$ and $h_{0}>0$ such that for all $h \in\left(0, h_{0}\right)$ :

$$
\left\|\mathbf{q}_{x_{*}}\right\|_{H^{2}\left(\Omega \backslash \mathrm{B}_{r_{2}}\left(x_{*}\right)\right)} \leq C h^{-2} .
$$

Then, for $k \in\{3, \ldots, m\}$, using $H^{k}$-interior elliptic estimates and $H^{k}$-boundary elliptic estimates 15 , Theorems 4 and 5 in Section 6.3.2], one obtains recursively that there exists $C>0$ such that for $h$ small enough:

$$
\left\|\mathbf{q}_{x_{*}}\right\|_{H^{k}\left(\Omega \backslash \mathrm{B}_{r_{k}}\left(x_{*}\right)\right)} \leq C h^{-1}\left(1+\left\|\nabla \mathbf{q}_{x_{*}}\right\|_{H^{k-1}\left(\Omega \backslash \mathrm{B}_{r_{k}}\left(x_{*}\right)\right)}\right) \leq C h^{-k} .
$$

Then, choosing $m$ (see 18) such that $m>\frac{d}{2}$ yields 20 using the Sobolev injection $H^{m}\left(\Omega \backslash \mathrm{B}_{r_{m}}\left(x_{*}\right)\right)$ is continuously embedded in $L^{\infty}\left(\Omega \backslash \mathrm{B}_{r_{m}}\left(x_{*}\right)\right)$.

Step 2: Let us define for $\eta>0$ the set (see (6p)

$$
\mathrm{C}_{\eta}\left(x_{*}\right)=\mathrm{C}\left(x_{*}\right) \cap\left\{f<\lambda\left(x_{*}\right)-\eta\right\} \subset \Omega,
$$

Let

$$
c_{*, M}:=\max \left\{f(y), y \in \mathrm{C}\left(x_{*}\right) \text { such that }|\nabla f(y)|=0\right\} .
$$

Since $f$ is a Morse function on $\bar{\Omega}, f$ has a finite number of critical points in $\bar{\Omega}$, and thus: $c_{*, M}<\lambda\left(x_{*}\right)$. Let us consider

$$
\left.\left.\eta_{*} \in\left(0, \lambda\left(x_{*}\right)-c_{*, M}\right) \text { such that } \overline{\mathrm{U}} \subset \mathrm{C}_{\eta_{*}}\left(x_{*}\right) \text { (see } 117\right)-19\right) \text { ) and } \eta \in\left(0, \eta_{*}\right] \text {. }
$$

According to 30, Proposition 10], $\mathrm{C}_{\eta}\left(x_{*}\right)$ is a connected component of $\left\{f<\lambda\left(x_{*}\right)-\eta\right\}$. Moreover, $\mathrm{C}_{\eta}\left(x_{*}\right)$ is $\mathcal{C}^{\infty}$ because its boundary does not contain critical points of $f$ by choice of $\eta$ and $\mathrm{C}_{\eta}\left(x_{*}\right) \subset \Omega$. Let us now prove that for all $\eta_{0} \in\left(0, \eta_{*}\right]$, there exists $\alpha_{0}>0$ such that for $h$ small enough:

$$
\left\|\nabla \mathbf{q}_{x_{*}}\right\|_{L^{\infty}\left(\mathrm{C}_{\eta_{0}}\left(x_{*}\right) \backslash \mathbf{B}_{r_{*}}\left(x_{*}\right)\right)} \leq e^{-\frac{\alpha_{0}}{h}} .
$$

Let $\eta$ such that $2^{m} \eta=\eta_{0}$ (see (18)) where $m$ will be fixed later (notice that $\eta \leq \eta_{*}$ ). Equation (16) rewrites $\operatorname{div}\left(e^{-\frac{2}{h} f} \nabla \mathrm{q}_{x_{*}}\right)=0$ on $\Omega \backslash \mathrm{B}_{r_{h}}\left(x_{*}\right)$. Therefore, using (20), the Green formula, $\mathrm{q}_{x_{*}} \leq 1$, and $\mathrm{q}_{x_{*}}=0$ on $\partial \mathrm{B}_{r_{h}}\left(x_{*}\right)$, there exist $C>0$ and $\gamma>0$ such that,

$$
\int_{\mathbf{C}_{\eta / 2}\left(x_{*}\right) \backslash \mathrm{B}_{r_{h}}\left(x_{*}\right)}\left|\nabla \mathbf{q}_{x_{*}}\right|^{2} e^{-\frac{2}{h} f}=\int_{\partial \mathbf{C}_{\eta / 2}\left(x_{*}\right) \backslash \mathbf{B}_{r_{h}}\left(x_{*}\right)} \mathbf{q}_{x_{*}} e^{-\frac{2}{h} f} \partial_{\mathbf{n}} \mathbf{q}_{x_{*}} \leq \frac{C}{h^{\gamma}} e^{-\frac{2}{h}\left(\lambda\left(x_{*}\right)-\frac{\eta}{2}\right)},
$$

since $\partial \mathrm{C}_{\eta / 2}\left(x_{*}\right) \subset\left\{f=\lambda\left(x_{*}\right)-\frac{\eta}{2}\right\}$. In addition, since $\mathrm{C}_{\eta}\left(x_{*}\right) \backslash \mathrm{B}_{r_{h}}\left(x_{*}\right) \subset \mathrm{C}_{\eta / 2}\left(x_{*}\right) \backslash \mathrm{B}_{r_{h}}\left(x_{*}\right)$ it holds,

$$
e^{-\frac{2}{h}\left(\lambda\left(x_{*}\right)-\eta\right)} \int_{\mathrm{C}_{\eta}\left(x_{*}\right) \backslash \mathrm{B}_{r_{h}}\left(x_{*}\right)}\left|\nabla \mathbf{q}_{x_{*}}\right|^{2} \leq \int_{\mathrm{C}_{\eta}\left(x_{*}\right) \backslash \mathrm{B}_{r_{h}}\left(x_{*}\right)}\left|\nabla \mathbf{q}_{x_{*}}\right|^{2} e^{-\frac{2}{h} f} \leq \frac{C}{h^{\gamma}} e^{-\frac{2}{h}\left(\lambda\left(x_{*}\right)-\frac{\eta}{2}\right)} .
$$


Therefore, using in addition (19), there exists $\beta>0$ such that for $h$ small enough,

$$
\int_{\mathbf{C}_{\eta}\left(x_{*}\right) \backslash \mathrm{B}_{r_{1}}\left(x_{*}\right)}\left|\nabla \mathbf{q}_{x_{*}}\right|^{2} \leq \int_{\mathbf{C}_{\eta}\left(x_{*}\right) \backslash \mathrm{B}_{r_{h}}\left(x_{*}\right)}\left|\nabla \mathbf{q}_{x_{*}}\right|^{2} \leq \frac{C}{h^{\gamma}} e^{-\frac{\eta}{h}} \leq C e^{-\frac{\beta}{h}}
$$

Thus, from (16), we deduce that $h$ small enough, $\left\|\Delta \mathbf{q}_{x_{*}}\right\|_{L^{2}\left(\mathrm{C}_{\eta}\left(x_{*}\right) \backslash \mathrm{B}_{r_{1}}\left(x_{*}\right)\right)} \leq C e^{-\frac{\beta}{2 h}}$. In the following, $\beta>0$ is a constant which may change from one occurrence to another and does not depend on $h$. Let $\chi_{1} \in \mathcal{C}_{c}^{\infty}\left(\mathrm{C}_{\eta}\left(x_{*}\right) \backslash \mathrm{B}_{r_{1}}\left(x_{*}\right),[0,1]\right)$ be such that $\chi_{1} \equiv 1$ on $\mathrm{C}_{*, 2 \eta} \backslash$ $\mathrm{B}_{r_{2}}\left(x_{*}\right)$. Since $\Delta\left(\chi_{1} \mathbf{q}_{x_{*}}\right)=\chi_{1} \Delta \mathbf{q}_{x_{*}}+\mathrm{q}_{x_{*}} \Delta \chi_{1}+2 \nabla \chi_{1} \cdot \nabla \mathbf{q}_{x_{*}}$, there exists $C$, such that $\left\|\Delta\left(\chi_{1} \mathrm{q}_{x_{*}}\right)\right\|_{L^{2}\left(\mathrm{C}_{\eta}\left(x_{*}\right) \backslash \mathrm{B}_{r_{1}}\left(x_{*}\right)\right)} \leq C$ for $h$ small enough. From the $H^{2}$-elliptic regularity estimate on $\mathrm{C}_{\eta}\left(x_{*}\right) \backslash \mathrm{B}_{r_{1}}\left(x_{*}\right)$, one has:

$$
\left\|\mathrm{q}_{x_{*}}\right\|_{H^{2}\left(\mathrm{C}_{2 \eta}\left(x_{*}\right) \backslash \mathrm{B}_{r_{2}}\left(x_{*}\right)\right)} \leq C .
$$

Let $\alpha \in(0,1)$ be an irrational number such that $p_{1}=\frac{2 d}{d-2 \alpha}>0$. From the Gagliardo-Nirenberg interpolation inequality (see 44, Lecture II]), the following inequality holds:

$$
\begin{aligned}
C\left\|\nabla \mathbf{q}_{x_{*}}\right\|_{L^{p_{1}}\left(\mathrm{C}_{2 \eta}\left(x_{*}\right) \backslash \mathrm{B}_{r_{2}}\left(x_{*}\right)\right) \leq} & \left\|\mathbf{q}_{x_{*}}\right\|_{H^{2}\left(\mathrm{C}_{2 \eta}\left(x_{*}\right) \backslash \mathrm{B}_{r_{2}}\left(x_{*}\right)\right)}^{\alpha}\left\|\nabla \mathbf{q}_{x_{*}}\right\|_{L^{2}\left(\mathrm{C}_{2 \eta}\left(x_{*}\right) \backslash \mathrm{B}_{r_{2}}\left(x_{*}\right)\right)}^{1-\alpha} \\
& +\left\|\nabla \mathbf{q}_{x_{*}}\right\|_{L^{2}\left(\mathrm{C}_{2 \eta}\left(x_{*}\right) \backslash \mathrm{B}_{r_{2}}\left(x_{*}\right)\right)} \leq e^{-\frac{\beta}{h}} .
\end{aligned}
$$

Then, from (16), one deduces that $\left\|\Delta \mathbf{q}_{x_{*}}\right\|_{L^{p_{1}}\left(\mathrm{C}_{2 \eta}\left(x_{*}\right) \backslash \mathrm{B}_{r_{2}}\left(x_{*}\right)\right)} \leq C e^{-\frac{\beta}{h}}$. Using a cutoff function $\chi_{2} \in \mathcal{C}_{c}^{\infty}\left(\mathrm{C}_{2 \eta}\left(x_{*}\right) \backslash \mathrm{B}_{r_{2}}\left(x_{*}\right),[0,1]\right)$ such that $\chi_{2} \equiv 1$ on $\mathrm{C}_{4 \eta}\left(x_{*}\right) \backslash \mathrm{B}_{r_{3}}\left(x_{*}\right)$, we get from the $W^{2, p_{1}}$-elliptic regularity estimate, that $\left\|\mathrm{q}_{x_{*}}\right\|_{W^{2, p_{1}}\left(\mathrm{C}_{4 \eta}\left(x_{*}\right) \backslash \mathrm{B}_{r_{3}}\left(x_{*}\right)\right)} \leq C$. Let $p_{2}=\frac{2 d}{d-4 \alpha}$, from the Gagliardo-Nirenberg interpolation inequality (see [44, Lecture II]), one obtains:

$$
\begin{aligned}
C\left\|\nabla \mathbf{q}_{x_{*}}\right\|_{L^{p_{2}}\left(\mathrm{C}_{4 \eta}\left(x_{*}\right) \backslash \mathrm{B}_{r_{3}}\left(x_{*}\right)\right) \leq} & \left\|\mathbf{q}_{x_{*}}\right\|_{W^{2, p_{1}}\left(\mathrm{C}_{4 \eta}\left(x_{*}\right) \backslash \mathrm{B}_{r_{3}}\left(x_{*}\right)\right)}^{\alpha}\left\|\nabla \mathbf{q}_{x_{*}}\right\|_{L^{p_{1}}\left(\mathrm{C}_{4 \eta}\left(x_{*}\right) \backslash \mathrm{B}_{r_{3}}\left(x_{*}\right)\right)}^{1-\alpha} \\
& +\left\|\nabla \mathbf{q}_{x_{*}}\right\|_{L^{p_{1}}\left(\mathrm{C}_{4 \eta}\left(x_{*}\right) \backslash \mathrm{B}_{r_{3}}\left(x_{*}\right)\right)} \leq e^{-\frac{\beta}{h}}
\end{aligned}
$$

We repeat this procedure $m-2$ times such that $d-2 m \alpha \leq 0$, the Galgliardo-Nirenberg interpolation inequality implies that $\left\|\nabla \mathrm{q}_{x_{*}}\right\|_{L^{\infty}\left(\mathrm{C}_{2} m_{\eta}\left(x_{*}\right) \backslash \mathrm{B}_{r_{m+1}}\left(x_{*}\right)\right)} \leq C e^{-\frac{\beta}{h}}$ which ends the proof of (25) since $\eta_{0}=2^{m} \eta$ and $r_{m+1}=r_{*}$ (see (18)).

Step 3: Let $r_{* *}>r_{*}$ such that $\mathrm{B}_{r_{* *}}\left(x_{*}\right) \subset \mathrm{U}$ (see (17)). From Lemma 4 , for $h$ small enough, it holds:

$$
\max _{y \in \mathrm{B}_{r_{* *}}\left(x_{*}\right)}\left|\mathbf{p}_{x_{*}}(y)-1\right|=O\left(e^{-\frac{c}{h}}\right) .
$$

When the dimension $d \geq 2$, the open set $\mathrm{C}_{\eta}\left(x_{*}\right) \backslash \mathrm{B}_{r_{*}}\left(x_{*}\right)$ is path-connected. Indeed $\mathrm{B}_{r_{*}}\left(x_{*}\right)$ is a closed ball included in the open connected set $C_{\eta}\left(x_{*}\right)$ (see (17) and 24) and thus, when $d \geq 2$, $\mathrm{C}_{\eta}\left(x_{*}\right) \backslash \mathrm{B}_{r_{*}}\left(x_{*}\right)$ is connected. Since it is moreover locally path-connected, $\mathrm{C}_{\eta}\left(x_{*}\right) \backslash \mathrm{B}_{r_{*}}\left(x_{*}\right)$ is path-connected. When $d=1, \mathrm{C}_{\eta}\left(x_{*}\right) \backslash \mathrm{B}_{r_{*}}\left(x_{*}\right)$ is the disjoint union of two open intervals $\mathrm{I}_{1}$ and $\mathrm{I}_{2}$. Let $z_{* *} \in \partial \mathrm{B}_{r_{* *}}\left(x_{*}\right)$. Then, when $d \geq 2$ for $\eta>0$ small enough and for all $y \in \mathrm{C}_{\eta}\left(x_{*}\right) \backslash \mathrm{B}_{r_{*}}\left(x_{*}\right)$, considering a smooth curve $\gamma:[0,1] \rightarrow \mathrm{C}_{\eta}\left(x_{*}\right) \backslash \mathrm{B}_{r_{*}}\left(x_{*}\right)$ such that $\gamma(0)=y$ and $\gamma(1)=z$ (because $\mathrm{C}_{\eta}\left(x_{*}\right) \backslash \mathrm{B}_{r_{*}}\left(x_{*}\right)$ is open, path-connected, and locally smooth), it follows from Equation 25 that there exists $\beta>0$ such that for $h$ small enough:

$$
\sup _{y \in \mathrm{C}_{\eta}\left(x_{*}\right) \backslash \mathrm{B}_{r_{*}}\left(x_{*}\right)}\left|\mathrm{p}_{x_{*}}(y)-\mathrm{p}_{x_{*}}\left(z_{* *}\right)\right| \leq e^{-\frac{\beta}{h}} .
$$

Let us mention that when $d=1$, the previous estimate also holds by choosing $z_{* *} \in \mathrm{I}_{k}$ if $y \in \mathrm{I}_{k}$ $(k \in\{1,2\})$. Using in addition (26), for $\eta>0$ small enough, there exists $c>0$, for $h$ small enough, one has:

$$
\mathrm{p}_{x_{*}}(y)=1+O\left(e^{-\frac{c}{h}}\right) \text {, uniformly on } y \in \mathrm{C}_{\eta}\left(x_{*}\right) .
$$


This concludes the proof of Proposition 5 when $\mathrm{K} \subset \mathrm{C}\left(x_{*}\right)$ (indeed in this case there exists $\eta>0$ such that $\left.\mathrm{K} \subset \mathrm{C}_{\eta}\left(x_{*}\right)\right)$.

Step 4: Let us consider $\mathrm{K} \subset \mathcal{A}_{\Omega}\left(\mathrm{C}\left(x_{*}\right)\right)$. Since $\mathrm{C}\left(x_{*}\right) \subset \mathcal{A}_{\Omega}\left(\mathrm{C}\left(x_{*}\right)\right)$ and $\mathrm{C}\left(x_{*}\right)$ is open, there exists $T_{\mathrm{K}} \geq 0$ such that for all $x \in \mathrm{K}$,

$$
\varphi_{T_{\mathrm{K}}}(x) \in \mathrm{C}\left(x_{*}\right)
$$

where we recall $\varphi_{t}(x)$ is defined by (4). Moreover, since $\mathrm{K}$ is a compact subset of $\mathcal{A}_{\Omega}\left(\mathrm{C}\left(x_{*}\right)\right)$ and for all $x \in \mathrm{K}, t_{x}=+\infty$ (i.e. $\varphi_{t}(x) \in \Omega$ for all $t \geq 0$ ), the set $\left\{\varphi_{t}(x), t \in\left[0, T_{K}\right]\right.$ and $\left.x \in \mathrm{K}\right\}$ is a compact subset of $\Omega$. Therefore, there exists $\delta>0$ such that all continuous curves $\gamma:\left[0, T_{\mathrm{K}}\right] \rightarrow \bar{\Omega}$ such that

$$
\exists x \in \mathrm{K}, \sup _{t \in\left[0, T_{\mathrm{K}}\right]}\left|\gamma(t)-\varphi_{t}(x)\right| \leq \delta
$$

satisfy:

$$
\forall t \in\left[0, T_{\mathrm{K}}\right], \gamma(t) \in \Omega .
$$

Moreover, up to choosing $\delta>0$ smaller, there exists $\alpha_{\mathrm{K}}>0$ such that

$$
\left\{\varphi_{T_{\mathrm{K}}}(x)+z, x \in \mathrm{K} \text { and }|z| \leq \delta\right\} \subset \mathrm{C}_{\alpha_{\mathrm{K}}}\left(x_{*}\right) .
$$

Let us notice that when $X_{0}=x \in \mathrm{K}$ and $\sup _{t \in\left[0, T_{\mathrm{K}}\right]}\left|X_{t}-\varphi_{t}(x)\right| \leq \delta$, it holds from 29) and (30):

$$
\tau_{\Omega}>T_{\mathrm{K}} \text { and } X_{T_{\mathrm{K}}} \in \mathrm{C}_{\alpha_{\mathrm{K}}}\left(x_{*}\right)
$$

Let us recall the following estimate of Freidlin and Wentzell (see 17, Theorems 2.2 and 2.3 in Chapter 3, and Theorem 1.1 in Chapter 4] and [9]). For all $x \in \mathrm{K}$, it holds:

$$
\limsup _{h \rightarrow 0} h \ln \mathbb{P}_{x}\left[\sup _{t \in\left[0, T_{\mathrm{K}}\right]}\left|X_{t}-\varphi_{t}(x)\right| \geq \delta\right] \leq-I_{x, T_{\mathrm{K}}}
$$

where

$$
I_{x, T_{\mathrm{K}}}=\frac{1}{2} \inf _{\gamma \in H_{x, T_{\mathrm{K}}}^{1}(\delta)} \int_{0}^{T_{\mathrm{K}}}\left|\frac{d}{d t} \gamma(t)+\nabla f(\gamma(t))\right|^{2} d t \in \mathbb{R}_{+}^{*} \cup\{+\infty\},
$$

and $H_{x, T_{\mathrm{K}}}^{1}(\delta)$ is the set of curves $\gamma:\left[0, T_{\mathrm{K}}\right] \rightarrow \Omega$ of regularity $H^{1}$ such that $\gamma(0)=x$ and $\sup _{t \in\left[0, T_{\mathrm{K}}\right]}\left|\gamma(t)-\varphi_{t}(x)\right| \geq \delta$. The functional $I_{x, T_{\mathrm{K}}}$ is the quasi-potential of the process (1) on the interval time $\left[0, T_{\mathrm{K}}\right]$ when $X_{0}=x$. Since $\mathrm{K}$ is compact, there exists $\eta_{\mathrm{K}}>0$ such that for $h$ small enough, it holds:

$$
\sup _{x \in \mathrm{K}} \mathbb{P}_{x}\left[\sup _{t \in\left[0, T_{\mathrm{K}}\right]}\left|X_{t}-\varphi_{t}(x)\right| \geq \delta\right] \leq e^{-\frac{\eta_{\mathrm{K}}}{h}}
$$

Let us now concludes the proof of Proposition 5. For $x \in \mathrm{K}$, one writes $\mathrm{p}_{x_{*}}(x)=a(x)+b(x)$, where

$$
a(x)=\mathbb{P}_{x}\left[\left\{\tau_{\mathrm{B}_{r_{h}}\left(x_{*}\right)}<\tau_{\Omega^{c}}\right\} \cap\left\{\sup _{t \in\left[0, T_{\mathrm{K}}\right]}\left|X_{t}-\varphi_{t}(x)\right|<\delta\right\}\right]
$$

and

$$
b(x)=\mathbb{P}_{x}\left[\left\{\tau_{\mathrm{B}_{r_{h}}\left(x_{*}\right)}<\tau_{\Omega^{c}}\right\} \cap\left\{\sup _{t \in\left[0, T_{\mathrm{K}}\right]}\left|X_{t}-\varphi_{t}(x)\right| \geq \delta\right\}\right]
$$


Using (33), it holds for $h$ small enough: $\max _{x \in \mathrm{K}} b(x) \leq e^{-\frac{\eta_{K}}{h}}$. Using (31), 27) with $C_{\alpha_{K}}\left(x_{*}\right)$, (33), and the Markov property of the process (1), there exists $c>0$ such that for all $x \in \mathrm{K}$, one has when $h \rightarrow 0$ :

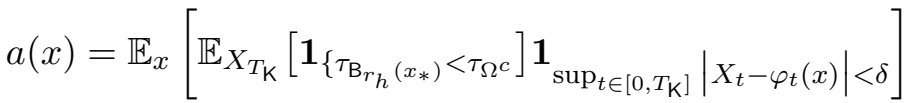

$$
\begin{aligned}
& =\left(1+O\left(e^{-\frac{c}{h}}\right)\right) \times \mathbb{P}_{x}\left[\sup _{t \in\left[0, T_{\mathrm{K}}\right]}\left|X_{t}-\varphi_{t}(x)\right| \leq \delta\right]=1+O\left(e^{-\frac{c}{h}}\right) \text {, uniformly in } x \in \mathrm{K} .
\end{aligned}
$$

Hence, there exists $c>0$ such that for all $x \in \mathrm{K}$, one has when $h \rightarrow 0: \mathrm{p}_{x_{*}}(x)=1+O\left(e^{-\frac{c}{h}}\right)$, uniformly in $x \in \mathrm{K}$. This concludes the proof of Proposition 5

We will also need the slightly different version of Proposition 5 .

Proposition 6. Assume that $f: \bar{\Omega} \rightarrow \mathbb{R}$ is a $\mathcal{C}^{\infty}$ Morse function. Let $x_{*}$ be a local minimum of $f$ in $\Omega$. Consider a compact subset $\mathrm{K}$ of $\mathcal{A}_{\Omega}\left(\mathrm{C}\left(x_{*}\right)\right)$. Let $T_{\mathrm{K}}$ be such that 28$)$ holds. Then, there exist $\delta_{\mathrm{K}}>0, \alpha_{\mathrm{K}}>0$, and a smooth domain $\Omega_{\mathrm{K}}$ containing $\mathrm{K}$ with $\overline{\Omega_{\mathrm{K}}} \subset \Omega$, such that all continuous curves $\gamma:\left[0, T_{\mathrm{K}}\right] \rightarrow \bar{\Omega}$ such that

$$
\exists x \in \mathrm{K}, \sup _{t \in\left[0, T_{\mathrm{K}}\right]}\left|\gamma(t)-\varphi_{t}(x)\right| \leq \delta_{\mathrm{K}} \quad(\text { see }(4)),
$$

satisfy:

1. $\forall t \in\left[0, T_{\mathrm{K}}\right], \gamma(t) \in \Omega_{\mathrm{K}}$.

2. $\left\{\varphi_{T_{\mathrm{K}}}(x)+z, x \in \mathrm{K}\right.$ and $\left.|z| \leq \delta_{\mathrm{K}}\right\} \subset \mathrm{C}_{\alpha_{\mathrm{K}}}\left(x_{*}\right)$ (see $(22)$ ), and $\overline{\mathrm{C}_{\alpha_{\mathrm{K}}}\left(x_{*}\right)} \subset \Omega_{\mathrm{K}}$.

Moreover, there exist $h_{0}>0$ and $c>0$ such that for all $h \in\left(0, h_{0}\right)$, it holds:

$$
\max _{y \in \mathrm{K}}\left|\mathbb{P}_{y}\left[\tau_{\mathrm{B}_{r_{h}}\left(x_{*}\right)}<\tau_{\Omega_{\mathrm{K}}^{c}}\right]-1\right| \leq e^{-\frac{c}{h}}
$$

Proof. Let us first prove items 1 and 2 in Proposition 6. Let $\mathrm{K} \subset \mathcal{A}_{\Omega}\left(\mathrm{C}\left(x_{*}\right)\right)$ and $T_{\mathrm{K}}$ be such that (28) holds. The set $\left\{\varphi_{t}(x), t \in\left[0, T_{\mathrm{K}}\right]\right.$ and $\left.x \in \mathrm{K}\right\}$ is a compact subset of $\Omega$ and the set $\left\{\varphi_{T_{\mathrm{K}}}(x)+z, x \in \mathrm{K}\right.$ and $\left.|z| \leq \delta\right\}$ is a compact subset of $\mathrm{C}\left(x_{*}\right)$. Thus, for $\delta>0$ small enough, the compact set $\mathrm{M}:=\left\{y \in \bar{\Omega}, \exists x \in \mathrm{K}\right.$ and $t \in\left[0, T_{\mathrm{K}}\right]$ such that $\left.\left|y-\varphi_{t}(x)\right| \leq \delta\right\}$ is included in $\Omega$, and $\left\{\varphi_{T_{\mathrm{K}}}(x)+z, x \in \mathrm{K}\right.$ and $\left.|z| \leq \delta\right\}$ is included in $\mathrm{C}_{\alpha_{\mathrm{K}}}\left(x_{*}\right)$ for some $\alpha_{\mathrm{K}}>0$ small enough. Because $\mathrm{K}, \mathrm{M}$, and $\overline{\mathrm{C}_{\alpha_{\mathrm{K}}}\left(x_{*}\right)}$ are compact subsets of $\Omega$, there exists a smooth domain $\Omega_{\mathrm{K}}$ such that $\mathrm{K} \cup \mathrm{M} \cup \overline{\mathrm{C}_{\alpha_{\mathrm{K}}}\left(x_{*}\right)} \subset \Omega_{\mathrm{K}}$ and $\overline{\Omega_{\mathrm{K}}} \subset \Omega$. This proves items 1 and 2 in Proposition 6. Up to choosing $\Omega_{\mathrm{K}}$ larger, let us assume that $\alpha_{\mathrm{K}} \in\left(0, \lambda\left(x_{*}\right)-c_{*, M}\right)$, see (23). Let us now prove Equation (34). The proof of (34) is the same as the one made to prove Proposition 5. For the sake of completeness, we recall the main arguments. Let us denote by $\tilde{\mathrm{p}}_{x_{*}}: y \in \overline{\Omega_{\mathrm{K}}} \mapsto \mathbb{P}_{y}\left[\tau_{\mathrm{B}_{r_{h}}\left(x_{*}\right)}<\tau_{\Omega_{\mathrm{K}}^{c}}\right]$. Since $\alpha_{\mathrm{K}} \in\left(0, \lambda\left(x_{*}\right)-c_{*, M}\right)$, there exists $\varepsilon_{0}>0$ such that for all $\varepsilon \in\left(0, \varepsilon_{0}\right), C_{\alpha_{K}+\varepsilon}$ is a $\mathcal{C}^{\infty}$ open connected component of $\left\{f<\lambda\left(x_{*}\right)-\alpha_{K}-\varepsilon\right\}$ which is included in $\Omega_{\mathrm{K}}$ (see the lines after (24)). Thus, with the same arguments as those used to prove (27) above, one obtains that for all $\varepsilon \in\left(0, \varepsilon_{0}\right)$, there exists $c>0$ such that for $h$ small enough:

$$
\tilde{\mathbf{p}}_{x_{*}}(y)=1+O\left(e^{-\frac{c}{h}}\right), \quad \text { uniformly on } y \in \mathrm{C}_{\alpha_{\mathrm{K}}+\varepsilon}\left(x_{*}\right) .
$$


Since $\left\{\varphi_{T_{\mathrm{K}}}(x)+z, x \in \mathrm{K}\right.$ and $\left.|z| \leq \delta\right\}$ is a compact subset of the open set $\mathrm{C}_{\alpha_{\mathrm{K}}}\left(x_{*}\right)$, for $\varepsilon \in\left(0, \varepsilon_{0}\right)$ small enough, it holds

$$
\left\{\varphi_{T_{\mathrm{K}}}(x)+z, x \in \mathrm{K} \text { and }|z| \leq \delta\right\} \subset \mathrm{C}_{\alpha_{\mathrm{K}}+\varepsilon}\left(x_{*}\right) .
$$

From item 1 in Proposition 6, when $X_{0}=x \in \mathrm{K}$ and $\sup _{t \in\left[0, T_{\mathrm{K}}\right]}\left|X_{t}-\varphi_{t}(x)\right| \leq \delta$, it holds:

$$
\tau_{\Omega_{\mathrm{K}}^{c}}>T_{\mathrm{K}} \text {, and from }(36), X_{T_{\mathrm{K}}} \in \mathrm{C}_{\alpha_{\mathrm{K}}+\varepsilon}\left(x_{*}\right) \text {. }
$$

One then writes for all $x \in \mathrm{K}, \tilde{\mathrm{p}}_{x_{*}}(x)=c(x)+d(x)$, where $c(x)=\mathbb{P}_{x}\left[\left\{\tau_{\mathrm{B}_{r_{h}}\left(x_{*}\right)}<\tau_{\Omega_{\mathrm{K}}^{c}}\right\} \cap\right.$ $\left.\left\{\sup _{t \in\left[0, T_{\mathrm{K}}\right]}\left|X_{t}-\varphi_{t}(x)\right|<\delta\right\}\right]$ and $d(x)=\mathbb{P}_{x}\left[\left\{\tau_{\mathrm{B}_{h}\left(x_{*}\right)}<\tau_{\Omega_{\mathrm{K}}^{c}}\right\} \cap\left\{\sup _{t \in\left[0, T_{\mathrm{K}}\right]}\left|X_{t}-\varphi_{t}(x)\right| \geq \delta\right\}\right]$. Using (33), it holds for $h$ small enough: $\sup _{x \in \mathrm{K}} d(x) \leq e^{-\frac{c}{h}}$, for some $c>0$ independent of $h$. The Markov property together with (35) and (37), imply that there exists $c>0$ such that when $h \rightarrow 0, c(x)=1+O\left(e^{-\frac{c}{h}}\right)$, uniformly in $x \in \mathrm{K}$. This proves (34) and concludes the proof of Proposition 6.

We will also need a leveling result of $\mathrm{p}_{x_{*}}$ in $\mathrm{C} \subset \mathcal{C}$ (see $(7)$ ) when $\mathrm{C} \neq \mathrm{C}(x)$. This leveling result is the purpose of Proposition 8 below which is based on an estimate on the most probable places of exit from a bounded domain when $\mathrm{N}=1$, see the next section.

\subsection{The case when $\mathrm{N}=1$}

In this section, we prove the following result.

Proposition 7. Let $f: \bar{\Omega} \rightarrow \mathbb{R}$ be a $\mathcal{C}^{\infty}$ Morse function such that (H1) is satisfied with $\mathrm{N}=1$ (i.e. $\left\{f<\min _{\partial \Omega} f\right\}$ is connected). Let $\mathrm{K}$ be compact subset of $\mathcal{A}_{\Omega}\left(\left\{f<\min _{\partial \Omega} f\right\}\right)$ (see (5)) and $\Gamma$ be an open subset of $\partial \Omega$ such that $\min _{\bar{\Gamma}} f>\min _{\partial \Omega} f$. Then,

$$
\text { there exists } c>0 \text { s.t. for } h \text { small enough: } \max _{x \in \mathrm{K}} \mathbb{P}_{x}\left[X_{\tau_{\Omega^{c}}} \in \Gamma\right] \leq e^{-\frac{c}{h}} \text {. }
$$

In Corollary 10 below, we will generalize $(38)$ to more general settings (e.g. when $\mathrm{N}>1$ ).

Before going through the proof of Proposition 7, we recall the notion of separating saddle points of $f$ in $\Omega$. A point $z \in \Omega$ is a separating saddle point of $f$ in $\Omega$ if it is a saddle point of $f$ and for $r>0$ small enough, $\mathrm{B}_{r}(z) \cap\{f<f(z)\}$ has two connected components which are included in two different connected components of $\{f<f(z)\}$. The notion of separating saddle points was first introduced in [22] in the boundaryless case and then adapted to the boundary case in [14, 30].

Proof. Let $f: \bar{\Omega} \rightarrow \mathbb{R}$ be a $\mathcal{C}^{\infty}$ function. Before starting the proof of Proposition 7 , we introduce some preliminary results we will need. Let us choose an eigenfunction $u_{1, h}$ associated with the principal eigenvalue $\lambda_{1, h}$ of $\mathcal{L}_{f, h}^{\text {Dir }}$ such that

$$
u_{1, h}>0 \text { on } \Omega \text { and } \int_{\Omega} u_{1, h}^{2} e^{-\frac{2}{h} f}=1 .
$$

For ease of notation, we write $L_{w}^{2}(\Omega)$ (resp. $L^{2}(\Omega)$ ) for $L^{2}\left(\Omega, e^{-\frac{2}{h} f} d x\right)$ (resp. for $L^{2}(\Omega, d x)$ ). The subscript $w$ stands for the fact that we consider the weight function $e^{-\frac{2}{h} f}$ in the scalar product. The proof of Proposition 7 is based on the use of the quasi-stationary distribution of 
the process (1) in $\Omega$ : we will indeed first prove (38) when $X_{0}$ is distributed according to this distribution, and then, with the help of a leveling result, we will derive it when $X_{0}=x \in \mathrm{K}$. A quasi-stationary distribution for the process (1) in $\Omega$ is a probability measure $\mu$ supported in $\Omega$ such that for all measurable sets $A \subset \Omega$ and for all $t \geq 0$,

$$
\mu(A)=\frac{\int_{\Omega} \mathbb{P}_{x}\left[X_{t} \in A, t<\tau_{\Omega}\right] \mu(d x)}{\int_{\Omega} \mathbb{P}_{x}\left[t<\tau_{\Omega^{c}}\right] \mu(d x)} .
$$

Let us recall that (see for example [27]) the unique quasi-stationary distribution $\nu_{h}$ of the dynamics (1) in $\Omega$ is the measure

$$
\nu_{h}(d x)=\frac{u_{1, h}(x) e^{-\frac{2}{h} f(x)}}{\int_{\Omega} u_{1, h} e^{-\frac{2}{h} f}} d x .
$$

Moreover, according to [27], when $X_{0} \sim \nu_{h}, X_{\tau_{\Omega^{c}}}$ has a density with respect to the Lebesgue measure on $\partial \Omega$ which is given by:

$$
z \in \partial \Omega \mapsto-\frac{h}{2 \lambda_{1, h}} \frac{\partial_{\mathrm{n}} u_{1, h}(z) e^{-\frac{2}{h} f(z)}}{\int_{\Omega} u_{1, h} e^{-\frac{2}{h} f}} .
$$

Let us now assume that $f: \bar{\Omega} \rightarrow \mathbb{R}$ is a Morse function. From the analysis led in the proof of 30 , Theorem 1] together with (3), for any $L_{w}^{2}(\Omega)$-normalized eigenfunction $w_{h}$ of $\mathcal{L}_{f, h}^{\text {Dir }}$ associated with an eigenvalue of order $o(1)$ when $h \rightarrow 0$, it holds, for every neighborhood $\mathbf{W}$ of the local minima of $f$ in $\Omega$ :

$$
\lim _{h \rightarrow 0}\left\|w_{h} \mathbf{1}_{\mathrm{W}}\right\|_{L_{w}^{2}(\Omega)}=1 .
$$

Let us now assume that (H1) is satisfied with $\mathbf{N}=1$. Let us define $c_{M}=\max \{f(x), x \in\{f<$ $\left.\min _{\partial \Omega} f\right\}$ such that $\left.|\nabla f(x)|=0\right\}<\min _{\partial \Omega} f$. In the following, for $\alpha>0, \mathrm{C}_{\alpha}$ denotes the open set $\left\{f<\min _{\partial \Omega} f-\alpha\right\}$. Let us recall that for $\alpha<\min _{\partial \Omega} f-c_{M}, \mathrm{C}_{\alpha}$ is a smooth connected open subset of $\Omega$ (see indeed the lines after $(22)$ ). Moreover, for any $c_{0}>0$, we denote by $V_{0}$ a smooth open connected subset of $\Omega$ such that $\overline{\left\{f<\min _{\partial \Omega} f\right\}} \subset \overline{V_{0}}$ and $f \leq \min _{\partial \Omega} f+c_{0}$ on $\mathrm{V}_{0}$. Since $\partial\left\{f<\min _{\partial \Omega} f\right\} \cap \partial \Omega \neq \emptyset$, we choose $\mathrm{V}_{0}$ such that $\partial \mathrm{V}_{0} \cap \partial \Omega$ has a non zero Lebesgue measure (in $\partial \Omega$ ). We are now ready to prove Proposition 7. The proof is divided into several steps.

Step 1: Let us prove that:

$$
\lim _{h \rightarrow 0} h \log \lambda_{1, h}=-2\left(\min _{\partial \Omega} f-\min _{\bar{\Omega}} f\right) \text { and } \liminf _{h \rightarrow 0} h \log \lambda_{2, h} \geq-2\left(c_{M}-\min _{\bar{\Omega}} f\right) .
$$

When (H2) holds, 43) is a consequence of [30, Theorem 2] and (3). Moreover, under (H1), the set $\partial\left\{f<\min _{\partial \Omega} f\right\} \cap \Omega$ does not contain separating saddle point of $f$ in $\Omega$. Thus, if $\overline{(\mathbf{H 2})}$ holds, according to (3) and [30, Theorem 3] (with $\mathrm{m}^{*}=1$ and $\mathrm{C}_{\mathbf{j}}\left(x_{1}\right)=\left\{f<\min _{\partial \Omega} f\right\}$ there), one has more precisely when $h \rightarrow 0$ :

$$
\lambda_{1, h}=\left(\Theta_{1,1} h^{-\frac{1}{2}}(1+O(h))+\Theta_{1,2}(1+O(\sqrt{h}))\right) e^{-\frac{2}{h}\left(\min _{\partial \Omega} f-\min _{\bar{\Omega}} f\right)},
$$

where $\Theta_{1,1}$ and $\Theta_{1,2}$ are defined at the beginning of Section 1.4 (recall that $C_{1}=\left\{f<\min _{\partial \Omega} f\right\}$ because we assume here that $\mathrm{N}=1)$. Actually, we will prove $(43)$ and $(38)$ without assuming (H2). Let us first prove the first limit in 43). Let $\chi \in \mathcal{C}_{c}^{\infty}\left(\left\{f<\min _{\partial \Omega} f\right\}\right)$ be such that 
$\chi=1$ on $\mathrm{C}_{\alpha}$, for $\alpha>0$. Then, it follows from a Laplace's method that for all $\alpha>0$ small enough such that $\arg \min _{\bar{\Omega}} f \subset \mathrm{C}_{\alpha}$, there exists $C>0$ such that for $h$ small enough:

$$
\lambda_{1, h} \leq\left\langle\frac{\mathcal{L}_{f, h} \chi}{\|\chi\|_{L_{w}^{2}(\Omega)}}, \frac{\chi}{\|\chi\|_{L_{w}^{2}(\Omega)}}\right\rangle_{L_{w}^{2}(\Omega)}=\frac{h}{2} \frac{\|\nabla \chi\|_{L_{w}^{2}(\Omega)}^{2}}{\|\chi\|_{L_{w}^{2}(\Omega)}^{2}} \leq C h^{-\frac{d}{2}+1} e^{-\frac{2}{h}\left(\min _{\partial \Omega} f-\min _{\bar{\Omega}} f-\alpha\right)} .
$$

Because $\alpha>0$ is arbitrary, this proves that $\lim _{h \rightarrow 0} h \log \lambda_{1, h} \leq-2\left(\min _{\partial \Omega} f-\min _{\bar{\Omega}} f\right)$. To prove the reverse inequality, inspired by [33, Lemma 5.9], let us argue by contradiction: assume that there exists $c>0$ and a sequence $\left(h_{n}\right)_{n \in \mathbb{N}}$ such that $\lim _{n \rightarrow+\infty} h_{n}=0$ and for all $n \geq 0$, $\lambda_{1, h_{n}} \leq e^{-\frac{2}{h_{n}}\left(\min _{\partial \Omega} f-\min _{\bar{\Omega}} f+c\right)}$. Let $c_{0}<c$. From (39) and since $f \leq \min _{\partial \Omega} f+c_{0}$ on $\mathrm{V}_{0}$, it holds:

$$
\frac{h_{n} e^{-\frac{2}{h_{n}}\left(\min _{\partial \Omega} f+c_{0}\right)} \int_{\mathrm{V}_{0}}\left|\nabla u_{1, h_{n}}\right|^{2}}{2} \leq \frac{h_{n}}{2} \int_{\mathrm{V}_{0}}\left|\nabla u_{1, h_{n}}\right|^{2} e^{-\frac{2}{h_{n}} f} \leq e^{-\frac{2}{h_{n}}\left(\min _{\partial \Omega} f-\min _{\bar{\Omega}} f+c\right)},
$$

which implies that: $\left\|\nabla\left(u_{1, h_{n}} e^{-\frac{1}{h_{n}} \min _{\bar{\Omega}} f}\right)\right\|_{L^{2}\left(\mathrm{~V}_{0}\right)}^{2} \leq \frac{2}{h_{n}} e^{-\frac{c-c_{0}}{h_{n}}} \rightarrow 0$ as $n \rightarrow \infty$. Defining $c_{n}=$ $\int_{\mathrm{V}_{0}} u_{n} e^{-\frac{1}{h_{n}} \min _{\bar{\Omega}} f}\left(\int_{\mathrm{V}_{0}} 1\right)^{-1}$, the Poincaré-Wirtinger inequality (valid because $\mathrm{V}_{0}$ is a smooth bounded domain) then implies:

$$
\lim _{n \rightarrow+\infty}\left\|u_{n} e^{-\frac{1}{h_{n}} \min _{\bar{\Omega}} f}-c_{n}\right\|_{L^{2}\left(\mathrm{~V}_{0}\right)}=0 \text { and thus, } \lim _{n \rightarrow+\infty}\left\|u_{n} e^{-\frac{1}{h_{n}} \min _{\bar{\Omega}} f}-c_{n}\right\|_{H^{1}\left(\mathrm{~V}_{0}\right)}=0 .
$$

Because $\left.u_{n}\right|_{\partial \mathrm{V}_{0} \cap \partial \Omega}=0$, it follows from the the trace theorem, that $\lim _{n \rightarrow+\infty} c_{n}=0$. Therefore, it holds: $\lim _{n \rightarrow+\infty}\left\|u_{n} e^{-\frac{1}{h_{n}} \min _{\bar{\Omega}} f}\right\|_{L^{2}\left(\mathrm{~V}_{0}\right)}=0$ and thus, $\lim _{n \rightarrow+\infty}\left\|u_{n} e^{-\frac{1}{h_{n}} f}\right\|_{L^{2}\left(\mathrm{~V}_{0}\right)}=0$ which contradicts (42). This concludes the proof of the first limit in (43). Let us now prove the second limit in (43). For that purpose, we argue again by contradiction: assume that there exists a constant $c \in\left(0, \min _{\partial \Omega} f-c_{M}\right)$ and a decreasing sequence $\left(h_{n}\right)_{n \in \mathbb{N}}$ such that $\lim _{n \rightarrow+\infty} h_{n}=0^{+}$ and for all $n \in \mathbb{N}, \lambda_{2, h_{n}} \leq e^{-\frac{2}{h_{n}}\left(c_{M}-\min _{\bar{\Omega}} f+c\right)}$. Then, for $\alpha>0$ small enough (such that $\mathbf{1}_{C_{\alpha}} \neq 0$ ), using the Max-Min principle (see for example [21, Theorem 11.7]), for all $n \in \mathbb{N}$, there exists a $L_{w}^{2}(\Omega)$-normalized function $\varphi_{n} \in D\left(\mathcal{L}_{f, h}^{\text {Dir }}\right)$ such that

$$
\left\langle\varphi_{n}, 1\right\rangle_{L_{w}^{2}\left(\mathrm{C}_{\alpha}\right)}=0 \text { and }\left\langle\mathcal{L}_{f, h_{n}} \varphi_{n}, \varphi_{n}\right\rangle_{L_{w}^{2}(\Omega)}=\frac{h_{n}}{2}\left\|\nabla \varphi_{n}\right\|_{L_{w}^{2}(\Omega)}^{2} \leq e^{-\frac{2}{h_{n}}\left(c_{M}-\min _{\bar{\Omega}} f+c\right)} .
$$

Choosing $\alpha=\min _{\partial \Omega} f-c_{M}-\frac{c}{2}$ and reasoning as in 46), one deduces that: $\left\|\nabla\left(\varphi_{n} e^{-\frac{1}{h_{n}} \min _{\bar{\Omega}} f}\right)\right\|_{L^{2}\left(\mathrm{C}_{\alpha}\right)}^{2} \leq \frac{2}{h_{n}} e^{-\frac{c-c / 2}{h_{n}}} \rightarrow 0$ as $n \rightarrow \infty$. The Poincaré-Wirtinger inequality (valid because $\mathrm{C}_{\alpha}$ is a smooth bounded domain since $\alpha<\min _{\partial \Omega} f-c_{M}$ ), there exists $c_{n} \in \mathbb{R}$ such that $\lim _{n \rightarrow+\infty}\left\|\varphi_{n} e^{-\frac{1}{h_{n}} \min _{\bar{\Omega}} f}-c_{n}\right\|_{L^{2}\left(\mathrm{C}_{\alpha}\right)}=0$. Therefore,

$$
\lim _{n \rightarrow+\infty}\left\|\varphi_{n} e^{-\frac{1}{h_{n}} f}-c_{n} e^{-\frac{1}{h_{n}}\left(f-\min _{\bar{\Omega}} f\right)}\right\|_{L^{2}\left(\mathrm{C}_{\alpha}\right)}=0
$$

Since $\left\langle\varphi_{n}, 1\right\rangle_{L_{w}^{2}\left(\mathrm{C}_{\alpha}\right)}=0$, it holds:

$$
\left\|\varphi_{n} e^{-\frac{1}{h_{n}} f}-c_{n} e^{-\frac{1}{h_{n}}\left(f-\min _{\bar{\Omega}} f\right)}\right\|_{L^{2}\left(\mathrm{C}_{\alpha}\right)}^{2}=\left\|\varphi_{n} e^{-\frac{1}{h_{n}} f}\right\|_{L^{2}\left(\mathrm{C}_{\alpha}\right)}^{2}+c_{n}^{2}\left\|e^{-\frac{1}{h_{n}}\left(f-\min _{\bar{\Omega}} f\right)}\right\|_{L^{2}\left(\mathrm{C}_{\alpha}\right)}^{2} .
$$

Thus, $\lim _{n \rightarrow+\infty}\left\|\varphi_{n} e^{-\frac{1}{h_{n}} f}\right\|_{L^{2}\left(\mathrm{C}_{\alpha}\right)}=0$ which contradicts 42 . This concludes the proof 43).

Step 2: In this step, one proves that in the limit $h \rightarrow 0$ :

$$
\int_{\Omega} u_{1, h} e^{-\frac{2}{h} f}=(\pi h)^{\frac{d}{4}} \sqrt{\sum_{y \in \arg \min _{\bar{\Omega}} f}(\operatorname{det} \operatorname{Hess} f(y))^{-\frac{1}{2}}} e^{-\frac{1}{h} \min _{\bar{\Omega}} f} .
$$


Equation (43) implies that there exists $c>0$ such that for $h$ small enough, $\lambda_{1, h}=O\left(e^{-\frac{c}{h}}\right) \lambda_{2, h}$. Therefore, there exists $\beta_{0}>0$ such that for all $\beta \in\left(0, \beta_{0}\right)$, there exists $h_{0}>0$ such that for all $h \in\left(0, h_{0}\right)$, the $L_{w^{-}}^{2}$ orthogonal projector $\pi_{h}:=\pi_{\left[0, e^{-\frac{2}{h}\left(\min _{\partial \Omega} f-\min \right.} \bar{\Omega} f-\beta\right)}\left(\mathcal{L}_{f, h}^{\text {Dir }}\right)$ has rank 1. From the following spectral estimate

$$
\left\|\left(1-\pi_{h}\right) \frac{\chi}{\|\chi\|_{L_{w}^{2}(\Omega)}}\right\|_{L_{w}^{2}(\Omega)}^{2} \leq C e^{\frac{2}{h}\left(\min _{\partial \Omega} f-\min _{\bar{\Omega}} f-\beta\right)}\left\langle\mathcal{L}_{f, h} \frac{\chi}{\|\chi\|_{L_{w}^{2}(\Omega)}}, \frac{\chi}{\|\chi\|_{L_{w}^{2}(\Omega)}}\right\rangle_{L_{w}^{2}(\Omega)},
$$

and in view of (45), it holds for some $C>0$,

$$
\left\|\left(1-\pi_{h}\right) \frac{\chi}{\|\chi\|_{L_{w}^{2}(\Omega)}}\right\|_{L_{w}^{2}(\Omega)}^{2} \leq C h^{\frac{d}{2}+1} e^{-\frac{2}{h}(\beta-\alpha)} .
$$

Then, choosing $\alpha<\beta$, there exists $c>0$ such that for $h$ small enough

$$
\left\|\left(1-\pi_{h}\right) \frac{\chi}{\|\chi\|_{L_{w}^{2}(\Omega)}}\right\|_{L_{w}^{2}(\Omega)}=O\left(e^{-\frac{c}{h}}\right) .
$$

Thus, there exists $c>0$ such that (using in addition the fact that $u_{1, h}>0$ and $\chi \geq 0$ on $\Omega$ ), $u_{1, h}=\frac{\chi}{\|\chi\|_{L_{w}^{2}(\Omega)}}+O\left(e^{-\frac{c}{h}}\right)$ in $L_{w}^{2}$. This implies that there exists $c>0$ such that for $h$ small enough:

$$
\int_{\Omega} u_{1, h} e^{-\frac{2}{h} f}=\int_{\Omega} \frac{\chi e^{-\frac{2}{h} f}}{\|\chi\|_{L_{w}^{2}(\Omega)}}+O\left(e^{-\frac{c}{h}}\right) \sqrt{\int_{\Omega} e^{-\frac{2}{h} f}}=\int_{\Omega} \frac{\chi}{\|\chi\|_{L_{w}^{2}(\Omega)}} e^{-\frac{2}{h} f}+O\left(e^{-\frac{1}{h}\left(\min _{\bar{\Omega}} f+c\right)}\right) .
$$

The lower bound in 477) is then obtained with a Laplace's method for the terms $\int_{\Omega} \chi e^{-\frac{2}{h} f}$ and $\int_{\Omega} \chi^{2} e^{-\frac{2}{h} f}$.

Step 3: Let us now prove that for all measurable subset $\Sigma$ of $\partial \Omega$, one has:

$$
\limsup _{h \rightarrow 0} h \log \mathbb{P}_{\nu_{h}}\left[X_{\tau_{\Omega^{c}}} \in \Sigma\right] \leq-\left(\min _{\bar{\Sigma}} f-\min _{\partial \Omega} f\right),
$$

where we recall that $\nu_{h}$ is the quasi stationary distribution of the process $(1)$ in $\Omega$ (see (40)). Notice that (48) implies (38) when $X_{0} \sim \nu_{h}$. According to (41) and (47), using the Trace theorem, for any $\delta>0$ there exists $h_{0}>0$ such that for all $h \in\left(0, h_{0}\right)$,

$$
\mathbb{P}_{\nu_{h}}\left[X_{\tau_{\Omega^{c}}} \in \Sigma\right] \leq \frac{h}{2 \lambda_{1, h}}\left\|e^{-\frac{1}{h} f} u_{h}\right\|_{H^{1}(\Omega)} e^{-\frac{1}{h} \min _{\bar{\Sigma}} f} e^{\frac{1}{h} \min _{\bar{\Omega}} f} h^{\frac{d}{2}} .
$$

Let us now prove that there exists $C>0$ such that for $h$ small enough,

$$
\left\|e^{-\frac{1}{h} f} u_{h}\right\|_{H^{1}(\Omega)} \leq C h^{-\frac{3}{2}} \sqrt{\lambda_{1, h}} .
$$

One first has from (39), $\frac{h}{2}\left\|e^{-\frac{1}{h} f} \nabla u_{1, h}\right\|_{L^{2}}^{2}=\lambda_{1, h}$. Moreover, since $u_{1, h}=0$ on $\partial \Omega$, it holds $\nabla_{T}\left(e^{-\frac{1}{h} f} u_{1, h}\right)=0$ on $\partial \Omega$ (where $\nabla_{T}$ is the tangential gradient on $\partial \Omega$ ), the Gaffney inequality (see 47, Corollary 2.1.6]) implies that there exist $C>0$ independent of $h$ such that,

$$
\begin{array}{r}
C\left\|e^{-\frac{1}{h} f} \nabla u_{1, h}\right\|_{H^{1}(\Omega)} \leq\left\|e^{-\frac{1}{h} f} \nabla u_{1, h}\right\|_{L^{2}(\Omega)}+\left\|\operatorname{div}\left(e^{-\frac{1}{h} f} \nabla u_{1, h}\right)\right\|_{L^{2}(\Omega)} \\
+\sum_{i, j=1}^{d}\left\|\partial_{i}\left(e^{-\frac{1}{h} f} \partial_{j} u_{1, h}\right)-\partial_{j}\left(e^{-\frac{1}{h} f} \partial_{i} u_{1, h}\right)\right\|_{L^{2}(\Omega)} .
\end{array}
$$


Therefore, there exist $C^{\prime}>0$ such that for all $h>0$,

$$
\begin{aligned}
\left\|e^{-\frac{1}{h} f} \nabla u_{1, h}\right\|_{H^{1}(\Omega)} \leq & \left(C^{\prime}+\frac{C^{\prime}}{h}\right)\left\|e^{-\frac{1}{h} f} \nabla u_{1, h}\right\|_{L^{2}(\Omega)}+C^{\prime}\left\|e^{-\frac{1}{h} f} \Delta u_{1, h}\right\|_{L^{2}(\Omega)} \\
& \leq \frac{C^{\prime} \sqrt{\lambda_{1, h}}}{h^{3 / 2}}+C^{\prime}\left\|e^{-\frac{1}{h} f} \Delta u_{1, h}\right\|_{L^{2}(\Omega)} .
\end{aligned}
$$

It remains to estimate the term $\left\|e^{-\frac{1}{h} f} \Delta u_{1, h}\right\|_{L^{2}}$. Multiplying $\mathcal{L}_{f, h} u_{1, h}=\lambda_{1, h} u_{1, h}$ by $e^{-\frac{1}{h} f}$ and using (39), one obtains that there exists $C>0$ such that for $h$ small enough,

$$
\left\|e^{-\frac{1}{h} f} \Delta u_{1, h}\right\|_{L^{2}(\Omega)} \leq \frac{2}{h}\left(\|\nabla f\|_{L^{2}(\Omega)}\left\|e^{-\frac{1}{h} f} \nabla u_{1, h}\right\|_{L^{2}(\Omega)}+\lambda_{1, h}\right) \leq \frac{C}{h}\left(\frac{\sqrt{\lambda_{1, h}}}{\sqrt{h}}+\lambda_{1, h}\right) \leq C \frac{\sqrt{\lambda_{1, h}}}{h^{\frac{3}{2}}},
$$

where we used the fact that $\lim _{h \rightarrow 0} \lambda_{1, h}=0$ (which follows from 443 ). This concludes the proof of (50). Equation (49) together with (50) and (43) imply 448).

Step 4: Let $\mathrm{K}$ be compact subset of $\left\{f<\min _{\partial \Omega} f\right\}$. To extend 48 to the case when $x \in \mathrm{K}$, let us prove that for any $F \in \mathcal{C}^{\infty}(\partial \Omega, \mathbb{R})$, there exists $c>0$ such that for $h$ small enough,

$$
\max _{(x, y) \in \mathrm{K} \times \mathrm{K}}\left|\mathbb{E}_{x}\left[F\left(X_{\tau_{\Omega^{c}}}\right)\right]-\mathbb{E}_{y}\left[F\left(X_{\tau_{\Omega^{c}}}\right)\right]\right| \leq e^{-\frac{c}{h}}
$$

Equation (51) is a leveling result on $x \in \Omega \mapsto \mathbb{E}_{x}\left[F\left(X_{\tau_{\Omega^{c}}}\right)\right]$. Let us denote by $v_{h} \in H^{1}(\Omega)$ the unique weak solution to the elliptic boundary value problem

$$
\frac{h}{2} \Delta v_{h}-\nabla f \cdot \nabla v_{h}=0 \text { in } \Omega \text { and } v_{h}=F \text { on } \partial \Omega,
$$

which actually belongs to $\mathcal{C}^{\infty}(\bar{\Omega}, \mathbb{R})$. Moreover, the Dynkin's formula implies that $\forall x \in \bar{\Omega}, v_{h}(x)=$ $\mathbb{E}_{x}\left[F\left(X_{\tau_{\Omega^{c}}}\right)\right]$. Notice that for all $h>0,\left\|v_{h}\right\|_{L^{\infty}(\Omega)} \leq\|F\|_{L^{\infty}(\partial \Omega)}$. With the same arguments as those used to prove (25), for $\alpha>0$ small enough there exist $h_{0}>0$ and $c>0$ such that for all $h \in\left(0, h_{0}\right)$,

$$
\left\|\nabla v_{h}\right\|_{L^{\infty}\left(\mathrm{C}_{\alpha}\right)} \leq e^{-\frac{c}{h}}
$$

Then, since for $\alpha>0$ small enough, the open set $C_{\alpha}$ is path-connected (because it is locally path-connected and connected), this proves (51).

Step 5: Proof of (38).

Step 5.1: Let $\mathrm{K}$ be a compact subset of $\left\{f<\min _{\partial \Omega} f\right\}$. Let us prove that for any $F \in$ $\mathcal{C}^{\infty}(\partial \Omega, \mathbb{R})$, there exists $c>0$ such that for $h$ small enough,

$$
\max _{y \in \mathrm{K}}\left|\mathbb{E}_{y}\left[F\left(X_{\tau_{\Omega^{c}}}\right)\right]-\mathbb{E}_{\nu_{h}}\left[F\left(X_{\tau_{\Omega^{c}}}\right)\right]\right| \leq e^{-\frac{c}{h}}
$$

From (40), it holds for $\alpha>0$ small enough:

$$
\mathbb{E}_{\nu_{h}}\left[F\left(X_{\tau_{\Omega^{c}}}\right)\right]=\frac{\int_{\Omega} v_{h} u_{1, h} e^{-\frac{2}{h} f}}{\int_{\Omega} u_{h} e^{-\frac{2}{h} f}}=\frac{1}{Z_{h}} \int_{\overline{\bar{C}_{\alpha}}} v_{h} u_{1, h} e^{-\frac{2}{h} f}+\frac{1}{Z_{h}} \int_{\Omega \backslash \overline{\bar{C}_{\alpha}}} v_{h} u_{1, h} e^{-\frac{2}{h} f},
$$

where $Z_{h}:=\int_{\Omega} u_{1, h} e^{-\frac{2}{h} f}$. Let us first deal with the second term in (53). From (47), there exists $C>0$ such that for $h$ small enough: $Z_{h}^{-1} \leq C h^{-\frac{d}{2}} e^{\frac{1}{h} \min _{\bar{\Omega}} f}$. For all $\alpha>0$ small enough, one has 
$\left(\bar{\Omega} \backslash \mathrm{C}_{\alpha}\right) \cap \arg \min _{\bar{\Omega}} f=\emptyset$. Therefore, using in addition $(39)$, for all $\alpha>0$ small enough, there exists $c>0$ such that when $h \rightarrow 0$ :

$$
\int_{\Omega \backslash \overline{\mathrm{C}_{\alpha}}} u_{1, h} e^{-\frac{2}{h} f} \leq \sqrt{\int_{\Omega \backslash \overline{\bar{C}_{\alpha}}} e^{-\frac{2}{h} f}} \leq e^{-\frac{1}{h}\left(\min _{\bar{\Omega}} f+c\right)} .
$$

Then, since $\left\|v_{h}\right\|_{L^{\infty}(\Omega)} \leq\|F\|_{L^{\infty}(\partial \Omega)}$, one obtains that

$$
\frac{1}{Z_{h}} \int_{\Omega \backslash \overline{C_{\alpha}}} v_{h} u_{1, h} e^{-\frac{2}{h} f}=O\left(e^{-\frac{c}{h}}\right) .
$$

Let us now deal with the first term in (53). Let $\alpha>0$ be small enough. Then, from (51), there exists $\delta_{\alpha}>0$ such that for all $y \in \overline{\mathrm{C}_{\alpha}}$,

$$
\frac{1}{Z_{h}} \int_{\overline{\bar{C}_{\alpha}}} v_{h} u_{1, h} e^{-\frac{2}{h} f}=\frac{v_{h}(y)}{Z_{h}} \int_{\overline{\bar{C}_{\alpha}}} u_{1, h} e^{-\frac{2}{h} f}+\frac{O\left(e^{-\frac{\delta_{\alpha}}{h}}\right)}{Z_{h}} \int_{\overline{\bar{C}_{\alpha}}} u_{1, h} e^{-\frac{2}{h} f}
$$

in the limit $h \rightarrow 0$ and uniformly with respect to $y \in \overline{\mathrm{C}_{\alpha}}$. Moreover, for all $\alpha>0$ small enough, there exists $c>0$ such that in the limit $h \rightarrow 0$ :

$$
\frac{1}{Z_{h}} \int_{\overline{\mathrm{C}_{\alpha}}} u_{1, h} e^{-\frac{2}{h} f}=1+O\left(e^{-\frac{c}{h}}\right)
$$

This indeed follows from the fact that

$$
\frac{1}{Z_{h}} \int_{\overline{\mathrm{C}_{\alpha}}} u_{1, h} e^{-\frac{2}{h} f}=1-\frac{1}{Z_{h}} \int_{\Omega \backslash \overline{\mathrm{C}_{\alpha}}} u_{1, h} e^{-\frac{2}{h} f},
$$

together with (54). Let us now fix $\alpha>0$ sufficiently small. Then, using (55) and (56), there exist $c>0, \delta_{\alpha}>0$ such that for all $y \in \overline{\mathrm{C}_{\alpha}}$ :

$$
\frac{1}{Z_{h}} \int_{\overline{\bar{C}_{\alpha}}} v_{h} u_{1, h} e^{-\frac{2}{h} f}=v_{h}(y)\left(1+O\left(e^{-\frac{c}{h}}\right)\right)+O\left(e^{-\frac{\delta_{\alpha}}{h}}\right)
$$

in the limit $h \rightarrow 0$ and uniformly with respect to $y \in \overline{\mathrm{C}_{\alpha}}$. Therefore, using (53), (54) and (57), there exists $\alpha_{0}>0$, such that for all $\alpha \in\left(0, \alpha_{0}\right)$, there exists $c>0$ such that for $h$ small enough:

$$
\mathbb{E}_{\nu_{h}}\left[F\left(X_{\tau_{\Omega^{c}}}\right)\right]=\mathbb{E}_{y}\left[F\left(X_{\tau_{\Omega^{c}}}\right)\right]+O\left(e^{-\frac{c}{h}}\right) \text {, uniformly with respect to } y \in \overline{\mathrm{C}_{\alpha}} \text {. }
$$

This proves (52) since any compact $\mathrm{K}$ of $\left\{f<\min _{\partial \Omega} f\right\}$ is included in $\mathrm{C}_{\alpha}$ for some $\alpha>0$.

Step 5.2: End of the proof of (38). To this end, we will use $(48)$ and $(52)$. Let us now consider $\Gamma$ be an open subset of $\partial \Omega$ such that $\min _{\bar{\Gamma}} f>\min _{\partial \Omega} f$. Let us mention that $(52)$ holds for smooth functions $F$ on $\partial \Omega$ and thus we cannot directly consider $F=1_{\Gamma}$. Let us consider an open set $\Sigma \subset \partial \Omega$ such that $\bar{\Gamma} \subset \Sigma$ and $\min _{\bar{\Sigma}} f>\min _{\partial \Omega} f$. Let $F$ be a $\mathcal{C}^{\infty}$ function $F$ on $\partial \Omega$ with values in $[0,1]$ such that $F=1$ on $\Gamma$ and $F=0$ on $\partial \Omega \backslash \Sigma$. For all $y \in \mathbf{K}$ (where $\mathbf{K}$ is a compact subset of $\left\{f<\min _{\partial \Omega} f\right\}$ ), it holds from $(52\}$ :

$$
\mathbb{P}_{y}\left[X_{\tau_{\Omega^{c}}} \in \Gamma\right] \leq \mathbb{E}_{y}\left[F\left(X_{\tau_{\Omega^{c}}}\right)\right]=\mathbb{E}_{\nu_{h}}\left[F\left(X_{\tau_{\Omega^{c}}}\right)\right]+O\left(e^{-\frac{c}{h}}\right) .
$$

Finally, since $\mathbb{E}_{\nu_{h}}\left[F\left(X_{\tau_{\Omega^{c}}}\right)\right] \leq \mathbb{P}_{\nu_{h}}\left[X_{\tau_{\Omega^{c}}} \in \Sigma\right]$, one deduces from [48), that there exists $c>0$ such that for $h$ small enough:

$$
\max _{y \in \mathrm{K}} \mathbb{P}_{y}\left[X_{\tau_{\Omega^{c}}} \in \Gamma\right] \leq e^{-\frac{c}{h}} .
$$

This proves $(38)$ when $\mathrm{K} \subset\left\{f<\min _{\partial \Omega} f\right\}$. The case when $\mathrm{K}$ is a compact subset of $\mathcal{A}_{\Omega}(\{f<$ $\left.\left.\min _{\partial \Omega} f\right\}\right)$ is treated with the same arguments as those used in the fourth step in the proof of Proposition 5 (with the large deviations estimate (33)). This concludes the proof of (38) and the proof of Proposition 7. 


\subsection{Leveling result of $\mathrm{p}_{x_{*}}$ outside $\mathrm{C}\left(x_{*}\right)$}

In this section we prove the following leveling property on $\mathrm{p}_{x_{*}}$ in another sublevel set of $f$ in $\Omega$.

Proposition 8. Let $f: \bar{\Omega} \rightarrow \mathbb{R}$ be a $\mathcal{C}^{\infty}$ Morse function. Let $x_{*, 1}$ and $x_{*, 2}$ be two local minima of $f$ in $\Omega$ such that $\mathrm{C}\left(x_{*, 1}\right) \neq \mathrm{C}\left(x_{*, 2}\right)$ (see (6)). Assume moreover that $\overline{\mathrm{C}\left(x_{*, 2}\right)}$ is a connected component of $\left\{f \leq \lambda\left(x_{*, 2}\right)\right\}$ which intersects $\partial \Omega$. Let $\mathrm{K}$ be a compact set of $\mathcal{A}_{\Omega}\left(\mathrm{C}\left(x_{*, 2}\right)\right)$. Then,

$$
\text { there exist } h_{0}>0 \text { and } c>0 \text { s.t. for all } h \in\left(0, h_{0}\right): \max _{y \in \mathrm{K}} \mathrm{p}_{x_{*, 1}}(y) \leq e^{-\frac{c}{h}} \text {. }
$$

Remark 9. Let us mention that Proposition 8 is not a consequence of [6, Corollary 4.8] for the following two reasons. On the one hand, [6, Corollary 4.8] does not directly apply in our setting because the saddle points connecting $\mathrm{B}_{r_{h}}\left(x_{*, 1}\right)$ and $\Omega^{c}$ belongs to $\Omega^{c}$. On the other hand, even if the inequality in [6, Corollary 4.8] holds, it is possible that the height of the saddle points connecting $x \in \mathrm{C}\left(x_{*, 2}\right)$ and $\mathrm{B}_{r_{h}}\left(x_{*, 1}\right)$ in $\mathbb{R}^{d}$ is equal to the height of the those connecting $x$ and $\Omega^{c}$.

Proof. To prove Proposition 8, the strategy consists in using Proposition 7 with a suitable domain $\Omega_{x_{*}, 2} \subset \Omega$ such that:

(i) $\Omega_{x_{*, 2}}$ is a $\mathcal{C}^{\infty}$ connected open subset of $\Omega$ containing $\mathrm{C}\left(x_{*, 2}\right)$.

(ii) $\partial \Omega_{x_{*, 2}} \cap \partial \Omega$ is a neighborhood of $\partial \mathrm{C}\left(x_{*, 2}\right) \cap \partial \Omega$ in $\partial \Omega$.

(iii) $\operatorname{argmin}_{\partial \Omega_{x_{*, 2}}} f=\partial \mathrm{C}\left(x_{*, 2}\right) \cap \partial \Omega$.

(iv) $\left\{f<\min _{\partial \Omega_{x_{*}, 2}} f\right\} \cap \overline{\Omega_{x_{*, 2}}}=\mathrm{C}\left(x_{*, 2}\right)$ and $\mathrm{C}\left(x_{*, 2}\right)$ contains all the local minima $x$ of $f$ in $\Omega_{x_{*, 2}}$.

(v) $\left\{f \leq \min _{\partial \Omega_{x_{*}, 2}} f\right\} \cap \overline{\Omega_{x_{*, 2}}}=\overline{\mathrm{C}\left(x_{*, 2}\right)}$.

Notice that item (iii) above implies that $\min _{\partial \Omega_{x_{*}, 2}} f=\lambda\left(x_{*, 2}\right)$ (see (6)). Because $\overline{\mathrm{C}\left(x_{*, 2}\right)}$ is a connected component of $\left\{f \leq \lambda\left(x_{*, 2}\right)\right\}$, there exists an open subset $\mathrm{V}\left(x_{*, 2}\right)$ of $\bar{\Omega}$ such that $\overline{\mathrm{C}\left(x_{*, 2}\right)} \subset \mathrm{V}\left(x_{*, 2}\right)$

$$
\left\{f \leq \lambda\left(x_{*, 2}\right)\right\} \cap \mathrm{V}\left(x_{*, 2}\right)=\overline{\mathrm{C}\left(x_{*, 2}\right)} .
$$

Notice that this implies in particular that

$$
\left\{f<\lambda\left(x_{*, 2}\right)\right\} \cap \mathrm{V}\left(x_{*, 2}\right)=\mathrm{C}\left(x_{*, 2}\right) .
$$

Moreover, since $\overline{\mathrm{C}\left(x_{*, 2}\right)} \cap \partial \Omega=\partial \mathrm{C}\left(x_{*, 2}\right) \cap \partial \Omega \neq \emptyset$,

$$
\mathrm{V}\left(x_{*, 2}\right) \cap \partial \Omega \text { is a neighborhood of } \partial \mathrm{C}\left(x_{*, 2}\right) \cap \partial \Omega \text { in } \partial \Omega \text {. }
$$

In addition, because $f$ has a finite number of critical points in $\bar{\Omega}$ and $\partial \mathrm{C}\left(x_{*, 2}\right)$ does not contain local minimum of $f$ in $\Omega$, one can assume that $\overline{\mathrm{V}\left(x_{*, 2}\right)} \backslash \mathrm{C}\left(x_{*, 2}\right)$ does not contain local minimum of $f$ in $\Omega$. In view of all these previous points, there exists a $\mathcal{C}^{\infty}$ connected open subset $\Omega_{x_{*, 2}}$ of $\Omega$ such that:

$$
\mathrm{C}\left(x_{*, 2}\right) \subset \Omega_{x_{*, 2}}, \overline{\Omega_{x_{*}, 2}} \subset \mathrm{V}\left(x_{*, 2}\right),
$$

and such that: the set $\partial \Omega_{x_{*, 2}} \cap \partial \Omega$ is a a neighborhood of $\partial \mathrm{C}\left(x_{*, 2}\right) \cap \partial \Omega$ in $\partial \Omega$ with

$$
\underset{\partial \Omega_{x_{*}, 2} \cap \partial \Omega}{\arg \min } f=\partial \mathrm{C}\left(x_{*, 2}\right) \cap \partial \Omega,
$$


and,

$$
\text { for some } \beta>0, f \geq \lambda\left(x_{*, 2}\right)+\beta \text { on } \overline{\partial \Omega_{x_{*}, 2} \cap \Omega} .
$$

A schematic representation of $\Omega_{x_{*, 2}}$ is given in Figure 3. It is then easy to check that $\Omega_{x_{*, 2}}$ satisfy items (i)-(v) above.

Let us now end the proof of Proposition 8. Since for $h$ small enough, $\mathrm{B}_{r_{h}}\left(x_{*, 1}\right) \cap \overline{\Omega_{x_{*, 2}}}=\emptyset$, using the continuity of the trajectories of the process (1), it holds for all $x \in \mathrm{C}\left(x_{*, 2}\right)$ and $h$ small enough:

$$
\left\{\tau_{\mathrm{B}_{r_{h}}\left(x_{*, 1}\right)}<\tau_{\Omega^{c}}\right\} \subset\left\{X_{\tau_{\Omega_{x_{*}, 2}^{c}}} \in \partial \Omega_{x_{*, 2}} \backslash \partial \Omega\right\} .
$$

The domain $\Omega_{x_{*, 2}}$ and $f: \overline{\Omega_{x_{*}, 2}}: \rightarrow \mathbb{R}$ satisfies all the required assumptions of Proposition 7 . Moreover, since $\inf _{\partial \Omega_{x_{*, 2}} \backslash \partial \Omega} f>\min _{\Omega_{x_{*}, 2}} f=\lambda\left(x_{*, 2}\right)$ (see items (ii) and (iii) above), from Proposition 7, for all compact subset $\mathrm{K}$ of $\mathrm{C}\left(x_{*, 2}\right)=\left\{v \in \overline{\Omega_{x_{*, 2}}}, f(v)<\lambda\left(x_{*, 2}\right)\right\}$ (see item (iv) above), there exists $c>0$ such that for $h$ small enough (see (38)):

$$
\max _{y \in \mathrm{K}} \mathbb{P}_{y}\left[X_{\tau_{\Omega_{x_{*}}^{c}}} \in \partial \Omega_{x_{*, 2}} \backslash \partial \Omega\right] \leq e^{-\frac{c}{h}} .
$$

Recall that by definition (see 10) $\mathbf{p}_{x_{*, 1}}(y)=\mathbb{P}_{x_{*, 1}}\left[\tau_{\mathrm{B}_{r_{h}}\left(x_{*, 1}\right)}<\tau_{\Omega^{c}}\right]$, and thus, there exists $c>0$ such that for $h$ small enough: $\max _{y \in K} \mathrm{p}_{x_{*, 1}}(y) \leq e^{-\frac{c}{h}}$. The proof of Proposition 8 is complete when $\mathrm{K} \subset \mathrm{C}\left(x_{*, 2}\right)$. The proof of Proposition 8 for $\mathrm{K} \subset \mathcal{A}_{\Omega}\left(\mathrm{C}\left(x_{*, 2}\right)\right)$ is obtained using the same procedure as the one used in the fourth step in the proof of Proposition 5 .
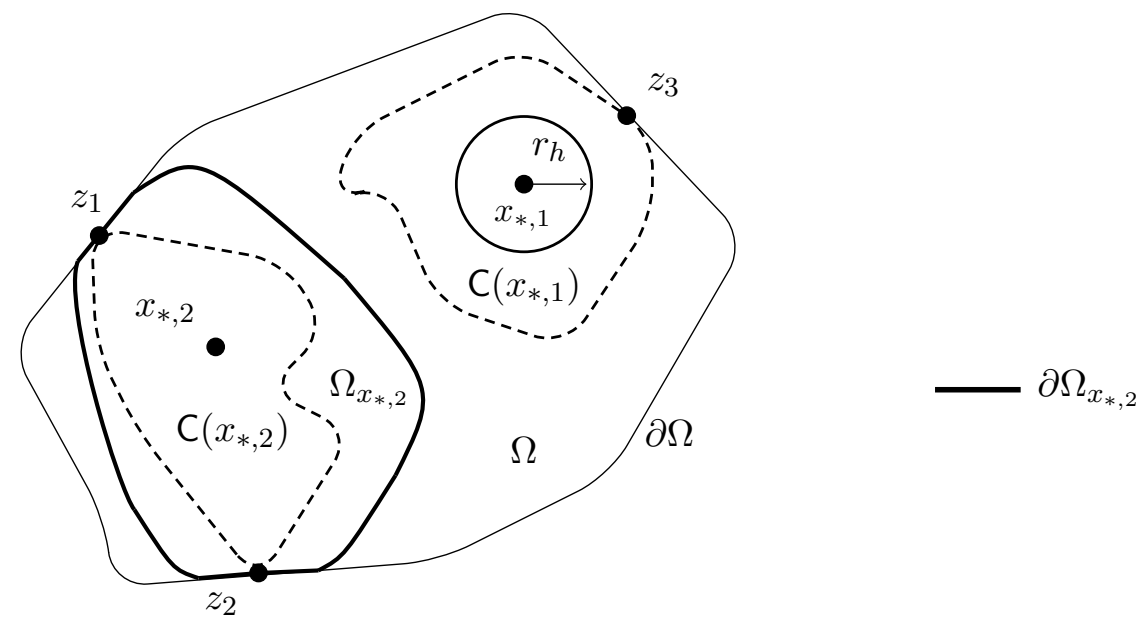

Figure 3: Schematic representation of $\Omega_{x_{*, 2}}$ satisfying items (i)-(v). On the figure, $\partial \mathrm{C}\left(x_{*, 2}\right) \cap$ $\partial \Omega=\left\{z_{1}, z_{2}\right\}$ (see (7)).

A direct consequence of the proof of Proposition 8 is the following generalization of Proposition 7 .

Corollary 10. Let $f: \bar{\Omega} \rightarrow \mathbb{R}$ be a $\mathcal{C}^{\infty}$ Morse function and $x_{*}$ be a local minimum of $f$ in $\Omega$. Assume that $\overline{\mathrm{C}\left(x_{*}\right)}$ (see (7D) is a connected component of $\left\{f \leq \lambda\left(x_{*}\right)\right\}$ which intersects $\partial \Omega$. Let $\mathrm{K}$ be compact subset of $\mathcal{A}_{\Omega}\left(\mathrm{C}\left(x_{*}\right)\right.$ ) (see (5)) and $\Gamma$ be an open subset of $\partial \Omega$. Then, if $\bar{\Gamma} \cap\left(\partial \mathrm{C}\left(x_{*}\right) \cap \partial \Omega\right)=\emptyset$,

there exist $h_{0}>0$ and $c>0$ s.t. for all $h \in\left(0, h_{0}\right): \max _{x \in \mathrm{K}} \mathbb{P}_{x}\left[X_{\tau_{\Omega^{c}}} \in \Gamma\right] \leq e^{-\frac{c}{h}}$. 
Proof. Set $x_{*}=x_{*, 2}$ so that we can use the notation of the proof of Proposition 8 . Since $\bar{\Gamma} \cap\left(\partial \mathrm{C}\left(x_{*, 2}\right) \cap \partial \Omega\right)=\emptyset$, one can assume, up to choosing $\mathrm{V}\left(x_{*, 2}\right)$ smaller, that $\bar{\Gamma} \cap \mathrm{V}\left(x_{*, 2}\right)=\emptyset$ (and therefore, $\bar{\Gamma} \cap \overline{\Omega_{x_{*, 2}}}=\emptyset$ ). Thus, it holds for all $y \in \mathrm{C}\left(x_{*, 2}\right)$, when $X_{0}=y,\left\{X_{\tau_{\Omega^{c}}} \in \Gamma\right\} \subset$ $\left\{X_{\tau_{\Omega_{x_{*}, 2}^{c}}} \in \partial \Omega_{x_{*, 2}} \backslash \partial \Omega\right\}$. Together with (58), this concludes the proof of Corollary 10 when $y \in \mathrm{K} \subset \mathrm{C}\left(x_{*, 2}\right)$. To get it for $\mathrm{K} \subset \mathcal{A}_{\Omega}\left(\mathrm{C}\left(x_{*, 2}\right)\right)$, one uses the procedure of the the fourth step in the proof of Proposition 5

Notice that assumptions of Corollary 10 are satisfied when $\mathbf{( H 1 )}$ holds (see indeed the proof of Lemma 1). Corollary 10 implies that the process (1) exists $\Omega$ almost surely through any neighborhood of $\partial \mathrm{C}\left(x_{*}\right) \cap \partial \Omega$ in the limit $h \rightarrow 0$ when $X_{0}=x \in \mathcal{A}_{\Omega}(\mathrm{C}(x))$.

\subsection{Sharp asymptotic estimate of the capacities}

In this section, one proves an asymptotic equivalent of $\operatorname{cap}_{\mathrm{B}_{r_{h}}\left(x_{*}\right)}\left(\Omega^{c}\right)$ in the limit $h \rightarrow 0$. We start with the following lemma.

Lemma 11. Let $f: \bar{\Omega} \rightarrow \mathbb{R}$ be a $\mathcal{C}^{\infty}$ Morse function and consider a local minimum $x_{*}$ of $f$ in $\Omega$. Let us assume that $\partial \mathrm{C}\left(x_{*}\right) \cap \partial \Omega \neq \emptyset$ (see $\left.(7)\right), \overline{\mathrm{C}\left(x_{*}\right)}$ is a connected component of $\left\{f \leq \lambda\left(x_{*}\right)\right\}$, and that all $z \in \partial \mathrm{C}\left(x_{*}\right) \cap \partial \Omega$ satisfy item 1 or item 2 in $(\mathbf{H 2})$ (depending on $|\nabla f(z)|=0$ or not). Then, there exists a smooth function $\phi_{x_{*}} \in H_{0}^{1}\left(\Omega, e^{-\frac{2}{h} f} d x\right)$ with values in $[0,1]$, supported in a neighborhood of $\mathrm{C}\left(x_{*}\right)$ in $\bar{\Omega}$, which equals 1 in a neighborhood of $x_{*}$ in $\Omega$, such that in the limit $h \rightarrow 0$ :

$$
\begin{aligned}
\frac{h}{2} \int_{\Omega}\left|\nabla \phi_{x_{*}}\right|^{2} e^{-\frac{2}{h} f}= & \sum_{z \in \partial \mathrm{C}\left(x_{*}\right) \cap \partial \Omega,|\nabla f(z)| \neq 0} \frac{(h \pi)^{\frac{d-1}{2}} \partial_{\mathrm{n}} f(z)}{\left(\left.\operatorname{det} \operatorname{Hess} f\right|_{\partial \Omega}(z)\right)^{\frac{1}{2}}} e^{-\frac{2}{h} \lambda\left(x_{*}\right)}(1+O(h)) \\
& +\sum_{z \in \partial \mathrm{C}\left(x_{*}\right) \cap \partial \Omega,|\nabla f(z)|=0} \frac{(h \pi)^{\frac{d}{2}}\left|\mu_{z}\right|}{\pi|\operatorname{det} \operatorname{Hess} f(z)|^{\frac{1}{2}}} e^{-\frac{2}{h} \lambda\left(x_{*}\right)}(1+O(\sqrt{h})) .
\end{aligned}
$$

Proof. The existence of $\phi_{x_{*}} \in H_{0}^{1}\left(\Omega, e^{-\frac{2}{h} f} d x\right)$ with values in $[0,1]$ and supported in a neighborhood of $\overline{\mathrm{C}\left(x_{*}\right)}$ in $\bar{\Omega}$ follows from 30 , Definition 21]. Moreover, because $\overline{\mathrm{C}\left(x_{*}\right)}$ is a connected component of $\left\{f \leq \lambda\left(x_{*}\right)\right\}, \partial \mathrm{C}\left(x_{*}\right) \cap \Omega$ does not contain separating saddle point of $f$ in $\Omega$. Since we assumed that all $z \in \partial \mathrm{C}\left(x_{*}\right) \cap \partial \Omega$ satisfy items 1 or 2 in (H2) (depending on $|\nabla f(z)|=0$ or not), the asymptotic equivalent stated in Lemma 11 on $\int_{\Omega}\left|\nabla \phi_{x_{*}}\right|^{2} e^{-\frac{2}{h} f}$ is then a consequence of [30, Definition 22, Equation (93), and item 1 in Proposition 25] and (3).

The following proposition provides the asymptotic equivalent of $\operatorname{cap}_{\mathrm{B}_{r_{h}}\left(x_{*}\right)}\left(\Omega^{c}\right)$ when $h \rightarrow 0$.

Proposition 12. Let $f: \bar{\Omega} \rightarrow \mathbb{R}$ be a $\mathcal{C}^{\infty}$ Morse function and consider $x_{*}$ a local minimum of $f$ in $\Omega$. Assume that the assumptions on $\mathrm{C}\left(x_{*}\right)$ stated in Lemma 11 are satisfied. Then, it holds, in the limit $h \rightarrow 0$ :

$$
\begin{aligned}
\operatorname{cap}_{\mathrm{B}_{r_{h}}\left(x_{*}\right)}\left(\Omega^{c}\right)= & \sum_{z \in \partial \mathrm{C}\left(x_{*}\right) \cap \partial \Omega,|\nabla f(z)| \neq 0} \frac{(h \pi)^{\frac{d-1}{2}} \partial_{\mathrm{n}} f(z)}{\left(\left.\operatorname{det} \operatorname{Hess} f\right|_{\partial \Omega}(z)\right)^{\frac{1}{2}}} e^{-\frac{2}{h} \lambda\left(x_{*}\right)}(1+O(h)) \\
& +\sum_{z \in \partial \mathrm{C}\left(x_{*}\right) \cap \partial \Omega,|\nabla f(z)|=0} \frac{(h \pi)^{\frac{d}{2}}\left|\mu_{z}\right|}{\pi|\operatorname{det} \operatorname{Hess} f(z)|^{\frac{1}{2}}} e^{-\frac{2}{h} \lambda\left(x_{*}\right)}(1+O(\sqrt{h})) .
\end{aligned}
$$


Proof. From (13), it holds: $\operatorname{cap}_{\mathrm{B}_{r_{h}}\left(x_{*}\right)}\left(\Omega^{c}\right) \leq \frac{h}{2} \int_{\Omega \backslash \mathrm{B}_{r_{h}}\left(x_{*}\right)}\left|\nabla \phi_{x_{*}}\right|^{2} e^{-\frac{2}{h} f}$. Using Lemma 11, this gives the required asymptotic upper bound on $\operatorname{cap}_{\mathrm{B}_{r_{h}}\left(x_{*}\right)}\left(\Omega^{c}\right)$. Let us now obtain a lower bound on $\operatorname{cap}_{\mathrm{B}_{r_{h}}\left(x_{*}\right)}\left(\Omega^{c}\right)$. To this end, we will use 44 . Let us consider two smooth subdomains $\Omega_{x_{*}}^{1}$ and $\Omega_{x_{*}}^{2}$ of $\Omega$, both satisfying items (i)-(v) listed at the beginning of the proof of Proposition 8 , such that $\mathrm{C}\left(x_{*}\right) \subset \Omega_{x_{*}}^{1} \subset \Omega_{x_{*}}^{2}$ and $\overline{\Omega_{x_{*}}^{2}}$ is a neighborhood of $\overline{\Omega_{x_{*}}^{1}}$ in $\bar{\Omega}$. This implies in particular that

$$
\text { there exists } r>0 \text { s.t. for all } x \in \Omega_{x_{*}}^{2} \backslash \overline{\Omega_{x_{*}}^{1}}, f(x) \geq \lambda\left(x_{*}\right)+r .
$$

Let $\chi \in \mathcal{C}^{\infty}(\bar{\Omega},[0,1])$ be such that $\chi=1$ on $\overline{\Omega_{x_{*}}^{1}}$ and $\chi=0$ on $\bar{\Omega} \backslash \overline{\Omega_{x_{*}}^{2}}$. From $(13)$, it holds:

$$
\operatorname{cap}_{\mathrm{B}_{r_{h}}\left(x_{*}\right)}\left(\Omega^{c}\right)=\frac{h}{2} \int_{\Omega \backslash \mathrm{B}_{r_{h}}\left(x_{*}\right)}\left|\nabla \mathrm{p}_{x_{*}}\right|^{2} e^{-\frac{2}{h} f} \geq \frac{h}{2} \int_{\Omega_{x_{*}}^{1} \backslash \mathrm{B}_{r_{h}}\left(x_{*}\right)}\left|\nabla \mathrm{p}_{x_{*}}\right|^{2} e^{-\frac{2}{h} f} .
$$

Let us now obtain an asymptotic lower bound on $\frac{h}{2} \int_{\Omega_{x_{*}}^{1} \backslash \mathrm{B}_{r_{h}}\left(x_{*}\right)}\left|\nabla \mathrm{p}_{x_{*}}\right|^{2} e^{-\frac{2}{h} f}$ in the limit $h \rightarrow 0$. To this end, let us write:

$$
\begin{aligned}
\int_{\Omega_{x_{*}}^{2} \backslash \mathrm{B}_{r_{h}}\left(x_{*}\right)}\left|\nabla\left(\chi \mathrm{p}_{x_{*}}\right)\right|^{2} e^{-\frac{2}{h} f} & =\int_{\Omega_{x_{*} \backslash \mathrm{B}_{r_{h}}\left(x_{*}\right)}}\left(\chi^{2}\left|\nabla \mathrm{p}_{x_{*}}\right|^{2}+2 \mathrm{p}_{x_{*}} \chi \nabla \chi \cdot \nabla \mathrm{p}_{x_{*}}+\mathrm{p}_{x_{*}}^{2}|\nabla \chi|^{2}\right) e^{-\frac{2}{h} f} \\
& =\int_{\Omega_{x_{*}}^{1} \backslash \mathrm{B}_{r_{h}}\left(x_{*}\right)}\left|\nabla \mathrm{p}_{x_{*}}\right|^{2} e^{-\frac{2}{h} f}+R_{*},
\end{aligned}
$$

where

$$
R_{*}=\int_{\Omega_{x_{*}}^{2} \backslash \Omega_{x_{*}}^{1}} \chi^{2}\left|\nabla \mathrm{p}_{x_{*}}\right|^{2} e^{-\frac{2}{h} f}+\int_{\Omega_{x_{*}}^{2} \backslash \Omega_{x_{*}}^{1}}\left(2 \mathrm{p}_{x_{*}} \chi \nabla \chi \cdot \nabla \mathrm{p}_{x_{*}}+\mathrm{p}_{x_{*}}^{2}|\nabla \chi|^{2}\right) e^{-\frac{2}{h} f},
$$

where we used the fact that $\nabla \chi=0$ on $\overline{\Omega_{x_{*}}^{1}}$ and $\chi=1$ on $\overline{\Omega_{x_{*}}^{1}}$. Moreover, from (20), 60, and since $\mathrm{p}_{x_{*}} \leq 1$, there exists $c>0$, such that for $h$ small enough: $R_{*}=O\left(e^{-\frac{2}{h}\left(\lambda\left(x_{*}\right)+c\right)}\right)$. Now since $\chi \mathbf{p}_{x_{*}} \in H_{0}^{1}\left(\Omega_{x_{*}}^{2}, e^{-\frac{2}{h} f} d x\right)$ (because $\mathbf{p}_{x_{*}}=0$ on $\partial \Omega$ and $\chi=0$ on $\partial \Omega_{x_{*}}^{2} \cap \Omega$ ), using the min-max principle (see (2)):

$$
\frac{h}{2} \int_{\Omega_{x_{*}}^{2} \backslash \mathrm{B}_{r_{h}}\left(x_{*}\right)}\left|\nabla\left(\chi \mathbf{p}_{x_{*}}\right)\right|^{2} e^{-\frac{2}{h} f}=\frac{h}{2} \int_{\Omega_{x_{*}}^{2}}\left|\nabla\left(\chi \mathbf{p}_{x_{*}}\right)\right|^{2} e^{-\frac{2}{h} f} \geq \lambda_{1, h} \int_{\Omega_{x_{*}}^{2}}\left|\chi \mathbf{p}_{x_{*}}\right|^{2} e^{-\frac{2}{h} f},
$$

where $\lambda_{1, h}$ denotes here the principal eigenvalue of the Dirichlet realization of $\mathcal{L}_{f, h}$ in $\Omega_{x_{*}}^{2}$. In conclusion, for $h$ small enough:

$$
\operatorname{cap}_{\mathrm{B}_{r_{h}}\left(x_{*}\right)}\left(\Omega^{c}\right) \geq \lambda_{1, h} \int_{\Omega_{x_{*}}^{2}}\left|\chi \mathrm{p}_{x_{*}}\right|^{2} e^{-\frac{2}{h} f}+O\left(e^{-\frac{2}{h}\left(\lambda\left(x_{*}\right)+c\right)}\right) .
$$

Since $\chi=1$ on $\mathrm{C}\left(x_{*}\right)$, from Proposition 5 and since $\arg \min \overline{\mathrm{C}\left(x_{*}\right)} f=\arg \min \frac{}{\Omega_{x_{*}}^{2}} f$ (see item (iv) at the beginning of the proof of Proposition 8), a Laplace's method provides when $h \rightarrow 0$ :

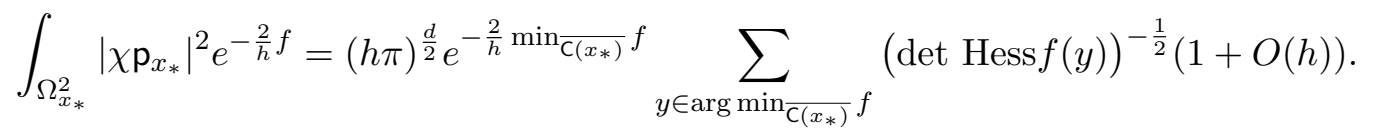

Furthermore, the domain $\Omega_{x_{*}}^{2}$ and $f: \overline{\Omega_{x_{*}}^{2}}: \rightarrow \mathbb{R}$ satisfy all the required assumptions of Proposition 7. Let us recall that for $f: \overline{\Omega_{x_{*}}^{2}}: \rightarrow \mathbb{R}$ it holds $N=1$, and (see items (i)-(iv) at the beginning of the proof of Proposition 8):

$$
\min _{\partial \Omega_{x_{*}}^{2}} f=\lambda\left(x_{*}\right) \text { and } \mathrm{C}_{1}=\left\{v \in \overline{\Omega_{x_{*}}^{2}}, f(v)<\min _{\partial \Omega_{x_{*}}^{2}} f\right\}=\mathrm{C}\left(x_{*}\right) .
$$

Since (H2) holds for $f: \overline{\Omega_{x_{*}}^{2}}: \rightarrow \mathbb{R}$, the asymptotic equivalent of $\lambda_{1, h}$ when $h \rightarrow 0$ given in (44) (with $\Omega=\Omega_{x_{*}}^{2}$ there) holds. Together with (61) and (62), this provides the expected lower bounds on $\operatorname{cap}_{\mathrm{B}_{r_{h}}\left(x_{*}\right)}\left(\Omega^{c}\right)$. The proof of Proposition 12 is complete. 


\section{Proof of Theorems 1 and 2}

In all this section, we assume that the function $f: \bar{\Omega} \rightarrow \mathbb{R}$ satisfies (H1). Then, the results of the previous section can be used with $\mathrm{C}\left(x_{*}\right)=\mathrm{C}_{j}$, where $x_{*}$ is a local minimum of $f$ in $\Omega$ such that $x_{*} \in \mathrm{C}_{j}, j \in\{1, \ldots, \mathrm{N}\}$ (in this case $\lambda\left(x_{*}\right)=\min _{\partial \Omega} f$ ), see Lemma 1 and its proof.

\subsection{Mean value of the commitor functions}

The following proposition gives sharp asymptotic equivalents of $\int_{\Omega} \mathrm{p}_{x_{j}} e^{-\frac{2}{h} f}$ for $j \in\{1, \ldots, \mathrm{N}\}$, where $x_{j}$ is a local minimum of $f$ in $\mathrm{C}_{j}$.

Proposition 13. Let $f: \bar{\Omega} \rightarrow \mathbb{R}$ be such that the assumption (H1) holds. Let $x_{j}$ be a local minimum of $f$ in $\mathrm{C}_{j}$, for some $j \in\{1, \ldots, \mathrm{N}\}$, and $k \in\{1,2\}$. If $j \in\left\{1, \ldots, \mathrm{N}_{0}\right\}$, it holds when $h \rightarrow 0$ :

$$
\int_{\Omega} \mathbf{p}_{x_{j}}^{k} e^{-\frac{2}{h} f}=(h \pi)^{\frac{d}{2}} e^{-\frac{2}{h} \min _{\bar{\Omega}} f} \sum_{y \in \arg \min _{\overline{C_{j}}} f}(\operatorname{det} \operatorname{Hess} f(y))^{-\frac{1}{2}}(1+O(h)),
$$

where we recall that $\mathrm{p}_{x_{j}}$ is defined by (10) with $x_{*}=x_{j}$ there. Finally, if $j \geq \mathrm{N}_{0}+1$, there exists $\varepsilon>0$, such that if $\min _{\overline{\mathrm{C}_{j}}} f-\min _{\bar{\Omega}} f<\varepsilon$, in the limit $h \rightarrow 0$, it holds:

$$
\int_{\Omega} \mathbf{p}_{x_{j}}^{k} e^{-\frac{2}{h} f}=(h \pi)^{\frac{d}{2}} e^{-\frac{2}{h} \min _{\overline{C_{j}}} f} \sum_{y \in \arg \min _{\overline{C_{j}}} f}(\operatorname{det} \operatorname{Hess} f(y))^{-\frac{1}{2}}(1+O(h)) .
$$

Proof. Let $j \in\{1, \ldots, N\}$ and writes:

$$
\int_{\Omega} \mathbf{p}_{x_{j}}^{k} e^{-\frac{2}{h} f}=\int_{\mathbf{C}_{j}} \mathbf{p}_{x_{j}}^{k} e^{-\frac{2}{h} f}+\int_{\Omega \backslash \mathbf{C}_{j}} \mathbf{p}_{x_{j}}^{k} e^{-\frac{2}{h} f} .
$$

To prove Proposition 13, in view of Proposition 5 (applied with $x_{*}=x_{j}$ and $\mathrm{C}\left(x_{*}\right)=\mathrm{C}_{j}$ ), it remains to show that $\int_{\Omega \backslash C_{j}} \mathbf{p}_{x_{j}}^{k} e^{-\frac{2}{h} f}$ is an error term for $h$ small enough. Let us consider a neighborhood $\mathrm{V} \subset \Omega$ of the points belonging to $\arg \min _{\bar{\Omega}} f \backslash \mathrm{C}_{j}$. Let us moreover choose $\mathrm{V}$ such that $\overline{\mathrm{V}} \subset \cup_{\ell \neq j, \ell=1, \ldots, \mathrm{N}_{0}} \mathrm{C}_{\ell}$ (this possible because $\arg \min _{\bar{\Omega}} f \subset \cup_{\ell=1, \ldots, \mathrm{N}_{0}} \mathrm{C}_{\ell}$ according to (8) and the line below). Since there exists $\alpha_{1}>0$ such that $f(x) \geq \min _{\bar{\Omega}} f+\alpha_{1}$ for all $x \in \bar{\Omega} \backslash\left(\mathrm{C}_{j} \cup \mathrm{V}\right)$, it holds, using in addition the fact that $\mathrm{p}_{x_{j}} \leq 1$,

$$
\int_{\Omega \backslash\left(C_{j} \cup \vee\right)} p_{x_{j}}^{k} e^{-\frac{2}{h} f} \leq C e^{-\frac{2}{h}\left(\min _{\bar{\Omega}} f+\alpha_{1}\right)}, \text { for some } C>0 \text { independent of } h .
$$

Since $\overline{\mathrm{V}} \subset \cup_{\ell \neq j, \ell=1, \ldots, \mathrm{N}_{0}} \mathrm{C}_{\ell}$, from Proposition 8 (applied with $x_{1, *}=x_{j}, x_{2, *}=x_{\ell}$, and $\mathrm{K}=\overline{\mathrm{V} \cap \mathrm{C}_{\ell}}$, for $\left.\ell \in\left\{1, \ldots, \mathrm{N}_{0}\right\}, \ell \neq j\right)$, one has:

$$
\int_{\mathbb{V}} \mathbf{p}_{x_{j}}^{k} e^{-\frac{2}{h} f} \leq C e^{-\frac{2}{h}\left(\min _{\bar{\Omega}} f+\alpha_{2}\right)}, \text { for some } \alpha_{2}>0 \text { and } h \text { small enough. }
$$

Recall that $\min _{\overline{C_{j}}} f \geq \min _{\bar{\Omega}} f$ for all $j$, and by definition of $\mathrm{N}_{0}$ (see (8)) and from the labeling of the $\mathrm{C}_{\ell}$ 's, $\min _{\overline{\mathrm{C}_{j}}} f>\min _{\bar{\Omega}} f$ iff $j>\mathrm{N}_{0}$. Therefore, when $j \leq \mathrm{N}_{0}$, one deduces the second statement in Proposition 13. When $j \geq \mathrm{N}_{0}+1$, one sees that if $\min _{\overline{\mathrm{C}_{j}}} f-\min _{\bar{\Omega}} f<\min \left(\alpha_{1}, \alpha_{2}\right)$, ones obtains the last statement in Proposition 13. This concludes the proof of Proposition 13 . 
Let us assume that (H1) and (H2) hold. Let $x_{j}$ be a local minimum of $f$ in $\mathrm{C}_{j}$, for some $j \in$ $\{1, \ldots, \mathrm{N}\}$. Equation (15) and Proposition 12 together with the first statement in Proposition 13 imply that in the limit $h \rightarrow 0$, when $j \in\left\{1, \ldots, \mathrm{N}_{0}\right\}$ :

$$
\mathbb{E}_{x_{j}}\left[\tau_{\Omega^{c}}\right]=\frac{e^{\frac{2}{h}\left(\min _{\partial \Omega} f-\min _{\bar{\Omega}} f\right)}}{\Theta_{j, 1} h^{-\frac{1}{2}}(1+O(h))+\Theta_{j, 2}(1+O(\sqrt{h}))} .
$$

Equation (15), Proposition 12, and the second statement in Proposition 5 together with the fact that $\int_{\Omega} \mathrm{p}_{x_{j}} e^{-\frac{2}{h} f} \geq \int_{\mathrm{C}_{j}} \mathrm{p}_{x_{j}} e^{-\frac{2}{h} f}$, imply that when $j \in\left\{\mathrm{N}_{0}+1, \ldots, \mathrm{N}\right\}$, it holds for $h$ small enough:

$$
\mathbb{E}_{x_{j}}\left[\tau_{\Omega^{c}}\right] \geq \frac{e^{\frac{2}{h}\left(\min _{\partial \Omega} f-\min _{\overline{c_{j}}} f\right)}}{\Theta_{j, 1} h^{-\frac{1}{2}}(1+O(h))+\Theta_{j, 2}(1+O(\sqrt{h}))} .
$$

Moreover, according to the last statement in Proposition (13), Equation (15), and Proposition 12 , there exists $\varepsilon>0$ such that if $\min _{\overline{\mathrm{C}_{j}}} f-\min _{\bar{\Omega}} f<\varepsilon$ then Equation 64 is actually an equality when $h \rightarrow 0$. This proves items 2 and 3 in Theorem 1 when $X_{0}=x_{j}$. To prove Theorem 1 it thus remains to deal with the case when $X_{0}=x \in \mathcal{A}_{\Omega}\left(\mathrm{C}_{j}\right)$. For that purpose, we need an upper bound on $x \in \Omega \mapsto \mathbb{E}_{x}\left[\tau_{\Omega^{c}}\right]$. This is the goal of the next section.

\subsection{Upper bound on the mean exit time}

Proposition 14. Let $f: \bar{\Omega} \rightarrow \mathbb{R}$ such that the hypotheses (H1) and (H2) hold. Let $\mathrm{K}$ be a compact subset of $\Omega$. Then, for any $\delta>0$, there exists $h_{0}>0$ such that for all $h \in\left(0, h_{0}\right)$ :

$$
\max _{x \in \mathrm{K}} \mathbb{E}_{x}\left[\tau_{\Omega^{c}}\right] \leq e^{\frac{2}{h}\left(\min _{\partial \Omega} f-\min _{\bar{\Omega}} f+\delta\right)} .
$$

Notice that Proposition 14 is item 1 in Theorem 1.

Proof. To prove (65), we will use [49, Theorem 1]. To this end, we construct a $\mathcal{C}^{\infty}$ bounded subdomain $\mathrm{D}_{\Omega}$ of $\mathbb{R}^{d}$ containing $\Omega$ such that $|\nabla f| \neq 0$ on $\partial \mathrm{D}_{\Omega}$ : this is indeed required to use [49, Theorem 1].

Step 1: Construction of the domain $D_{\Omega}$ containing $\Omega$. The domain $D_{\Omega}$ is constructed by slightly "extending" $\Omega$ near the critical points of $f$ on $\partial \Omega$. This is made as follows. Since $f: \bar{\Omega} \rightarrow \mathbb{R}$ is a smooth function, it is by definition, the restriction of a smooth function defined on an open neighborhood $\tilde{\Omega}$ of $\bar{\Omega}$ in $\mathbb{R}^{d}$. This extension is still denoted by $f$ in the following. In addition, since $f: \bar{\Omega} \rightarrow \mathbb{R}$ is a Morse function, the critical points of $f$ in $\bar{\Omega}$ are isolated in $\tilde{\Omega}$. Thus, there exists $\varepsilon>0$, for all $z \in \partial \Omega$ such that $|\nabla f(z)|=0$, it holds:

$$
|\nabla f| \neq 0 \text { on } \mathrm{B}_{\varepsilon}(z) \backslash\{z\} .
$$

Up to choosing $\varepsilon>0$ smaller, the following properties are satisfied: the balls $\mathrm{B}_{\varepsilon}(z)$ are two by two disjoint,

$$
\text { for all } \ell \in\{1, \ldots, \mathrm{N}\} \text { and } y \in \partial \mathrm{C}_{\ell} \cap \partial \Omega \text {, if }|\nabla f(y)| \neq 0 \text { then } y \notin \mathrm{B}_{\varepsilon}(z) \text {, }
$$

and there exists a smooth coordinate system $\Psi: y \in \mathrm{B}_{\varepsilon}(z) \mapsto\left(x^{\prime}, x_{d}\right) \in \mathbb{R}^{d}$ such that $\Psi(z)=0$,

$$
\Omega \cap \mathrm{B}_{\varepsilon}(z)=\left\{y \in \mathrm{B}_{\varepsilon}(z), x_{d}(y)<0\right\} \text { and } \partial \Omega \cap \mathrm{B}_{\varepsilon}(z)=\left\{y \in \mathrm{B}_{\varepsilon}(z), x_{d}(y)=0\right\} .
$$


Equation (66) can be indeed satisfied because $f$ has finite number of critical points in $\partial \Omega$ and for all $k \in\{1, \ldots, \mathrm{N}\}, \partial \mathrm{C}_{k} \cap \partial \Omega$ contains a finite number of points when (H1) and (H2) hold. For $\alpha>0$, let us consider a $\mathcal{C}^{\infty}$ function $x^{\prime} \in \mathbb{R}^{d-1} \mapsto \chi\left(x^{\prime}\right) \in[0, \alpha]$, such that:

$$
\chi\left(x^{\prime}\right)=0 \text { for all } x^{\prime} \in \mathbb{R}^{d-1} \backslash \Psi\left(\partial \Omega \cap \mathrm{B}_{\varepsilon / 2}(z)\right) \text { and } \chi\left(x^{\prime}\right)=\alpha \text { for all } x^{\prime} \in \Psi\left(\partial \Omega \cap \mathrm{B}_{\varepsilon / 4}(z)\right) .
$$

Moroever, one chooses $\alpha>0$ small enough such that

$$
\left\{\left(x^{\prime}, \chi\left(x^{\prime}\right)\right), x^{\prime} \in \Psi\left(\Omega \cap \mathrm{B}_{\varepsilon}(z)\right)\right\} \subset \Psi\left(\mathrm{B}_{\varepsilon}(z)\right) .
$$

A schematic representation of $\left\{\left(x^{\prime}, x_{d}\right) \in \Psi\left(\mathrm{B}_{\varepsilon}(z)\right)\right.$, such that $\left.x_{d}<\chi\left(x^{\prime}\right)\right\}$ is given in Figure 4 . Let us define,

$$
\mathrm{O}_{z}:=\Psi^{-1}\left(\left\{\left(x^{\prime}, x_{d}\right) \in \Psi\left(\mathrm{B}_{\varepsilon}(z)\right), \text { such that } x_{d}<\chi\left(x^{\prime}\right)\right\}\right) .
$$

For ease of notation, we omitted to write explicitly the dependency on $z$ for $\Psi, \chi$, and $\alpha$. The bounded subset $\mathrm{D}_{\Omega}$ of $\mathbb{R}^{d}$ is then defined by:

$$
\mathrm{D}_{\Omega}:=\Omega \cup \bigcup_{z \in \partial \Omega,|\nabla f(z)|=0} \mathrm{O}_{z} .
$$

From the definition of $\chi$ it holds:

$$
\partial \mathrm{D}_{\Omega} \backslash \bigcup_{z \in \partial \Omega,|\nabla f(z)|=0} \mathrm{~B}_{\varepsilon / 2}(z)=\partial \Omega \backslash \bigcup_{z \in \partial \Omega,|\nabla f(z)|=0} \mathrm{~B}_{\varepsilon / 2}(z) .
$$

From (67), for all critical point $z$ of $f$ on $\partial \Omega$ (see Figure 4), it holds:

$$
\partial \mathrm{D}_{\Omega} \cap \mathrm{B}_{\varepsilon}(z)=\Psi^{-1}\left(\left\{\left(x^{\prime}, x_{d}\right) \in \Psi\left(\mathrm{B}_{\varepsilon}(z)\right), \text { such that } x_{d}=\chi\left(x^{\prime}\right)\right\}\right) .
$$

The set $\mathrm{D}_{\Omega}$ is thus a smooth open and connected subset of $\mathbb{R}^{d}$. Finally, it holds:

$$
|\nabla f| \neq 0 \text { on } \partial \mathrm{D}_{\Omega}
$$

and all the critical points of $f$ in $\mathrm{D}_{\Omega}$ belong to $\bar{\Omega}$. In the following, we will need other properties on $\mathrm{D}_{\Omega}$ which might require to reduce the parameter $\varepsilon>0$ one again. Let us state these properties. First, up to choosing $\varepsilon>0$ smaller, it holds for all local minima $x \in \partial \Omega$ of $f$ in $\mathbb{R}^{d}$,

$$
\max _{t \in[-\varepsilon, \varepsilon]} f\left(x+t \mathrm{n}_{\Omega}(x)\right)-f(x)<\min _{\partial \Omega} f-\min _{\bar{\Omega}} f,
$$

where we recall that $\mathrm{n}_{\Omega}(x)$ is the unit outward normal to $\Omega$ at $x$. Let us also recall that, for all $j \in\{1, \ldots, \mathrm{N}\}$ and $z \in \partial \mathrm{C}_{j} \cap \partial \Omega$ up to choosing $\varepsilon>0$ smaller, it holds:

$$
\mathrm{B}_{\varepsilon}(z) \cap\left\{f<\min _{\partial \Omega} f\right\}=\mathrm{C}_{j} \cap \mathrm{B}_{\varepsilon}(z)
$$

which follows from the fact that the $\overline{C_{\ell}}$ 's are two by two disjoint (see(H1). Let us now consider, for some $j \in\{1, \ldots, \mathrm{N}\}, z^{*} \in \partial \mathrm{C}_{j} \cap \partial \Omega$ such that $z_{*}$ is a critical point of $f$ in $\mathbb{R}^{d}$ (recall that $f\left(z_{*}\right)=\min _{\partial \Omega} f$ and $z_{*}$ is a saddle point of $f$ in $\mathbb{R}^{d}$ ). By assumption (see point $b$ in (H2) , up to choosing $\varepsilon>0$ smaller,

$$
z_{*} \text { is the unique maximum of } f: t \in[-\varepsilon, \varepsilon] \mapsto f\left(z_{*}+t \mathrm{n}_{\Omega}\left(z_{*}\right)\right) \text {, }
$$


with $z_{*}+t \mathrm{n}_{\Omega}\left(z_{*}\right) \in \Omega$ when $t \in[-\varepsilon, 0)$, and, $z_{*}+t \mathrm{n}_{\Omega}\left(z_{*}\right) \in \mathbb{R}^{d} \backslash \bar{\Omega}$ when $t \in(0, \varepsilon]$. Then, one defines:

$$
\gamma_{*}: t \in[-\varepsilon, \varepsilon] \mapsto z_{*}+t \mathrm{n}_{\Omega}\left(z_{*}\right) .
$$

Hence, since for $t \in[-\varepsilon, 0), z_{*}+t \mathrm{n}_{\Omega}\left(z_{*}\right) \in \Omega$ and $f\left(z_{*}+t \mathrm{n}_{\Omega}\left(z_{*}\right)\right)<f\left(z_{*}\right)=\min _{\partial \Omega} f$, from (71), one deduces that

$$
\text { for all } t \in[-\varepsilon, 0), \gamma_{*}\left(z_{*}+\operatorname{tn}_{\Omega}\left(z_{*}\right)\right) \in \mathrm{C}_{j} \text {, }
$$

Let us define

$$
s_{*}:=\inf \left\{t \in(0, \varepsilon], \gamma_{*}(t) \in \partial \mathrm{D}_{\Omega}\right\} .
$$

Since $z_{*} \notin \partial \mathrm{D}_{\Omega}$ (see $(69)$ and $\chi(0)=\alpha>0$ ), it holds: $s_{*}>0$. A schematic representation of $\gamma_{*}$ is given in Figure 5. The parameter $\varepsilon>0$ defining $\mathrm{D}_{\Omega}$ is now fixed.

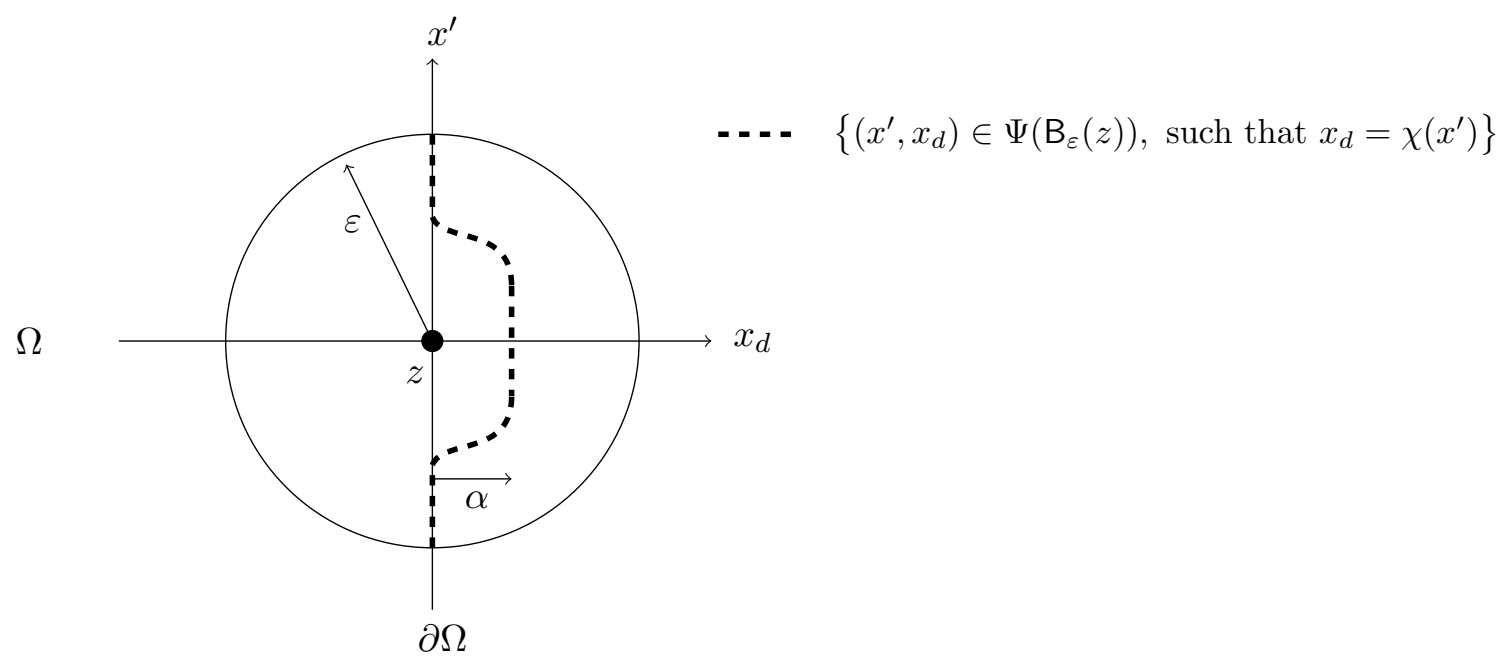

Figure 4: Schematic representation of $\left\{\left(x^{\prime}, x_{d}\right) \in \Psi\left(\mathrm{B}_{\varepsilon}(z)\right)\right.$, such that $\left.x_{d}=\chi\left(x^{\prime}\right)\right\}$ in the $\left(x^{\prime}, x_{d}\right)$ coordinates (see the dashed line).

Step 2: Proof of 65). Let us introduce $\tau_{\mathrm{D}_{\Omega}^{c}}=\inf \left\{t \geq 0, X_{t} \notin \mathrm{D}_{\Omega}\right\}$ the first exit time of the process (1) from $\mathrm{D}_{\Omega}$. Notice that when $X_{0}=x \in \Omega$ it holds $\tau_{\Omega^{c}} \leq \tau_{\mathrm{D}_{\Omega}^{c}}$ (since the trajectories of the process (1) are continuous). Consequently,

$$
\text { for all } x \in \Omega, \mathbb{E}_{x}\left[\tau_{\Omega^{c}}\right] \leq \mathbb{E}_{x}\left[\tau_{\mathrm{D}_{\Omega}^{c}}\right] .
$$

For all local minima $x \in \mathrm{D}_{\Omega}$ of $f$, one defines

$$
\mathrm{H}(x):=\inf \left\{\max _{t \in[0,1]} f(\gamma(t)) \mid \gamma \in C^{0}\left([0,1], \overline{\mathrm{D}_{\Omega}}\right), \gamma(0)=x, \gamma(1) \in \partial \mathrm{D}_{\Omega}\right\}
$$

where $C^{0}\left([0,1], \overline{\mathrm{D}_{\Omega}}\right)$ is the set of continuous paths from $[0,1]$ to $\bar{\Omega}$, and,

$$
\mathrm{H}_{\max }:=\max \left\{\mathrm{H}(x)-f(x), x \in \mathrm{D}_{\Omega} \text { is a local minimum of } f \text { in } \mathrm{D}_{\Omega}\right\} .
$$

From [49, Theorem 1], it holds, for all $\mathrm{K} \subset \Omega$ and for all $\delta>0$, there exists $h_{0}>0$, for all $h \in\left(0, h_{0}\right)$,

$$
\max _{x \in \mathrm{K}} \mathbb{E}_{x}\left[\tau_{\mathrm{D}_{\Omega}^{c}}\right] \leq e^{\frac{2}{h}\left(\mathrm{H}_{\max }+\delta\right)},
$$




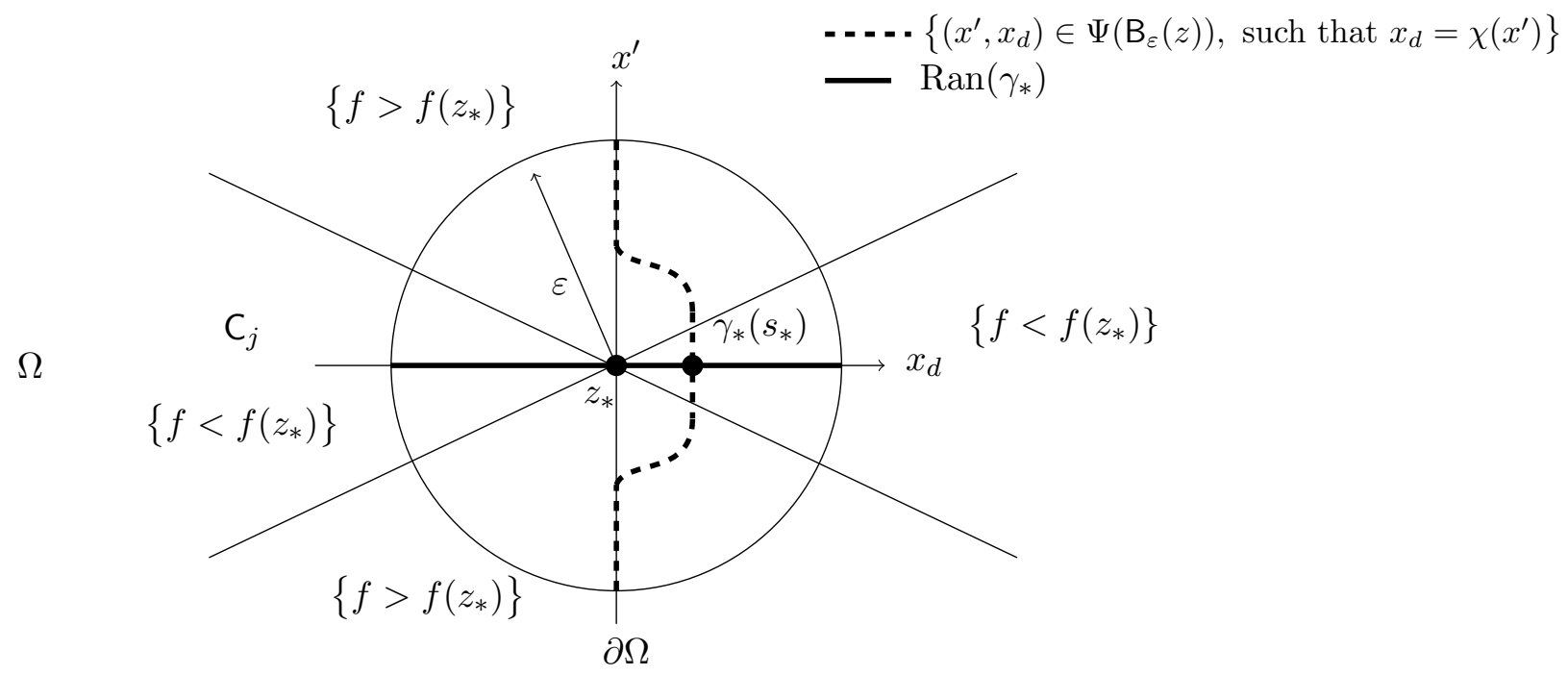

Figure 5: Schematic representation of $\gamma_{*}$ for $z_{*} \in \partial \mathcal{C}_{j} \cap \partial \Omega$ in the $\left(x^{\prime}, x_{d}\right)$ coordinates.

Let us mention that [49, Theorem 1] is not stated uniformly in compact subset $D_{\Omega}$ but this is actually clear from [49, Theorems 3.2 and 4.1]. To prove (65) and in view of (76), let us prove that

$$
\mathrm{H}_{\max }=\min _{\partial \Omega} f-\min _{\bar{\Omega}} f .
$$

Let $x \in \mathrm{D}_{\Omega}$ be a local minimum of $f$ in $\mathrm{D}_{\Omega}$. Then, by construction of $\mathrm{D}_{\Omega}, x \in \bar{\Omega}$. Hence from (H1) $x \in \partial \Omega$ or $x \in \mathrm{C}_{j}$ for some $j \in\{1, \ldots, \mathrm{N}\}$. If $x \in \partial \Omega$, then, from (77), 70, and 69, it holds:

$$
\mathrm{H}(x)-f(x) \leq \max _{t \in[0,1]} f\left(x+\varepsilon \operatorname{tn}_{\Omega}(x)\right)-f(x)<\min _{\partial \Omega} f-\min _{\bar{\Omega}} f .
$$

Let us now consider the case when

$$
x \in \mathrm{C}_{j} \text { for some } j \in\{1, \ldots, \mathrm{N}\} .
$$

Let $\gamma \in C^{0}\left([0,1], \overline{\mathrm{D}_{\Omega}}\right)$ such that $\gamma(0)=x$ and $\gamma(1) \in \partial \mathrm{D}_{\Omega}$. Because $\gamma$ is continuous and $\mathrm{C}_{j} \subset \mathrm{D}_{\Omega}$, there exists $t_{*} \in[0,1]$ such that $\gamma\left(t_{*}\right) \in \partial \mathrm{C}_{j}$. Hence, one has:

$$
\max _{t \in[0,1]} f(\gamma(t)) \geq f\left(\gamma\left(t_{*}\right)\right)=\min _{\partial \Omega} f .
$$

Let us now prove that there exists $T_{1}<T_{2}$, and $\gamma \in C^{0}\left(\left[T_{1}, T_{2}\right], \overline{\mathrm{D}_{\Omega}}\right)$ such that $\gamma\left(T_{1}\right)=x$, $\gamma\left(T_{2}\right) \in \partial \mathrm{D}_{\Omega}$, and

$$
\max _{t \in\left[T_{1}, T_{2}\right]} f(\gamma(t))=\min _{\partial \Omega} f .
$$

Case 1: $\partial \mathrm{C}_{j} \cap \partial \Omega$ contains a critical point $z_{*}$ of $f$.

Let $z_{*} \in \partial \mathrm{C}_{j} \cap \partial \Omega$ be a critical point of $f$. Since $\gamma_{*}(-\varepsilon) \in \mathrm{C}_{j}$ (see (73) and (74)) and $\mathrm{C}_{j}$ is path-connected (because it is open, connected and locally path-connected), there exists a curve $\gamma_{0}:[-2 \varepsilon,-\varepsilon] \rightarrow C_{j}$ such that $\gamma_{0}(-2 \varepsilon)=x$ and $\gamma_{0}(-\varepsilon)=\gamma_{*}(-\varepsilon)$. Let us finally define the continuous curve $\gamma:\left[-2 \varepsilon, s_{*}\right] \rightarrow \overline{\mathrm{D}_{\Omega}}$ by:

for all $t \in[-2 \varepsilon,-\varepsilon], \gamma(t)=\gamma_{0}(t)$, and, for all $t \in\left(-\varepsilon, s_{*}\right], \gamma(t)=\gamma_{*}(t)$, 
where $s_{*}>0$ is defined by (75). It then follows from $(72)$ together with the fact that $\operatorname{Ran}\left(\gamma_{0}\right) \subset$ $\mathrm{C}_{j} \subset\left\{f<\min _{\partial \Omega} f\right\}$ that $\max _{t \in\left[-2 \varepsilon, s_{*}\right]} f(\gamma(t))=f\left(z_{*}\right)=\min _{\partial \Omega} f$. This proves (81) in this case.

Case 2: $\partial \mathrm{C}_{j} \cap \partial \Omega$ does not contain critical point of $f$.

Because, by assumption (see (H1),$\partial \mathrm{C}_{j} \cap \partial \Omega$ is non empty, there exists $z_{*} \in \partial \mathrm{C}_{j} \cap \partial \Omega$. Notice that from (66) and (68), $z_{*} \in \partial \mathrm{D}_{\Omega}$. In addition, since $\partial_{\mathrm{n}} f\left(z_{*}\right)>0$, for $t>0$ small enough, $\varphi_{t}\left(z_{*}\right) \in \Omega$ (see (4)). Furthermore, because there is no critical point of $f$ in a neighborhood of $z_{*}$ in $\mathbb{R}^{d}$, it holds for all $t>0$ sufficiently small,

$$
f\left(\varphi_{t}\left(z_{*}\right)\right)-f\left(z_{*}\right)=\int_{0}^{t} \frac{d}{d s} f\left(\varphi_{s}\left(z_{*}\right)\right) d s=-\int_{0}^{t}|\nabla f|^{2}\left(\varphi_{s}\left(z_{*}\right)\right) d s<0 .
$$

Thus, from (71), for all $t>0$ sufficiently small, $\varphi_{t}\left(z_{*}\right) \in \mathrm{C}_{j}$ (and since $\mathrm{C}_{j}$ is stable for the dynamics (4), $\varphi_{t}\left(z_{*}\right) \in \mathrm{C}_{j}$ for all $\left.t>0\right)$. Let us consider $t_{*}>0$ and a curve $\gamma_{0}:\left[-2 t_{*},-t_{*}\right] \rightarrow \mathrm{C}_{j}$ such that $\gamma_{0}\left(-2 t_{*}\right)=x$ and $\gamma_{0}\left(-t_{*}\right)=\varphi_{t_{*}}\left(z_{*}\right)$. Then, one defines $\gamma:\left[-2 t_{*}, 0\right] \rightarrow \overline{\mathrm{D}_{\Omega}}$ by:

for all $t \in\left[-2 t_{*},-t_{*}\right], \gamma(t)=\gamma_{0}(t)$, and, for all $t \in\left(-t_{*}, 0\right], \gamma(t)=\varphi_{-t}\left(z_{*}\right)$.

By construction, it holds, $\max _{t \in\left[-2 t_{*}, 0\right]} f(\gamma(t))=f\left(z_{*}\right)=\min _{\partial \Omega} f$. This concludes the proof of 81 ).

From (77), (80), and (81), one deduces that for all local minimum $x$ of $f$ in $\Omega, \mathrm{H}(x)=\min _{\partial \Omega} f$. Together with $(79)$ and by definition of $\mathrm{H}_{\max }$, it holds

$$
\mathrm{H}_{\max }=\min _{\partial \Omega} f-\min _{\bar{\Omega}} f
$$

Then, (76) and (78) conclude the proof of Proposition 14 .

\subsection{Proof of Theorem 2 and end of the proof of Theorem 1}

End of the proof of Theorems 1 and Q Let $f: \bar{\Omega} \rightarrow \mathbb{R}$ which satisfies the assumptions (H1) and (H2). Let $j \in\{1, \ldots, \mathrm{N}\}, \mathrm{K}$ be compact subset of $\mathcal{A}_{\Omega}\left(\mathrm{C}_{j}\right)$ and $x \in \mathrm{K}$. Let $x_{j}$ be a local minimum of $f$ in $\mathrm{C}_{j}$. Let us write

$$
\mathbb{E}_{x}\left[\tau_{\Omega^{c}}\right]=\mathbb{E}_{x}\left[\tau_{\Omega^{c}} \mathbf{1}_{\tau_{\mathrm{B}_{r_{h}}\left(x_{j}\right)}<\tau_{\Omega^{c}}}\right]+\mathbb{E}_{x}\left[\tau_{\Omega^{c}} \mathbf{1}_{\tau_{\mathrm{B}_{r_{h}}\left(x_{j}\right)} \geq \tau_{\Omega^{c}}}\right]
$$

By the strong Markov Property,

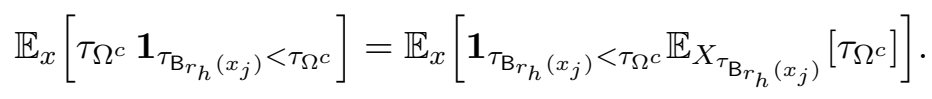

Using Lemma 3 and Proposition 5 , there exists $c>0$ such that for all $h$ small enough and $x \in \mathrm{K}$, it holds:

$$
\mathbb{E}_{x}\left[\tau_{\Omega^{c}} \mathbf{1}_{\tau_{\mathrm{B}_{r_{h}}\left(x_{j}\right)}<\tau_{\Omega^{c}}}\right]=\left(1+O\left(e^{-\frac{c}{h}}\right)\right) \times \mathbb{E}_{x_{j}}\left[\tau_{\Omega^{c}}\right] \text {, uniformly in } x \in \mathrm{K} .
$$

Let us now deal with the last term in $(82)$. Let $\Omega_{\mathrm{K}}$ be as introduced in Proposition 6 (applied with $\mathrm{C}\left(x_{*}\right)=\mathrm{C}_{j}$ ). Since $\Omega_{\mathrm{K}} \subset \Omega, \mathrm{B}_{r_{h}}\left(x_{j}\right) \subset \Omega_{\mathrm{K}}$ (for $h$ small enough) and by continuity of the trajectories of the process (1), it holds for $h$ small enough and for all $x \in \mathrm{K}$,

$$
\left\{\tau_{\mathrm{B}_{r_{h}}\left(x_{j}\right)} \geq \tau_{\Omega^{c}}\right\} \subset\left\{\tau_{\mathrm{B}_{r_{h}}\left(x_{j}\right)} \geq \tau_{\Omega_{\mathrm{K}}^{c}}\right\}, \text { when } X_{0}=x .
$$


Consequently, and using the strong Markov property, for all $x \in \mathrm{K}$, one has

$$
\mathbb{E}_{x}\left[\tau_{\Omega^{c}} \mathbf{1}_{\tau_{\mathrm{B}_{r_{h}}\left(x_{j}\right)} \geq \tau_{\Omega^{c}}}\right] \leq \mathbb{E}_{x}\left[\tau_{\Omega^{c}} \mathbf{1}_{\tau_{\mathrm{B}_{r_{h}}\left(x_{j}\right)} \geq \tau_{\Omega_{\mathrm{K}}^{c}}}\right]=\mathbb{E}_{x}\left[\mathbf{1}_{\tau_{\mathrm{B}_{r_{h}}\left(x_{j}\right)} \geq \tau_{\Omega_{\mathrm{K}}^{c}}} \mathbb{E}_{X_{\tau_{\Omega_{\mathrm{K}}^{c}}}}\left[\tau_{\Omega^{c}}\right]\right] .
$$

Then, using Proposition 6 (with $x_{*}=x_{j}$ and thus, $\mathrm{C}\left(x_{*}\right)=\mathrm{C}_{j}$ ) and Proposition 14 (with the compact set $\overline{\Omega_{\mathrm{K}}}$ there), there exists $c>0$ such that for all $h$ small enough and all $x \in \mathrm{K}$, it holds:

$$
\mathbb{E}_{x}\left[\tau_{\Omega^{c}} \mathbf{1}_{\tau_{\mathrm{B}_{r_{h}}\left(x_{j}\right)} \geq \tau_{\Omega^{c}}}\right] \leq \max _{y \in \mathrm{K}} \mathbb{P}_{y}\left[\tau_{\mathrm{B}_{r_{h}}\left(x_{j}\right)} \geq \tau_{\Omega_{\mathrm{K}}^{c}}\right] \times \max _{y \in \bar{\Omega}_{\mathrm{K}}} \mathbb{E}_{y}\left[\tau_{\Omega^{c}}\right] \leq e^{\frac{2}{h}\left(\min _{\partial \Omega} f-\min _{\bar{\Omega}} f\right)} e^{-\frac{c}{h}},
$$

uniformly in $x \in \mathrm{K}$. Thus, (83), (84), (63), and (82) imply that for all $h$ small enough and $x \in \mathrm{K}$, it holds when $j \in\left\{1, \ldots, \mathrm{N}_{0}\right\}$ :

$$
\mathbb{E}_{x}\left[\tau_{\Omega^{c}}\right]=\mathbb{E}_{x_{j}}\left[\tau_{\Omega^{c}}\right]\left(1+O\left(e^{-\frac{c}{h}}\right)\right)=\frac{e^{\frac{2}{h}\left(\min _{\partial \Omega} f-\min _{\bar{\Omega}} f\right)}}{\Theta_{j, 1} h^{-\frac{1}{2}}(1+O(h))+\Theta_{j, 2}(1+O(\sqrt{h}))},
$$

uniformly in $x \in \mathrm{K}$. When $j \in\left\{\mathrm{N}_{0}+1, \ldots, \mathrm{N}\right\}$, from (82), (83), and (64), it holds when $h \rightarrow 0$ :

$$
\mathbb{E}_{x}\left[\tau_{\Omega^{c}}\right] \geq \frac{e^{\frac{2}{h}\left(\min _{\partial \Omega} f-\min _{\overline{c_{j}}} f\right)}}{\Theta_{j, 1} h^{-\frac{1}{2}}(1+O(h))+\Theta_{j, 2}(1+O(\sqrt{h}))}, \text { uniformly in } x \in \mathrm{K} .
$$

Finally, from (82), 83), 84), and the lines just after (64), there exists $\varepsilon>0$, such that if $\min _{\overline{C_{j}}} f-\min _{\bar{\Omega}} f<\varepsilon$, the previous inequality is an equality which holds uniformly in $x \in \mathrm{K}$. This concludes the proof of Theorem 2, and the proofs of items 2 and 3 in Theorem 1 1 . The proof of Theorem 1 is thus complete.

\section{Appendix}

Appendix 1. In this section, we show the optimality of the errors term $O(\sqrt{h})$ and $O(h)$ in items 2 and 3 in Theorem 1. For that purpose, let us consider a function $f \in \mathcal{C}^{\infty}\left(\left[z_{1}, z_{2}\right], \mathbb{R}\right)$ such that $\left\{z \in\left[z_{1}, z_{2}\right], f^{\prime}(z)=0\right\}=\left\{z_{1}, x_{1}, z\right\}, f^{\prime \prime}\left(z_{1}\right)<0, f^{\prime \prime}(z)<0, f^{\prime \prime}\left(x_{1}\right)>0, f^{\prime}\left(z_{2}\right)<0$, $z_{1}<x_{1}<z<z_{2}$, and $f\left(x_{1}\right)<f\left(z_{1}\right)<f\left(z_{2}\right)<f(z)$ (see Figure 6). Assumption (H1) is thus satisfied with $\mathbf{N}=1$. The following formula holds for $x \in\left[z_{1}, z_{2}\right]$ :

$$
\mathbb{E}_{x}\left[\tau_{\left(z_{1}, z_{2}\right)^{c}}\right]=\frac{2}{h} \int_{z_{1}}^{z_{2}} \int_{z_{1}}^{y} e^{\frac{2}{h}(f(y)-f(t))} d t d y \frac{\int_{z_{1}}^{x} e^{\frac{2}{h} f}}{\int_{z_{1}}^{z_{2}} e^{\frac{2}{h} f}}-\frac{2}{h} \int_{z_{1}}^{x} \int_{z_{1}}^{y} e^{\frac{2}{h}(f(y)-f(t))} d t d y
$$

This formula is a consequence of the Dynkin's formula: the function $x \in\left[z_{1}, z_{2}\right] \mapsto \mathbb{E}_{x}\left[\tau_{\left(z_{1}, z_{2}\right)^{c}}\right]$ is the $\mathcal{C}^{\infty}\left(\left[z_{1}, z_{2}\right], \mathbb{R}\right)$ solution to

$$
\frac{h}{2} e^{\frac{2}{h} f} \frac{d}{d x}\left(e^{-\frac{2}{h} f} \frac{d}{d x} v\right)=-1 \text { in } \Omega, \text { with } v\left(z_{1}\right)=0 \text { and } v\left(z_{2}\right)=0 .
$$

Notice that $\int_{z_{1}}^{x_{1}} \int_{z_{1}}^{y} e^{\frac{2}{h}(f(y)-f(t))} d t d y \leq\left(z_{1}-x_{1}\right)^{2} / 2$ because for $t$ and $y$ such that $z_{1} \leq t \leq y \leq x_{1}$, it holds $f(y)-f(t) \leq 0$. Using a Laplace's method, since the maximum value of $t<y \in\left[z_{1}, z_{2}\right] \mapsto$ $f(y)-f(t)$ attains uniquely is maximum at the point $\left(x_{1}, z\right) \in\left\{t<y,(t, y) \in\left(z_{1}, z_{2}\right)^{2}\right\}$, it holds when $h \rightarrow 0$ :

$$
\int_{z_{1}}^{z_{2}} \int_{z_{1}}^{y} e^{\frac{2}{h}(f(y)-f(t))} d t d y=\frac{\pi h}{\sqrt{f^{\prime \prime}\left(x_{1}\right)\left|f^{\prime \prime}(z)\right|}} e^{\frac{2}{h}\left(f(z)-f\left(x_{1}\right)\right)}(1+O(h)) .
$$


Moreover, since the maximum value of $y \in\left[z_{1}, z_{2}\right] \mapsto f(y)$ is uniquely attained when $y=z \in$ $\left(z_{1}, z_{2}\right)$, one has using again a Laplace's method, in the limit $h \rightarrow 0$ :

$$
\int_{z_{1}}^{z_{2}} e^{\frac{2}{h} f}=\frac{\sqrt{\pi h}}{\sqrt{\left|f^{\prime \prime}(z)\right|}} e^{\frac{2}{h} f(z)}(1+O(h)) .
$$

Finally, since the maximum value of $y \in\left[z_{1}, x_{1}\right] \mapsto f(y)$ is uniquely attained when $y=z_{1} \in$ $\partial\left(z_{1}, x_{1}\right), f^{\prime}\left(z_{1}\right)=0$, and $f^{\prime \prime}\left(z_{1}\right)<0$, using Watson's lemma, it holds when $h \rightarrow 0$ :

$$
\int_{z_{1}}^{x_{1}} e^{\frac{2}{h} f}=\frac{1}{2} \frac{\sqrt{\pi h}}{\sqrt{\left|f^{\prime \prime}\left(z_{1}\right)\right|}} e^{\frac{2}{h} f\left(z_{1}\right)}(1+c \sqrt{h}+O(h)),
$$

where $c \in \mathbb{R}$ can be expressed in terms of the gamma function and the derivatives of $f$ at $z_{1}$. In conclusion, it holds in the limit $h \rightarrow 0$ :

$$
\mathbb{E}_{x}\left[\tau_{\left(z_{1}, z_{2}\right)^{c}}\right]=\frac{\pi e^{\frac{2}{h}\left(f\left(z_{1}\right)-f\left(x_{1}\right)\right)}}{\sqrt{f^{\prime \prime}\left(x_{1}\right)\left|f^{\prime \prime}\left(z_{1}\right)\right|}}(1+c \sqrt{h}+O(h))=\frac{(1+c \sqrt{h}+O(h))}{\Theta_{2,1}} e^{\frac{2}{h}\left(f\left(z_{1}\right)-f\left(x_{1}\right)\right)},
$$

which that shows that the error term $O(\sqrt{h})$ in Theorem 1 is optimal. If we now assume that $f^{\prime}\left(z_{1}\right)<0$, we obtain that, using a Laplace's method, when $h \rightarrow 0$ :

$$
\int_{z_{1}}^{x_{1}} e^{\frac{2}{h} f}=\frac{h}{2\left|f^{\prime}\left(z_{1}\right)\right|} e^{\frac{2}{h} f\left(z_{1}\right)}(1+O(h)), \text { for some } c \in \mathbb{R} .
$$

and thus, in the limit $h \rightarrow 0$ :

$$
\mathbb{E}_{x}\left[\tau_{\left(z_{1}, z_{2}\right)^{c}}\right]=\frac{\sqrt{h \pi} e^{\frac{2}{h}\left(f\left(z_{1}\right)-f\left(x_{1}\right)\right)}}{\left|f^{\prime}\left(z_{1}\right)\right| \sqrt{f^{\prime \prime}\left(x_{1}\right)}}(1+O(h))=\sqrt{h} \frac{(1+O(h))}{\Theta_{1,1}} e^{\frac{2}{h}\left(f\left(z_{1}\right)-f\left(x_{1}\right)\right)},
$$

which shows that the error term $O(h)$ in Theorem 1 is optimal.

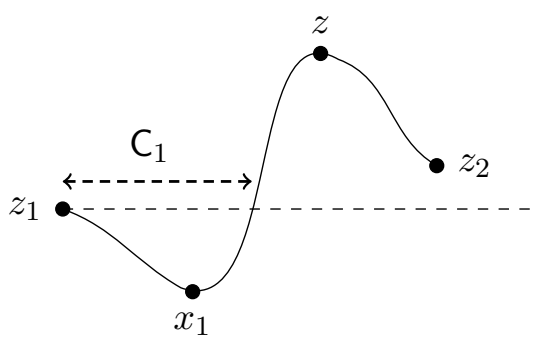

Figure 6: $A$ one dimensional case $(\mathbf{H 1})$ is satisfied with $\mathrm{N}=1\left(\partial \mathrm{C}_{1} \cap \partial \Omega=\left\{z_{1}\right\}\right)$.

Appendix 2. In this section, we discuss the strong tunnelling effect which occurs when a well $C_{i}$ of $f$ in $\Omega$ is much deeper than the other wells $C_{j}, j \neq i$. To this end, we consider a function $f \in \mathcal{C}^{\infty}\left(\left[z_{1}, z_{2}\right], \mathbb{R}\right)$ such that $\left\{z \in\left[z_{1}, z_{2}\right], f^{\prime}(z)=0\right\}=\left\{x_{2}, z, x_{1}\right\}, z_{1}<x_{1}<z<x_{2}<z_{2}$, $f^{\prime \prime}(z)<0, f^{\prime \prime}\left(x_{1}\right)>0, f^{\prime \prime}\left(x_{2}\right)>0, f^{\prime}\left(z_{2}\right)<0, f^{\prime}\left(z_{1}\right)>0$, and $f\left(x_{1}\right)<f\left(x_{2}\right)<f\left(z_{1}\right)=$ $f\left(z_{2}\right)<f(z)$. Such a function $f$ is represented in Figure 7. It thus holds $f\left(x_{2}\right)=\min _{\overline{C_{2}}} f>$ $\min _{\bar{\Omega}} f=f\left(x_{1}\right)$, and $\left\{f<\min _{\partial \Omega} f\right\}$ has two connected components: $\mathrm{C}_{1}$ containing $x_{1}$ and $\mathrm{C}_{2}$ containing $x_{2}$. Moroever, (H1) holds with $\mathrm{N}_{0}=1<\mathrm{N}=2$. When $f(z)-f\left(x_{2}\right)>f\left(z_{1}\right)-f\left(x_{1}\right)$, one has, using Laplace's method in 85 :

$$
\lim _{h \rightarrow 0} h \ln \mathbb{E}_{x_{2}}\left[\tau_{\left(z_{1}, z_{2}\right)^{c}}\right]=2\left(f\left(z_{2}\right)-f\left(x_{2}\right)\right),
$$


whereas, when $f(z)-f\left(x_{2}\right)<f\left(z_{1}\right)-f\left(x_{1}\right)$, it holds:

$$
\lim _{h \rightarrow 0} h \ln \mathbb{E}_{x_{2}}\left[\tau_{\left(z_{1}, z_{2}\right)^{c}}\right]=2\left(f\left(z_{1}\right)-f\left(x_{1}\right)-\left(f(z)-f\left(z_{2}\right)\right)\right) .
$$

We thus observe that when $f(z)-f\left(x_{2}\right)<f\left(z_{1}\right)-f\left(x_{1}\right)$, even if $\overline{C_{1}} \cap \overline{C_{2}}=\emptyset$, a strong tunnelling effect between the wells $\mathrm{C}_{1}$ and $\mathrm{C}_{2}$ occurs. Let us mention that the conclusion remains unchanged if $f^{\prime}\left(z_{1}\right)=0$ or $f^{\prime}\left(z_{2}\right)=0$.

Remark 15. In the example depicted in Figure 1, it has been proved in [30, Theorem 2], that $\lim _{h \rightarrow 0} h \ln \lambda_{1, h}=-2\left(f\left(z_{1}\right)-f\left(x_{1}\right)\right)$ and $\lim _{h \rightarrow 0} h \ln \lambda_{2, h}=-2\left(f\left(z_{2}\right)-f\left(x_{2}\right)\right)$. Thus, when $f(z)-f\left(x_{2}\right)<f\left(z_{1}\right)-f\left(x_{1}\right)$, for all $i \in\{1,2\}, \lim _{h \rightarrow 0} h \ln \mathbb{E}_{x_{2}}\left[\tau_{\left(z_{1}, z_{2}\right)}\right] \neq \lim _{h \rightarrow 0} h \ln \lambda_{i}^{-1}$.

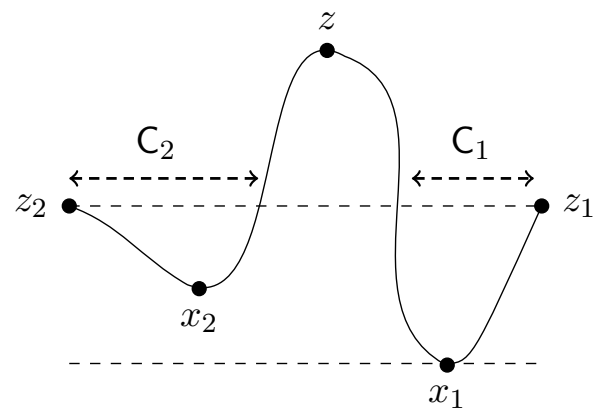

Figure 7: $A$ one dimensional case when $(\mathbf{H 1})$ holds with $\mathrm{N}_{0}=1$ and $\mathrm{N}=2$.

Acknowledgement. The author is grateful to Dorian Le Peutrec for fruitful discussions, especially for his help in the proof of $(43)$ above.

\section{References}

[1] N. Berglund. Kramers' law: validity, derivations and generalisations. Markov Process. Related Fields, 19(3):459-490, 2013.

[2] N. Berglund and S. Dutercq. The Eyring-Kramers law for Markovian jump processes with symmetries. J. Theoret. Probab., 29(4):1240-1279, 2016.

[3] N. Berglund and B. Gentz. The Eyring-Kramers law for potentials with nonquadratic saddles. Markov Process. Related Fields, 16(3):549-598, 2010.

[4] D. Borisov and O. Sultanoc. Asymptotic analysis of exit time for dynamical systems with a single well potential. Journal of Differential Equations, 269(8):78-116, 2019.

[5] A. Bovier and F. Den Hollander. Metastability: a potential-theoretic approach. Springer, 2016.

[6] A. Bovier, M. Eckhoff, V. Gayrard, and M. Klein. Metastability in reversible diffusion processes. I. Sharp asymptotics for capacities and exit times. J. Eur. Math. Soc., 6(4):399-424, 2004.

[7] A. Bovier, V. Gayrard, and M. Klein. Metastability in reversible diffusion processes. II. Precise asymptotics for small eigenvalues. J. Eur. Math. Soc., 7(1):69-99, 2005.

[8] M.V. Day. Exponential leveling for stochastically perturbed dynamical systems. SIAM Journal on Mathematical Analysis, 13(4):532-540, 1982.

[9] M.V. Day. On the exponential exit law in the small parameter exit problem. Stochastics, 8(4):297-323, 1983.

[10] M.V. Day. Mathematical approaches to the problem of noise-induced exit. In Stochastic Analysis, Control, Optimization and Applications, pages 269-287. Springer, 1999. 
[11] A. Devinatz and A. Friedman. Asymptotic behavior of the principal eigenfunction for a singularly perturbed dirichlet problem. Indiana Univ. Math. J., 27:143-157, 1978.

[12] A. Devinatz and A. Friedman. Asymptotic behavior of the principal eigenfunction for a singularly perturbed dirichlet problem. Indiana University Mathematics Journal, 27:143-157, 1978.

[13] G. Di Gesù, T. Lelièvre, D. Le Peutrec, and B. Nectoux. Jump markov models and transition state theory: the quasi-stationary distribution approach. Faraday Discussions, 195:469-495, 2017.

[14] G. Di Gesù, T. Lelièvre, D. Le Peutrec, and B. Nectoux. The exit from a metastable state: concentration of the exit point distribution on the low energy saddle points, part 1. Journal de Mathématiques Pures et Appliquées, 2019. In press: https://doi.org/10.1016/j.matpur.2019.06.003.

[15] L.C. Evans. Partial differential equations, volume 19 of Graduate Studies in Mathematics. American Mathematical Society, Providence, RI, second edition, 2010.

[16] M.I. Freidlin and A.D. Wentzell. Some problems concerning stability under small random perturbations. Theory of probability and its applications, 17(2):269-283, 1973.

[17] M.I. Freidlin and A.D. Wentzell. Random Perturbations of Dynamical Systems. Springer-Verlag, 2012.

[18] A. Galves, E. Olivieri, and M.E. Vares. Metastability for a class of dynamical systems subject to small random perturbations. The Annals of Probability, 1288-1305, 1987.

[19] D. Gilbarg and N. S. Trudinger. Elliptic partial differential equations of second order. Classics in Mathematics. Springer-Verlag, Berlin, 2001. Reprint of the 1998 edition.

[20] P. Hänggi, P. Talkner, and M. Borkovec. Reaction-rate theory: fifty years after kramers. Reviews of modern physics, 62(2):251, 1990.

[21] B. Helffer. Spectral theory and its applications, volume 139. Cambridge University Press, 2013.

[22] F. Hérau, M. Hitrik, and J. Sjöstrand. Tunnel effect and symmetries for Kramers-Fokker-Planck type operators. J. Inst. Math. Jussieu, 10(3):567-634, 2011.

[23] H. Ishii and P.E. Souganidis. Metastability for parabolic equations with drift: part 1. Indiana University Mathematics Journal, 64:875-913, 2015.

[24] H. Ishii and P.E. Souganidis. Metastability for parabolic equations with drift: Part II. the quasilinear case. Indiana University Mathematics Journal, 66(1):315-360, 2017.

[25] H.A. Kramers. Brownian motion in a field of force and the diffusion model of chemical reactions. Physica, 7(4):284-304, 1940.

[26] C. Landim, M. Mariani, and I. Seo. Dirichlet's and Thomson's principles for non-selfadjoint elliptic operators with application to non-reversible metastable diffusion processes. Arch. Rational Mech. Anal., 231(2):887938, 2019.

[27] C. Le Bris, T. Lelièvre, M. Luskin, and D. Perez. A mathematical formalization of the parallel replica dynamics. Monte Carlo Methods and Applications, 18(2):119-146, 2012.

[28] D. Le Peutrec and L. Michel. Sharp spectral asymptotics for non-reversible metastable diffusion processes. ArXiv version : 190\%.09166, 2019.

[29] D. Le Peutrec and B. Nectoux. Repartition of the quasi-stationary distribution and first exit point density for a double-well potential. SIAM J. Math. Anal., 52(1):581-604, 2020.

[30] D. Le Peutrec and B. Nectoux. Small eigenvalues of the Witten laplacian with dirichlet boundary conditions: the case with critical points on the boundary. Analysis \& PDE, to appear, 2020.

[31] T. Lelièvre. Accelerated dynamics: Mathematical foundations and algorithmic improvements. The European Physical Journal Special Topics, 224(12):2429-2444, 2015.

[32] T. Lelièvre, D. Le Peutrec, and B. Nectoux. The exit from a metastable state: concentration of the exit point distribution on the low energy saddle points II, 2019. In preparation.

[33] T. Lelièvre and F. Nier. Low temperature asymptotics for quasistationary distributions in a bounded domain. Anal. PDE, 8(3):561-628, 2015.

[34] T. Lelièvre and G. Stoltz. Partial differential equations and stochastic methods in molecular dynamics. Acta Numerica, 25:681-880, 2016.

[35] R.S. Maier and D.L. Stein. Limiting exit location distributions in the stochastic exit problem. SIAM Journal on Applied Mathematics, 57(3):752-790, 1997. 
[36] F. Martinelli, E. Olivieri, and E. Scoppola. Small random perturbations of finite-and infinite-dimensional dynamical systems: unpredictability of exit times. Journal of Statistical Physics, 55(3-4):477-504, 1989.

[37] P. Mathieu. Spectra, exit times and long time asymptotics in the zero-white-noise limit. Stochastics: An International Journal of Probability and Stochastic Processes, 55(1-2):1-20, 1995.

[38] B.J. Matkowsky and Z. Schuss. The exit problem: a new approach to diffusion across potential barriers. SIAM Journal on Applied Mathematics, 36(3):604-623, 1979.

[39] B.J. Matkowsky and Z. Schuss. Eigenvalues of the Fokker-Planck operator and the approach to equilibrium for diffusions in potential fields. SIAM Journal on Applied Mathematics, 40(2):242-254, 1981.

[40] B.J. Matkowsky, Z. Schuss, and E. Ben-Jacob. A singular perturbation approach to kramers diffusion problem. SIAM Journal on Applied Mathematics, 42(4):835-849, 1982.

[41] L. Miclo. Comportement de spectres d'opérateurs de Schrödinger à basse température. Bulletin des sciences mathématiques, 119(6):529-554, 1995.

[42] B. Nectoux. Analyse spectrale et analyse semi-classique pour la métastabilité en dynamique moléculaire. $\mathrm{PhD}$ thesis, Université Paris Est, 2017.

[43] B. Nectoux. Sharp estimate of the mean exit time of a bounded domain in the zero white noise limit. Markov Processes Relat. Fields, 26(3):403-422, 2020.

[44] L. Nirenberg. On elliptic partial differential equations. In Il principio di minimo e sue applicazioni alle equazioni funzionali, pages 1-48. Springer, 2011.

[45] B. Perthame. Perturbed dynamical systems with an attracting singularity and weak viscosity limits in hamilton-jacobi equations. Transactions of the American Mathematical Society, 317(2):723-748, 1990.

[46] Z. Schuss. Theory and applications of stochastic processes: an analytical approach, volume 170. Springer Science \& Business Media, 2009.

[47] G. Schwarz. Hodge decomposition - a method for solving boundary value problems, volume 1607 of Lecture Notes in Mathematics. Springer-Verlag, Berlin, 1995.

[48] M.R. Sorensen and A.F. Voter. Temperature-accelerated dynamics for simulation of infrequent events. J. Chem. Phys., 112(21):9599-9606, 2000.

[49] M. Sugiura. Exponential asymptotics in the small parameter exit problem. Nagoya Mathematical Journal, 144:137-154, 1996

[50] M. Sugiura. Asymptotic behaviors on the small parameter exit problems and the singularly perturbation problems. Ryukyu Math. J, 14:79-118, 2001.

[51] A.F. Voter. A method for accelerating the molecular dynamics simulation of infrequent events. J. Chem. Phys., 106(11):4665-4677, 1997.

[52] A.F. Voter. Radiation Effects in Solids, chapter Introduction to the Kinetic Monte Carlo Method. Springer, NATO Publishing Unit, 2005. 